\title{
SCLEROSTIN AND WNT-5A GINGIVAL PROTEIN LEVELS IN CHRONIC PERIODONTITIS AND HEALTH
}

\author{
A THESIS SUBMITTED TO THE FACULTY OF THE GRADUATE \\ SCHOOL OF THE UNIVERSITY OF MINNESOTA \\ BY
} GEORGIOS CHATZOPOULOS DDS

IN PARTIAL FULFILLMENT OF THE REQUIERMENTS FOR THE DEGREE OF MASTER OF SCIENCE

LARRY F. WOLFF DDS, MS, PhD

KIM C. MANSKY PhD

May 2018 
GEORGIOS CHATZOPOULOS COPYRIGHT 2018 


\section{ACKNOWLEDGEMENTS}

I would like to acknowledge my major advisor Dr. Larry F. Wolff for his continuous guidance and support throughout this project and my residency. I wish to express my deepest gratitude for his dedication, patience and engagement that have contributed significantly to my progress. I will be forever grateful for his help.

Dr. Kim Mansky for her valuable directions, guidance and support throughout the study. Thank you for the valuable comments and the guidance with the laboratory component of this research project. I wish to extend my appreciation to all PhD candidates and post-doctoral fellows at Dr. Mansky's lab.

I would also like to thank Dr. Massimo Costalonga for providing me with food for thought. His useful comments and suggestions made this project better. His expertise greatly contributed to this project.

Mr. Scott Lunos for the statistical analysis and advice.

Dr. Weihua Guan from the School of Public Health for serving on my examining committee for my thesis.

Dr. James Hinrichs for giving me the opportunity to be at the University of Minnesota and become a periodontist.

Mrs. Josette Fontana and Mrs. Megan Hughes for the administrative support.

My co-residents and dental assistants in the Graduate Periodontology clinic for their help. 
The Erwin Schaffer Chair in Periodontics, University of Minnesota Graduate School, Minneapolis, Minnesota for the financial support of this project. 


\section{DEDICATION}

I would like to dedicate this Master Thesis to my family and friends for their support, love, patience and encouragement.

To my parents, Sokratis and Anastasia, and my brother llias who supported my dream to attend the Advanced Education program in Periodontology at the University of Minnesota, School of Dentistry. Their unconditional and endless love and encouragement were of paramount importance and contributed significantly to achieve all my goals. They taught me to work hard for the things that I want to achieve.

To Villy, who has constantly been by my side and supported me for the last seven years. Thank you for your patience and understanding.

Finally, I would like to thank all my friends who have been there for me during the good times and challenges of the residency. 


\section{ABSTRACT}

Background: Wnt signaling is fundamental to a myriad of biological processes including embryogenesis, organogenesis and tumorigenesis. It drives the expression of important genes for the proliferation, differentiation, maturation and activity of osteoblast-lineage cells. Pathways associated with Wnt signaling are regulated by their physiological antagonists such as sclerostin (SOST) as well as Wnt-5a that play important roles in the pathophysiology of several inflammatory conditions.

Objectives: To compare the levels of SOST, Wnt-5a and TNF- $\alpha$ between chronic periodontitis and periodontally healthy sites as well as to determine the correlation between clinical periodontal parameters and protein levels.

Material and methods: In a cross-sectional assessment, 25 chronic periodontitis cases and 25 periodontally healthy controls were measured for probing pocket depth (PPD), clinical attachment level (CAL), bleeding on probing (BOP), plaque index $(\mathrm{PI})$ and percentage of radiographic bone loss. Gingival crevicular fluid (GCF) was collected cross sectionally from one periodontitis (deepest pocket) and one healthy (shallowest pocket) site in each case, and from one randomly selected site in each control subject. The protein levels of SOST, Wnt-5a and TNF- $\alpha$ in GCF were measured by ELISA. The Shapiro-Wilk test was utilized to assess the normality of the distribution and non-parametric comparisons were performed.

Results: Protein levels of SOST and Wnt-5a were similar between healthy and chronic periodontitis sites as well as the SOST levels between periodontally affected and healthy sites in the chronic periodontitis group $(p>0.05)$. Periodontally involved sites exhibited statistically significantly higher total Wnt-5a levels than the healthy sites in the periodontitis cases $(p=0.017)$. When the data 
were expressed as concentration, significant differences were found among the study groups ( $p$-values range from $<0.001$ to 0.003 ). Significant positive correlations were found between SOST and 1) full-mouth PPD ( $p=0.044)$, CAL ( $p$ $=0.010)$, BOP $(p=0.035) ; 2)$ PPD of the sampled sites $(p=0.037)$; and 3$)$ sites with PPD 4-6 mm $(p=0.018)$. A trend of a negative correlation was found between SOST and sites with a CAL $0-2 \mathrm{~mm}(p=0.052)$. Among all included subjects, males showed a statistically significantly higher Wnt-5a levels than females $(p=$ $0.031)$.

Conclusions: Within the limitations of this cross-sectional study, sites with chronic periodontitis exhibited significantly elevated levels of Wnt-5a when compared to periodontally healthy sites in chronic periodontitis patients. No statistically significant association was found with SOST and TNF-a levels. 
Data from this research project have been presented as oral presentations in the following conferences:

- Midwest Society of Periodontology annual meeting, Chicago, IL. 2018 Graduate Student Research Forum Chatzopoulos G, Mansky K, Costalonga M, Wolff L.

Title: "SOST and Wnt5a gingival protein levels in healthy, gingivitis and periodontitis sites."

Received the $2^{\text {nd }}$ prize in the 2018 Graduate Student Research Forum competition

- 47th Annual Meeting and Exhibition of the American Association for Dental Research (AADR), Fort Lauderdale, FL.

Chatzopoulos G, Mansky K, Costalonga M, Lunos S, Wolff L.

Title: "Gingival Crevicular Fluid Levels of SOST and Wnt5a in Chronic Periodontitis." 


\section{TABLE OF CONTENTS}

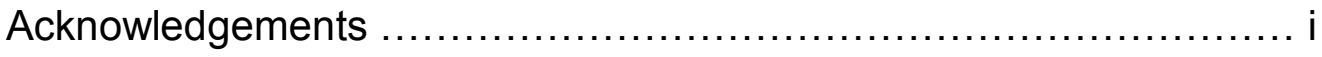

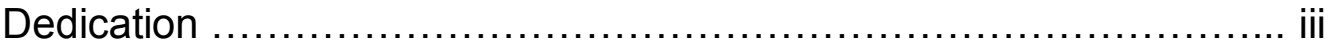

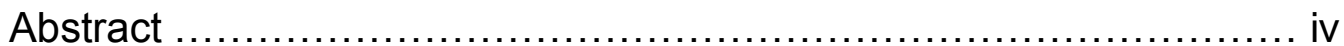

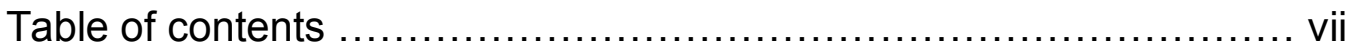

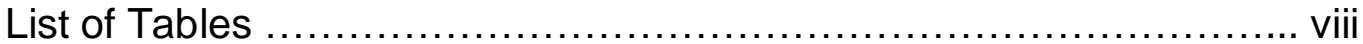

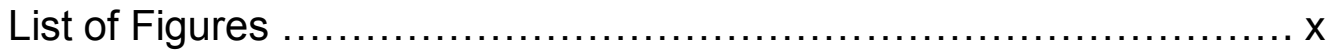

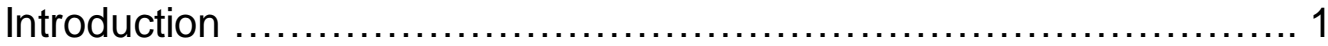

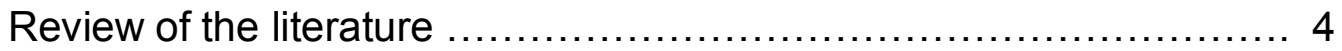

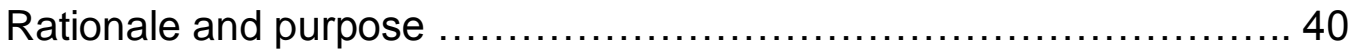

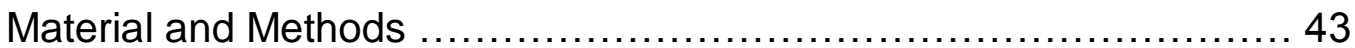

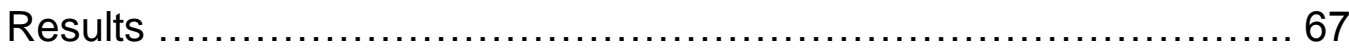

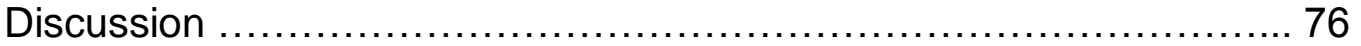

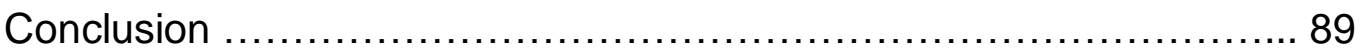

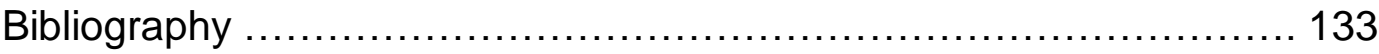




\section{LIST OF TABLES}

Table

Page

Table 1: Demographic characteristics of the total population as well as the 91 periodontitis and healthy groups.

Table 2: Periodontal status of the total population as well as the periodontitis and healthy group.

Table 3: Probing pocket depth and clinical attachment level of sampled sites in healthy and periodontitis group.

Table 4: Bleeding on probing of sampled sites in healthy and periodontitis group.

Table 5: Protein levels and concentrations of SOST, Wnt-5a and TNF- $\alpha$ of periodontitis and healthy patients.

Table 6: Protein levels and concentrations of SOST, Wnt-5a and TNF- $\alpha$ of diseased sites and healthy sites in periodontitis patients

Table 7: Spearman's rank correlations (rho) between protein total levels of SOST, Wnt-5a and TNF- $\alpha$ with the periodontal clinical and age parameters.

Table 8: Spearman's rank correlation (rho) between concentrations of SOST, Wnt-5a and TNF- $\alpha$ with the periodontal clinical and age parameters.

Table 9: Protein total levels of SOST, Wnt-5a and TNF- $\alpha$ in sites with presence and absence of bleeding on probing

Table 10: Protein levels and concentrations of SOST, Wnt-5a and TNF- $\alpha$ with respect to gender 
Table 11: Protein total levels of SOST, Wnt-5a and TNF- $\alpha$ of periodontitis and healthy groups using "strict" criteria for diagnosing periodontal disease and health 


\section{LIST OF FIGURES}

\begin{tabular}{|c|c|}
\hline Figure & Page \\
\hline Figure 1: Bone remodeling cycle & 102 \\
\hline Figure 2: Effect of Wnt signaling pathway on bone mass regulation & 103 \\
\hline Figure 3: Interaction between Wnt $\beta$-catenin signaling and bone cells & 104 \\
\hline Figure 4: Overview of the the Wnt signaling & 105 \\
\hline $\begin{array}{l}\text { Figure 5: Regulation of secretion of the WNT inhibitor, SOST, from } \\
\text { osteocytes inactivates the } \mathrm{Wnt} / \beta \text {-catenin signaling }\end{array}$ & 106 \\
\hline $\begin{array}{l}\text { Figure 6: A schematic diagram showing the role of SOST in Wnt/ß- } \\
\text { catenin and RANKL/RANK. }\end{array}$ & 107 \\
\hline $\begin{array}{l}\text { Figure 7: Wnt-5a can both activate and inhibit the canonical Wnt } \\
\text { signaling depending on the receptor context }\end{array}$ & 108 \\
\hline $\begin{array}{l}\text { Figure 8: Distribution of the total amount of GCF SOST in the study } \\
\text { groups. Dark lines show medians. }\end{array}$ & 109 \\
\hline $\begin{array}{l}\text { Figure 9: Distribution of the total amount of GCF Wnt-5a in the study } \\
\text { groups. Dark lines show medians. }\end{array}$ & 110 \\
\hline $\begin{array}{l}\text { Figure 10: Distribution of the total amount of GCF TNF- } \alpha \text { in the study } \\
\text { groups. Dark lines show medians. }\end{array}$ & 111 \\
\hline $\begin{array}{l}\text { Figure 11a: Distribution of the concentration of GCF SOST in the study } \\
\text { groups. Dark lines show medians. }\end{array}$ & 112 \\
\hline
\end{tabular}


Figure 11b: Distribution of the concentration of GCF SOST in the study groups after removing two observational points (sampled sites) that are distant from the other observations. Dark lines show medians.

Figure 12: Distribution of the concentration of GCF Wnt-5a in the study groups. Dark lines show medians.

Figure 13: Distribution of the concentrations of GCF TNF- $\alpha$ in the study 115 groups. Dark lines show medians.

Figure 14: Distribution of the total amounts of GCF SOST of the 116 diseased and healthy sites in periodontitis patients. Dark lines show medians.

Figure 15: Distribution of the total amounts of GCF Wnt-5a of the 117 diseased and healthy sites in periodontitis patients. Dark lines show medians.

Figure 16: Distribution of the total amounts of GCF TNF- $\alpha$ of the 118 diseased and healthy sites in periodontitis patients. Dark lines show medians.

Figure 17a: Distribution of the concentrations of GCF SOST of the diseased and healthy sites in periodontitis patients. Dark lines show medians.

Figure 17b: Distribution of the concentrations of GCF SOST of the diseased and healthy sites in periodontitis patients after removing an observational point (sampled site) that is distant from the other observations. Dark lines show medians. 
Figure 18a: Distribution of the concentrations of GCF Wnt-5a of the diseased and healthy sites in periodontitis patients. Dark lines show medians.

Figure 18b: Distribution of the concentrations of GCF Wnt-5a of the diseased and healthy sites in periodontitis patients after removing an observational point (sampled site) that is distant from the other observations. Dark lines show medians.

Figure 19: Distribution of the concentrations of GCF TNF- $\alpha$ of the diseased and healthy sites in periodontitis patients. Dark lines show medians.

Figure 20: ROC analysis of total amounts of GCF SOST to diagnose 124 chronic periodontitis.

Figure 21: ROC analysis of total amounts of GCF Wnt-5a to diagnose chronic periodontitis.

Figure 22: ROC analysis of total amounts of GCF TNF- $\alpha$ to diagnose chronic periodontitis.

Figure 23: Distribution of the total amounts of GCF SOST of the diseased and healthy sites in the strictly diagnosed periodontitis and healthy patients. Dark lines show medians.

Figure 24: Distribution of the total amounts of GCF Wnt-5a of the diseased and healthy sites in the strictly diagnosed periodontitis and healthy patients. Dark lines show medians. 
Figure 25: Distribution of the total amounts of GCF TNF- $\alpha$ of the

diseased and healthy sites in the strictly diagnosed periodontitis and healthy patients. Dark lines show medians.

Figure 26: ROC analysis of total amounts of GCF SOST to diagnose generalized chronic periodontitis.

Figure 27: ROC analysis of total amounts of GCF Wnt-5a to diagnose generalized chronic periodontitis.

Figure 28: ROC analysis of total amounts of GCF TNF- $\alpha$ to diagnose generalized chronic periodontitis. 


\section{INTRODUCTION}

Periodontal disease is a chronic inflammatory disease with multifactorial etiology. The onset and progression of the disease are initiated by microorganisms and results from the interaction between periodontal pathogens and the host immune response. Periodontal disease is characterized by destruction of the tooth supporting bone that leads to tooth loss if left untreated (Offenbacher 1996). Periodontitis and tooth loss may have a significant negative impact on oral health-related quality of life and lack of teeth causes functional and psychological problems (Al-Harthi et al. 2013; Nordenram et al. 2013). The high prevalence of periodontal diseases affects nearly half of the adults in the United States with $8.5 \%$ of these individuals having been diagnosed with severe periodontal disease (Eke et al. 2012). The economic burden associated with the management of periodontitis underscore the importance of implementing new diagnostic and therapeutic interventions (Eke et al. 2012).

Bone is a dynamic and active tissue that in healthy adults is characterized by a balance between resorption mediated by osteoclasts and formation mediated by osteoblasts. Osteoblasts, in addition to their role in bone formation, mediate bone resorption via the regulation of osteoclasts (Kobayashi et al. 2003). Osteoclasts are responsible for bone resorption and both osteoclasts and osteoblasts are required for growth and remodeling. The regulation of bone metabolism is a complex process of different signal transduction pathways and depends on several local and systemic factors including cytokines, chemokines, hormones and biochemical stimulation (Phan et al. 2004, Crockett et al. 
2011, Hofbauer and Heufelder 2001, Khosla 2001). Understanding the coupling between bone formation and bone resorption is crucial for better understanding and treating periodontal disease.

Wnt signaling is fundamental to a myriad of biological processes including embryogenesis, organogenesis and tumorigenesis. It drives the expression of important genes for the proliferation, differentiation, maturation and activity of osteoblast-lineage cells (Krishnan et al. 2006). Proteins associated with the Wnt signaling pathways regulate osteoblast differentiation. Wnt signaling also modulates osteoclast differentiation and activity, promotes commitment and differentiation of mesenchymal stem cells as well as suppresses chondrogenesis and adipogenesis leading to stimulation of osteoblasts (Rodda and McMahon 2006). Pathways associated with Wnt signaling are regulated by their physiological antagonists such as sclerostin (SOST) as well as Wnt-5a that play important roles in the pathophysiology of several inflammatory conditions.

Traditional clinical criteria are insufficient for determining sites of active disease or for measuring the degree of susceptibility to further disease progression. A diagnostic tool may provide information to aid differential diagnosis, screening, presence, location, severity or staging and prognosis of a disease. Diagnostics based on oral fluids such as saliva and gingival crevicular fluid (GCF) have attracted scientific interest in periodontal research as well as in a number of systemic diseases including among others cardiovascular diseases and cancer (Taba et al. 2005, Rathnayake et al. 2013). GCF is a site-specific sampling method and less biased by any other oral pathological conditions 
(Uitto et al. 2003). The evaluation of GCF can be used to assess quantitatively the sitespecific inflammatory status of a tooth. Molecules in saliva and GCF correlate with tissue inflammation and bone destruction but due to methodological differences in the existing literature, it is difficult to draw clear conclusions in how to utilize these inflammatory molecules in diagnosis. Therefore, while clinical measures remain reliable indices of disease history, highly specific and sensitive biomarkers are needed for the risk of disease diagnosis and monitoring of periodontal disease activity (Buduneli and Kinane 2011) The initial step in promoting a biomolecule as a biomarker is a cross-sectional assessment in different periodontal health and disease conditions. Cross-sectional studies have been utilized extensively to determine associations, generate hypotheses and ultimately further studies. 


\section{REVIEW OF THE LITERATURE}

\section{Etiology and classification of periodontal diseases}

Plaque-induced periodontal diseases are highly prevalent and can affect up to $90 \%$ of the population worldwide. The manifestations of the disease ranges from gingivitis to periodontitis. Gingivitis affects $50-90 \%$ of adults based on the various definitions and represents a mild and reversible form of periodontal disease (Albandar and Rams 2002). Gingivitis is a reversible inflammation of the gingival tissues without loss of connective tissue attachment and alveolar bone (Armitage 1999). It may also be presented in a reduced but stable periodontium. In a classic study of human experimental gingivitis by Löe and colleagues it was shown that clinical gingivitis developed within 21 days in the absence of oral hygiene which established the etiologic role of plaque in gingival inflammation (Löe et al. 1967). Gingivitis can be reversed if inflammation is resolved and good plaque control is established (van der Weijden and Hioe 2005). However, if biofilm is not controlled, then gingivitis persists and may progress to periodontal disease

(Kornman 2008). In an experimentally induced gingivitis model in young and healthy volunteers, an acute systemic inflammation resulted with enhanced systemic levels of Creactive protein (CRP), interleukin (IL)-6, monocyte chemoattractant protein (MCP)-1 and activated monocytes which consisted of markers and mediators for vascular atherosclerotic disease (Eberhard et al. 2013). Therefore, the presence of gingival inflammation has been associated with systemic effects. 
According to the broadly accepted classification system published in 1999, destructive periodontal diseases are classified into chronic periodontitis, aggressive periodontitis, periodontitis as a manifestation of systemic disease, necrotizing periodontal disease, abscesses of the periodontium, periodontitis associated with endodontic lesions and developmental or acquired deformities and conditions (Armitage 1999). Chronic periodontitis is the most common form of destructive periodontal disease (Papapanou 1996) and is characterized by gingival inflammation with apical migration of the epithelial attachment onto the root surface and loss of connective tissue attachment and alveolar bone (Armitage 1995). It may be either localized or generalized depending on the number of affected sites. Localized periodontitis describes a condition with $30 \%$ or less affected sites, while in its generalized form more than $30 \%$ of the sites are affected (Armitage 1999). Chronic periodontitis is associated with the presence of local etiologic factors such as microbial biofilm and subgingival calculus, gingival inflammation, periodontal pockets and attachment loss that progresses slowly (Brown and Löe 1993, Löe 1986, Papapanou and Wennstrom 1989). Aggressive periodontitis is a type of periodontal disease in which there is rapid destruction of periodontal ligament and alveolar bone which occurs primarily in otherwise systemically healthy young individuals (Armitage 1999).

Different case definitions have been used to describe chronic periodontal disease having a great impact on reporting prevalence, severity and extent of the disease. Clinical examination is commonly performed to determine the presence of the disease as well as its severity and extent (Holtfreter et al. 2015). Severity of periodontitis is commonly categorized based on the amount of clinical attachment loss. Slight or early periodontitis 
exists when attachment loss ranges between 1-2 mm, moderate periodontitis when 3-4 $\mathrm{mm}$ of attachment loss is present and severe periodontitis in the presence of at least 5 $\mathrm{mm}$ of attachment loss (Armitage 2004). Although clinical attachment loss (CAL) is considered an accurate measurement of periodontal disease severity, it may occur even in the absence of disease such as in the case of overhang restorations by plaque accumulation (Page and Eke et al. 2007). A collaboration between the American Academy of Periodontology (AAP) and the Division of Oral Health at the Centers for Disease Control and Prevention (CDC) proposed new definitions for severe and moderate periodontitis (Page and Eke 2007). Patients with two or more interproximal sites with CAL $\geq 4 \mathrm{~mm}$ associated with two or more different teeth or two or more interproximal sites with a probing depth of $\geq 5 \mathrm{~mm}$, not on the same tooth, were considered having moderate periodontitis. Severe periodontitis describes patients with two or more interproximal sites with $\mathrm{CAL} \geq 6 \mathrm{~mm}$, not on the same tooth, and the presence of one or more interproximal sites with a probing depth $\geq 5 \mathrm{~mm}$. In this case definition, interproximal sites were only considered because the disease usually initiates and is most severe at interproximal sites minimizing also the risk of skewing the results due to gingival recessions (Page and Eke 2007).

Criteria for diagnosing periodontal diseases have been confusing throughout the years. The diagnosis of periodontal disease is primarily based on assessment of the destruction of periodontal tissues by the disease and it is not an assessment of the presence of the destructive disease process or activity. Currently, clinical examination is used to assess the presence of disease, while biochemical markers, microbes and histopathology are not 
used in everyday clinical practice. The importance of the various criteria used for defining periodontal disease was demonstrated in a study by Manau et al. that included 14 different periodontitis definitions to examine the relationship between periodontitis and pregnancy (Manau et al. 2008). Six of the 14 definitions showed a statistically significant association of periodontitis with adverse pregnancy events, whereas the other eight did not reach statistical significance. Therefore, it is critical to identify evidence based criteria and standardized methodologies that will lead to an accurate diagnosis of the periodontal diseases.

\section{Risk factors of periodontal disease}

Etiologic factors of the periodontal diseases can be divided into modifiable and nonmodifiable risk factors including age, gender, smoking, diabetes, obesity and metabolic syndrome, osteoporosis, dietary calcium and vitamin D deficiency, stress and genetic factors (Genco and Borgnakke 2013). The prevalence and severity of periodontal disease increase with age which may reflect an individual's cumulative oral history (Genco 1996, Löe et al. 1986). Severe periodontal disease and bone loss are rarely seen in individuals

under 40 years old and the mean annual rate of bone loss differs among different population groups (Hugoson et al. 1992). An annual rate of $0.28 \mathrm{~mm}$ has been reported for individuals at the age of 70 , while $0.07 \mathrm{~mm}$ was found in 25 -year-old individuals (Papapanou and Wennstrom 1989). Males have also been reported to exhibit greater periodontal destruction compared to females, which may be associated with lower levels of oral hygiene among the male population (Slade and Spencer 1995). Although there is 
no clear biological explanation for this association, the difference could also be attributed to sex hormones on the immune system (Haytac et al. 2013). In addition, possible interference by other confounding factors have been reported (Haytac et al. 2013).

Smoking is considered an environmental factor that has been related to a dysbiotic subgingival microbial community which increases the risk for periodontal disease onset (Kumar et al. 2011). A number of epidemiological studies have demonstrated a significant association between smoking/tobacco use and periodontal disease in a variety of different populations suggesting a higher prevalence of severe periodontal disease, increased bone, attachment and tooth loss and gingival recessions than non-smokers (Bergstrom 1989, Bergstrom et al. 2000). Smoking affects all components of the inflammatory response, both the innate and immune host response (Barbour et al. 1997, Johnson and Hill 2004). Neutrophils in smokers have been shown to have decreased chemotaxis, phagocytosis and adherence (Barbour et al. 1997, Johnson and Hill 2004, Kinane and Chestnutt 2000). Smoking is a well-established risk factor for periodontal disease that has been associated with a two- to eight-fold increased risk for periodontal attachment loss and bone loss with heavy smokers exhibiting a much higher risk (odds ratio of 5.6) than light smokers (odds ratio of 2.8) (Bergstrom 2003, Grossi et al. 1995, Torrungruang et al. 2005, Tomar and Asma 2000). There is evidence of a positive relationship between the severity of periodontal disease and the number of pack-years smoked (Grossi et al. 1995).

The strong association of periodontitis with a number of systemic medical conditions has generally been attributed to several factors including systemic distribution of periodontal 
pathogens and local inflammatory mediators. Diabetes is one of the most studied risk factors for periodontal disease (Hajishengallis 2015). A meta-analysis has shown that individuals diagnosed with type 2 diabetes demonstrated a statistically significant higher mean attachment loss of $1 \mathrm{~mm}$ and a greater mean periodontal probing depth of $0.46 \mathrm{~mm}$ compared with non-diabetics (Chavarry et al. 2009). Individuals with type 2 diabetes with poor glycemic control - HbA1c $\geq 7 \%$ (Bandyopadhyay et al. 2010) and $\mathrm{HbA1c} \geq 9 \%$ (Taylor et al. 1998) - demonstrated an increased risk for alveolar bone loss and more severe progression of periodontal disease when compared to those without diabetes and those with controlled diabetes (Bandyopadhyay et al. 2010, Taylor et al. 1998). The higher risk of diabetics for periodontal disease is attributed to the elevated levels of systemic markers of inflammation and the increased inflammatory reaction to bacterial challenge that lead to enhanced periodontal destruction (Brownlee 2005, Dandona et al. 2004).

From a genetic perspective, chronic periodontitis was shown to exhibit a $50 \%$ heritability in a study of twins in which monozygotic twins were more similar than dizygotic twins with respect to all examined clinical measures (Michalowicz et al. 2000). Currently, a number of gene polymorphisms have been associated with periodontal disease with moderate to strong evidence increasing the odds for individual genetic variants up to 50\% (Nibali et al. 2017). Associations between single nucleotide polymorphisms in genes with chronic and aggressive periodontal disease (IL-1 $\beta$, IL-1RN, IL-6, IL-10, CD14, vitamin D receptor, MMP-1 and TLR4) have been reported (Laine 2012). Genome-wide association studies allow a "hypothesis-free" and unbiased analysis of the genome for detecting associations between genetic variants and periodontal disease (Vaithilingam et al. 2014). According to 
the American Academy of Periodontology, "identifying these people with a genetic test before they even show signs of the disease and getting them into early intervention treatment may help them keep their teeth for a lifetime". 


\section{The role of microbiota in periodontal diseases}

It has been reported that the human oral cavity harbors over 700 different bacterial species, of which more than 400 reside within the periodontal pocket (Paster et al. 2006). Development of the dental biofilm is initiated by initial colonizing bacteria and coaggregations between the initial colonizers (Streptococcus species), fusobacteria and late colonizers based on specific cell-cell interactions (Kolenbrander et al. 2010). In periodontitis, the microbial composition generally changes from a gram-positive biofilm to a more complex gram-negative anaerobic biofilm.

A number of studies by Socransky's team used whole genomic DNA probes and checkerboard DNA-DNA hybridization and categorized the composition of plaque from which bacteria were grouped into complexes based on the associations between bacterial species and prevalence in health and disease. The most common species present in supragingival plaque were those of the orange, yellow and blue complexes. Bacteria of the red complex (P. gingivalis, Tannerella forsythia and Treponema denticola) are generally associated with periodontal disease and subgingival plaque (Socransky et al. 1998). The concept of color-coded complexes was adopted worldwide due to its convenience and simplicity. These studies by Socransky and colleagues used culturebased or directed DNA approaches to determine the microbiota in periodontitis and health and showed that microbial counts for 14 species were generally higher in disease than health (Socransky et al. 1998). Such techniques cannot determine the presence of uncultivable species offering limited information on the total microbial diversity. 
Novel molecular techniques including 16S rRNA cloning and sequence as well as $16 \mathrm{~S}$ pyrosequencing provided a much broader picture of differences between periodontal disease and health. A systematic review of the literature included studies that used culture methods, protein electrophoresis techniques and technologies based on nucleic acid detection and showed that seventeen newly identified pathogens were associated with moderate evidence in patients with periodontitis (Perez-Chaparro et al. 2014). However, additional evidence is needed to establish new "true" periodontal pathogens. Phylotypes from the phyla Bacteroidetes, Candidatus Saccharibacteria, Firmicutes, Proetobacteria, Spirochaetes and Synergistetes have been more recently associated with periodontal disease (Perez-Chaparro et al. 2014).

Microbial communities differ between periodontal health and disease. Periodontal disease is associated with a significantly different bacterial community profile, higher levels of gram negative organisms, increased species richness and more uncultivated species than periodontal health (Griffen et al. 2012). Peptostreptococcus, Filifactor, Megashpaera, Desulfobulbus species were significantly associated with periodontal disease, while Streptococcus, Abiotrophia, Gemella, Capnosytophaga and Veillonella species were associated with periodontal health. Differences were more pronounced between healthy and diseased subjects than between deep and shallow pockets, but the microbiome in deep sites demonstrated more genera and species as well as a greater range of diversity values than healthy sites. Microbiome differences have also been reported between race and smoking, while periodontal therapy reduces the microbial richness and diversity in the 
supragingival microbiota (Kumar et al. 2005, Ge et al. 2013, Abusleme et al. 2013, Yamanaka et al. 2012). Therefore, the application of novel molecular techniques that can detect uncultivated species showed that periodontopathogens can be found in periodontal health and that the periodontal microbiota is more heterogeneous and diverse than previously thought (Hajishengallis and Lamont 2012).

It is currently evident that the color-coded complexes require refinement because not only more periodontal pathogens have been shown to play a tremendous role in periodontal disease, but the organisms can also exhibit polymicrobial synergy. Understanding that only the presence of periodontal pathogens cannot result in periodontal disease, led to the polymicrobial synergy and dysbiosis model (PSD model) (Hajishengallis and Lamont 2012). In this model, a diverse microbiota with key pathogens colonizes the periodontal pocket, disrupts the immune surveillance and elevates the virulence of the microbial community leading to a dysbiotic community that disrupts the tissue homeostasis (Hajishengallis and Lamont 2012). The transition from health to disease reflects changes in the abundance of species in the periodontal pocket. Dysbiosis is defined as a shift or imbalance of the relative abundance of species within a microbiome that is associated with disease such as periodontitis. This shift in conjunction with a dysregulated immune response leads to biofilm growth and uncontrolled inflammation. The subgingival dysbiotic environment is rich in immune and inflammatory mediators and provides nutrients for the bacteria to grow. The dysregulation of the host immune system can be a result of immunoregulatory defects such as leukocyte adhesion deficiency or by subversion of the host immune response by oral bacteria of the biofilm (Hajishengallis 2014). P. gingivalis 
has primarily been investigated for its role to modulate the host immune response, but other species also have the potential to modulate the host immune response (Hajishengallis 2014). P. gingivalis has been shown to act as a keystone pathogen due to its ability to remodel a commensal microbial community into a dysbiotic. Keystone pathogen is considered any pathogen that demonstrates a large effect on its environment disproportionately of its abundance. On the other hand, pathobionts are commensal microorganisms that may flourish and promote inflammatory disease under conditions of disrupted homeostasis. Treponema denticola, T. forsythia and Aggregatibacter actinomycetemcomitans are strongly associated with destructive inflammatory responses and subvert the host response by enhancing the survival of bystander species (Hajishengallis 2014).

\section{The role of host immune response in periodontal diseases}

Periodontal disease is defined as a multifactorial inflammatory disease which results from the interaction between periodontal pathogens and the host immune response. Microbial products initiate an inflammatory response leading to local tissue destruction and ultimately tooth loss (Offenbacher 1996). The virulence factors of the subgingival microbes disrupt the immune surveillance and elevate the virulence of the microbial community leading to a dysbiotic community and disruptive tissue homeostasis including periodontal ligament, cementum and alveolar bone (Hajishengallis and Lamont 2012). Although host response is protective against microbial invasion, both hypo- and hyper-responsiveness of a number of pathways can result in enhanced tissue destruction (Preshaw et al. 
2004). Variations in disease onset and progression have been reported in individuals who harbor a similar microbial composition in their dental plaque as well as in patients in whom the periodontal pathogens vary (Baker and Roopenian 2002). Presence of systemic conditions, obesity, smoking, stress and aging characterize a susceptible host that can shift the polymicrobial synergy towards dysbiosis and may influence the progression and severity of periodontal disease (Hajishengallis 2014).

Periodontal bone loss involves "two-way" interactions between the immunoinflammatory and bone-related cells (Graves et al. 2011). Periodontitis is initiated by bacterial plaque in the gingival crevice and occurs in four stages including colonization, invasion, inflammation and irreversible bone loss. More specifically, bacterial accumulation of a biofilm in the gingival sulcus leads to bacterial penetration of the epithelium and connective tissue which stimulates the acquired and innate immune response and ultimately leads to connective tissue attachment and alveolar bone loss (Graves et al. 2011). The interaction between host and bacteria leads to release of a number of cytokines and chemokines which play a pivotal role in periodontal tissue destruction. One of the critical components of the host response to bacteria or their products is a family of receptors, the toll-like receptors (TLRs). TLRs detect and bind microbial components including lipopolysaccharide (LPS), peptidoglycan, bacterial DNA, double-stranded RNA, and lipoprotein, which are called pathogen-associated molecular patterns (PAMPs) (Mahanonda and Pichyangkul 2007). These receptors lead to the activation of several transcription factors such as nuclear factor-kappaB and activator protein 1 (AP-1) through the mitogen-activated protein kinase (MAK) cascade resulting in the subsequent 
production of cytokines and chemokines, many of which directly or indirectly stimulate osteoclast formation (Hayashi et al. 2010, Hans and Hans 2011, Mahanonda and Pichyangkul 2007). Chemokines contribute to periodontal bone loss because they can stimulate recruitment, differentiation and fusion of precursor cells to form osteoclasts or enhance osteoclast survival (Silva et al. 2007).

Pro-inflammatory cytokines such as IL-1 $1 \beta$, IL- 6 and TNF- $\alpha$ link the innate and adaptive immune system by recruiting and activating adaptive immune related cells (Ebersole 2003, Kinane et al. 2011). Recognition of virulent pathogens leads to production of proinflammatory chemokines and cytokines in macrophages, dendritic and epithelial cells; activation and recruitment of complement; emigration of circulating neutrophils; and ultimately stimulation of the adaptive immune responses (Di Benedetto et al. 2013, Kinane et al. 2007, Hajishengallis 2014, Kraus 2010). Neutrophils are present even in a healthy periodontium. Histological evidence has suggested that neutrophils form a barrier between the junctional epithelium and the dental biofilm (Page and Schroeder 1976). Their balanced function is important for the maintenance of periodontal health (Schenkein 2006). Adverse neutrophil counts and genetic neutrophil defects have been associated with severe periodontal disease such as neutropenias, Chediak-Higashi syndrome, Papillon-Lefevre syndrome and granulomatous disease among others (Scott and Krauss 2012). Neutrophils are considered the key protective cell type in periodontal tissues through their phagocytic action, by producing reactive oxygen species and secreting antimicrobial molecules (Scott and Krauss 2012). 
Adaptive immunity is also activated by pro-inflammatory cytokines recruiting circulating lymphocytes and monocytes followed by antigen presenting cells (Kinane et al. 2011). Tcells and B-cells are activated through their interaction with the antigen presenting cells and then they proliferate and migrate to the site of inflammation. A shift from a predominantly $\mathrm{T}$ cell to $\mathrm{B}$ cell lesion in the progression from gingivitis to periodontitis has been reported and the mechanism of the pathogenesis of periodontal disease involves features of both the mucosal $(\lg A)$ and systemic (plasma cells and $\lg M)$ immune systems (Kinane and Lappin 2001). Periodontal lesions are primarily dominated by large numbers of B-cells and plasma cells (Seymour and Greenspan 1979).

Initially, evidence supported that T-cells have a fundamental role in maintaining immune homeostasis and regulate autoimmunity (Gemmell et al. 2007). T-cell mediated adaptive immunity is highly dependent on innate immunity and the CD4+ T-cells were initially divided into two groups based on their pattern of cytokine production: T-helper 1 cytokines that are generally associated with inflammatory bone destruction and T-helper 2 cytokines that have been associated with less bone loss (Murphy and Reiner 2002, Colic et al. 2009). The T-helper $1 / T$-helper 2 paradigm in periodontal disease has been refined since $T$ helper 17 and T regulatory cells were identified (Gaffen and Hajishengallis 2008). T-helper 1/T-helper 2 is no longer valid due discrepancies in studies reported their involvement in diseased tissues, while T-helper 17 and regulatory $\mathrm{T}$ cells have been proposed to demonstrate a counterbalancing mechanism in the local environment with the presence of pro-inflammatory cytokines and TGF- $\beta$ to tip the balance in favor of T-helper 17 or a T regulatory response (Arun et al. 2011). 
T-helper 17 cells play a pivotal role in barrier immunity, are involved in recruitment and activation of neutrophils, participate in immune surveillance and maintenance of barrier integrity as well as regulate antimicrobial peptide production. Increased proportions of Th17 cells and increased IL-17 levels are critical parameters in the pathogenesis of inflammatory diseases such as periodontal disease with a potential effect on osteoclastic mediators (RANKL, TNFa) (Cheng et al. 2014), while T regulatory cells exhibit protective roles against bone resorption through the expression of IL-10 and TGF- $\beta$ (Campbell et al. 2016). Although various cells such as fibroblasts and osteoblasts can express RANKL, Band T-cells are the major source of soluble RANKL revealing the importance of adaptive immunity in periodontal breakdown (Belibasakis and Bostanci 2012). 


\section{Tumor Necrosis Factor}

Tumor Necrosis Factor (TNF) is a pro-inflammatory cytokine that plays an important role in periodontal disease. Its expression in the GCF and periodontal tissues is increased during periodontal breakdown and acts synergistically with the RANK-RANKL system enhancing osteoclast differentiation leading to bone resorption, possibly in response to bacterial LPS (Stashenko et al. 1991, Salvi et al. 1998, Teles et al. 2009, Rogers et al. 2007). TNF may increase osteoclast precursors and increase RANK expression on osteoclast precursors and M-CSF and RANKL expression in stromal cells including osteoblasts and synovial fibroblasts (Yao et al. 2006, Zhang et al. 2008, Teitelbaum and Ross 2006). In primates, an experimental model of periodontitis demonstrated that injection of TNF- $\alpha$ antagonists reduced the number of inflammatory cells in the alveolar bone and the formation of osteoclasts revealing that TNF- $\alpha$ is a significant component of the pathologic process of periodontitis (Assuma et al. 1998). Periodontal destruction was also accelerated following administration of recombinant TNF- $\alpha$ in a rat periodontitis model, while in TNF receptor deficient mice, P. gingivalis-induced osteoclastogenesis was reduced when compared to wild-type controls demonstrating that TNF affects osteoclast formation as part of the host response to bacterial challenge (Gaspersic et al. 2003, Graves et al. 2001). This finding was also confirmed in a study by Garlet et al. which showed that TNF- $\alpha$ receptor has a dual role in A. actinomycetemcomitans-induced experimental periodontitis (Garlet et al. 2007). TNF-a receptor knockout mice developed significantly less inflammation and alveolar bone loss in association with significantly lower levels of IL-1 $\beta$, IFN- $\gamma$ and RANKL in gingival tissues compared to wild-type infected mice 
even in the presence of higher levels of A. actinomycetemcomitans that were associated with lower levels of PMN-related antimicrobial mediator myeloperoxidase. Therefore, these findings provide evidence that TNF- $\alpha$ upregulates the host response to bacteria and stimulates bone resorption in periodontitis (Garlet et al. 2007).

TNF also inhibits bone formation by suppressing osteoblast differentiation. More specifically, TNF inhibits the expression of IGF-1, osterix (OSX), and runt-related transcription factor 2 (Runx2) (Osta et al. 2014, Algate et al. 2016). The inhibition of Runx2 is mediated by the up-regulation of Smurf1 which is a negative regulator of osteoblast differentiation that causes the degradation of Runx2 (Lee et al. 2012). It has also been reported that TNF inhibits bone formation in rheumatoid arthritis by inducing the production of two Wnt signaling antagonists, DKK1 and SOST which control osteoclast and osteoblast differentiation (Goldring et al. 2013, Diarra et al. 2007, Schett 2007). TNF- $\alpha$ may also inhibit the differentiation of mesenchymal stem cells into osteoblasts via the ubiquitin protein ligase Wwp1 in TNF transgenic mice expressing TNF-a (Zhao et al. 2011). However, there are multiple pathways that are generated by inflammatory mediators except for TNF- $\alpha$ that activate osteoclasts and inhibit osteoblast formation. AntiTNF treatment has not shown a significant benefit in bone loss and fracture prevention in inflammatory diseases (Kawai et al. 2012). 


\section{Biology of the bone}

Bone is a living organ, highly dynamic and active mineralized connective tissue that consists of four types of cells: osteoblasts, bone lining cells, osteocytes and osteoclasts (Buckwalter et al. 1996). In bone, specific cells are responsible for the formation, resorption and maintenance of its architecture. Although in early childhood both bone modeling and remodeling occur, bone modeling is the predominant process, while bone remodeling predominates in adulthood (Kobayashi et al. 2003). Osteoblasts are mononucleate bone-forming cells that develop from pluripotent mesenchymal stem cells. They synthetize and secrete type I collagen, which is the major bone matrix protein, and osteoblasts also produce osteoid that mineralizes the newly formed bone. Proteins and signaling pathways such as bone morphogenetic protein (BMP), insulin-like growth factor (IGF), platelet-derived growth factor (PDGF), fibroblast growth factor (FGF), transforming growth factor (TGF) $\beta$ and Wnt signaling pathways are important in regulating osteoblast differentiation and function (Cao and Chen 2005, Hughes et al. 2006, Day et al. 2005, Baron and Kneissel 2013). On the other hand, osteoclasts are the only type of cells that are able to resorb bone. They are large multinucleated cells and derive from mononuclear precursor cells of the monocyte/macrophage lineage (Teitelbaum and Ross 2003). Inflammatory diseases such as rheumatoid arthritis enhances the formation of osteoclasts through the synergistic effect of pro-inflammatory cytokines and RANKL as well as in conjunction with the differentiation of dendritic cells into osteoclasts (Chamoux et al. 2009). 
Bone is consistently being remodeled to maintain a healthy skeleton orchestrated by osteoblasts and osteoclasts. Bone remodeling is a cycle of three phases. Initially, bone resorption is mediated by osteoclasts, then the transition or reversal period follows and finally the bone formation phase which is mediated by osteoblasts (Sims and Gooi 2008). For other authors bone remodeling consists of four phases: activation of osteoclast progenitors via the RANKL/RANK/OPG pathway, bone resorption of the organic and mineral bone components, reversal and bone formation (Wittkowske et al. 2016). Mineralization and osteocytogenesis follows the bone formation. The bone remodeling cycle is shown in Figure 1. Bone remodeling is a physiological process, necessary for fracture healing and skeleton adaptation (Dallas et al. 2013). In normal bone remodeling, a balance between bone resorption and bone formation is ensured, while an imbalance may lead to pathological conditions and development of bone disorders. Excessive resorption by osteoclasts in conjunction with an imbalance bone formation contributes to bone loss (e.g. osteoporosis, periodontal disease etc), whereas the opposite may result in conditions such as osteopetrosis (Khosla et al. 2012, Sobacchi et al. 2013). The regulation of bone metabolism is a complex process of different signal transduction pathways and depends on several local and systemic factors including cytokines, chemokines, hormones and biochemical stimulation (Phan et al. 2004, Crockett et al. 2011, Hofbauer and Heufelder 2001, Khosla 2001). Understanding the coupling between bone formation and bone resorption is crucial for better understanding and treating periodontal disease. 
The primary regulatory mechanism of osteoclasts activity is mediated by members of the TNF receptor superfamily, RANK, RANKL and OPG. Periodontal diseases are a result of imbalances of this system (Teitelbaum and Ross 2000, Lerner 2006). RANKL is expressed at its highest levels in osteoblasts and stromal cells, while fibroblasts and activated T- and B-cells are also capable of expressing it (Yasuda et al. 1998, Takayanagi et al. 2000, Teng et al. 2000, Yun et al. 1998). During an inflammatory response, inflammatory mediators enhance the expression of RANKL. Stimulation of RANK by RANKL leads to differentiation of osteoclast progenitor cells and macrophages into fully differentiated osteoclasts resulting in bone resorption (Lerner 2004, Lerner 2006). Excessive bone resorption is associated with the presence of T-cells that is promoted by RANKL expression and cytokine production (Teng et al. 2000, Hofbauer and Heufelder 2001). On the other hand, OPG is considered as a decoy receptor which suppresses bone resorption and blocks osteoclastogenesis via binding to RANKL and inhibiting the interaction between RANKL and RANK (Simonet et al. 1997). OPG is expressed by human periodontal ligament cells, gingival fibroblasts, epithelial cells and human microvascular endothelial cells (Kanzaki et al. 2002, Sakata et al. 1999). Therefore, the interaction between RANK, RANKL and OPG is important in inflammation-induced bone resorption, such as periodontitis (Liu et al. 2003). The RANKL/OPG ratio is the key pathway regulating the bone resorption and bone formation in a number of bone related disorders including periodontitis, rheumatoid arthritis, osteoporosis and bone cancer (Crotti et al. 2003, Belibasakis and Bostanci 2012). Imbalance in RANKL and OPG leads to alveolar bone destruction. Increased expression of RANKL has been reported in inflamed gingival tissues and in gingival crevicular fluid from periodontitis patients as well as in synovium from rheumatoid arthritis patients (Crotti 
2003, Mogi et al. 2004, Gravallese et al. 2000), while OPG expression is decreased in gingival tissues and gingival crevicular fluid of periodontitis sites compared to healthy sites (Crotti et al. 2003, Mogi et al. 2004). With respect to the RANKL/OPG ratio, increased expression ratio is indicative of the presence of periodontal disease since RANKL and OPG gene expressions are differentially regulated in gingival tissues of different forms of periodontal disease (Bostanci et al. 2007).

\section{Wnt signaling pathways}

Bone metabolism is also regulated by the Wnt signaling pathway. Wnt signaling is fundamental to a myriad biological processes including embryogenesis, organogenesis and tumorigenesis (Kikuchi et al. 2009). Wnt proteins comprise a large family of secreted signaling glycoproteins that regulate cell proliferation, differentiation, apoptosis, survival, migration and polarity in a number of cell types (Kikuchi et al. 2009). It drives the expression of important genes for the proliferation, differentiation, maturation and activity of osteoblast-lineage cells (Krishnan et al. 2006). These Wnt proteins deliver their signs by binding to transmembrane receptor proteins. Proteins associated with the Wnt signaling pathways regulate osteoblast differentiation.

The pivotal role of Wnt signaling in bone formation was displayed in humans with LRP5 mutations (Gong et al. 2001). Loss of function by mutation of LRP5 leads to osteoporosispdeudoglioma syndrome that is associated with decrease bone mass. In mice, inactivation of the LRP5 gene or overexpression of the human LRP5 function gene altered osteoblast 
proliferation, differentiation and apoptosis (Kato et al. 2002, Babij et al. 2003). LRP5 is a Wnt receptor that upon activation leads to stabilization of cytoplasmic $\beta$-catenin. Therefore, inactivation of the LRP5 gene affects the levels of $\beta$-catenin in the cytoplasm. In addition, knockout of $\beta$-catenin in mesenchymal osteoprogenitor cells blocks osteoblast differentiation, expression of mature osteoblast markers and mineralized bone formation, while it promotes chondrocyte differentiation (Day et al. 2005, Hill et al. 2005, Hu et al. 2005, Cui et al. 2011). Disruption of endogenous inhibitors such as DKK1 or SOST increases the ability of Wnt proteins to stabilize $\beta$-catenin and stimulate osteogenesis (Boyden et al. 2002, Li et al. 2005). Wnt signaling pathway impacts bone mass regulation: pathway activation results in an increase in bone mass, while the opposite is observed when the pathway is inhibited (Baron and Kneissel 2013). The effect of Wnt signaling pathway on bone mass regulation is shown in Figure 2.

Wnt signaling also modulates osteoclast differentiation and activity. Wnt/ $\beta$-catenin signaling promotes commitment and differentiation of mesenchymal stem cells as well as suppresses chondrogenesis and adipogenesis leading to stimulation of osteoblasts (Rodda and McMahon 2006). More specifically, Wnt/B-catenin signaling promotes the progression of Osterix1- expressing cells to osteoblasts and prevents the apoptosis of mature osteoblasts via activation of the Src/ERK and PI3K/Akt signaling cascades in both $\beta$-catenin dependent and independent pathways (Almeida et al. 2005). In addition, the signaling pathway stimulates the production and up-regulation of OPG as well as the down-reguation of RANKL that reduces osteoclastogenesis and bone resorption (Glass 
and Karsenty 2006, Spencer 2006). The interaction between Wnt/ $\beta$-catenin signaling and bone cells is demonstrated in Figure 3.

There are two pathways for Wnt signaling; $\beta$-catenin-dependent canonical and $\beta$-cateninindependent non-canonical pathways (Gordon and Nusse 2006, Baron and Kneissel 2013). An overview of the $\mathrm{Wnt} / \beta$-catenin signaling is shown in Figure 4. In the absence

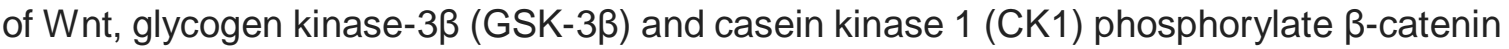
at specific serine/threonine residues in the destruction complex which consists of the tumor supressors axin, the adenomatous polyposis (APC), GSK-3 $\beta$ and CK1. The phosphorylated $\beta$-catenin is ubiquitylated predominantly by the $\mathrm{E} 3$ ligase $\beta$ - $\operatorname{TrCP}$ leading to its degradation by the proteasomes. T cell factor/lymphoid enhancer factor (TCF/LEF) are repressed by Groucho in the nucleus. In the $\beta$-catenin-dependent canonical pathway, Wnt activates multiple intracellular signaling pathways. Wnt proteins bind to a cell-surface receptor which consists of Frizzled and LRP5/6 and inhibit GSK-3 $\beta$ which then promote the accumulation of $\beta$-catenin. The accumulated $\beta$-catenin is translocated to the nucleus, where it binds to the transcription factor TCF/LEF stimulating the transcription of Wnt target genes (Kikuchi et al. 2004, Gordon and Nusse 2006, Logan and Nussa 2004, Baron and Kneissel 2013). In the $\beta$-catenin-independent non-canonical pathways, Wnt proteins bind to the receptor complex of Frizzled and ROR 2 or RYK which recruits dishevelles (DVL) and activate the planar cell polarity (PCP) pathway through the activation of RAC, RHO (small G protein) and Jun kinase (JNK)-dependent signals. The PCP pathway coordinates cell migration and polarity. Another $\beta$-catenin-independent pathway, the $\mathrm{Ca}^{2+}$ pathway, is mediated by Wnt-5a binding to Frizzled and receptor-tyrosine-kinase-like 
orphane receptor $(\mathrm{ROR})$ that increase the intracellular $\mathrm{Ca}^{2+}$ concentration via protein kinase $\mathrm{C}(\mathrm{PKC})$ and calcium/calmodulin-dependent protein kinase (CaMK) II. This pathway inhibits the $\beta$-catenin-dependent pathway and stimulates cell migration (Veeman et al. 2003, Qian et al. 2007, Kühl et al. 2000, Baron and Kneissel 2013).

Mutations of the Wnt pathway have been associated with human skeletal diseases revealing its pivotal role in bone biology and skeletal development (Gong et al. 2011). Mutations of the Wnt canonical pathway are associated with bone mass homeostasis and skeletal patterning. As previously mentioned, LRP5 mutations are associated with osteoporosis pseudoglioma syndrome and with hereditary high bone mass (Gong et al. 2011). Mutations in signaling components such as LRP4, WNT7A, WNT3 and SOST are also associated with congenital limb defects and digit fusion (Baron and Kneissel 2013).

Mutations in genes-regulators of the Wnt pathway such as WNT9B, AXIN2, WTX and SOST have also been associated with bone diseases and congenital craniofacial anomalies including orofacial clefting, tooth agenesis, cranial skeletal dysplasia and cranial sclerosteosis (Chiquet et al. 2008, Lammi et al. 2004, Jenkins et al. 2008, Balemans et al. 2001).

\section{Regulation of the Wnt signaling pathways - Sclerostin (SOST)}

The Wnt pathway in bone is regulated by its physiological antagonists, SOST and Dickkopf-related protein 1 (DKK1) or Wnt ligands such as secreted Frizzed-related proteins and Wnt inhibitory factor $1 / 2$. SOST is a secreted glycoprotein and important 
regulator of Wnt signaling in bone and is primarily expressed by osteocytes (van Bezooijen et al. 2009). Osteocytes influence the balance between bone formation and resorption which is of paramount importance in maintaining normal skeletal health. SOST binds to LRP5/6 on the osteoblast surface and inhibits canonical Wnt signaling by blocking Wnt proteins from binding to the extracellular regions of LRP5 and 6 (Figure 5 and 6). Therefore, SOST decreases osteoblastogenesis and osteoblastic activity (Li et al. 2005, Rossini et al. 2013).

On the other hand, SOST can also activate receptor activator of NF-kappaB ligand (RANKL) and promote osteoclast differentiation and bone resorption (Wijenayaka et al. 2011). Increased SOST activity in bone results in low bone mass, while inhibition leads to increase bone mass and bone strength (Figure 6) (Li et al. 2005, Delgado-Calle et al. 2016, Ominsky et al. 2017). SOST deficiency increases bone mass in mice and humans (Rossini et al. 2013). SOST-neutralizing monoclonal antibodies have been utilized in animal models of osteoporosis, ovariectomized rats and aged male rats showing enhanced bone formation, increased bone mass and bone strength ( $\mathrm{Li}$ et al. 2009, Li et al. 2010). Similar findings were also reported in a ligature-induced periodontitis model in rats in which systemic treatment with anti-sclerostin antibody was delivered twice weekly for up to 6 weeks (Taut et al. 2013). Sclerosteosis, a rare autosomal recessive disorder, and van Buchem disease are related to loss of SOST expression and are characterized by bone overgrown, partial anodontia, delayed tooth eruption and malocclusion (Brunkow et al. 2001, Balemans et al. 2002, Stephen et al. 2001). 
SOST is up-regulated by TNF- $\alpha$ suggesting a potential link between inflammation and osteocyte function via the NK-kappaB pathway (Baek et al. 2014). Induction of SOST by TNF- $\alpha$ in mature osteoblasts is primarily mediated by DKK-1. Blockade of DKK-1 neutralizes the induction of SOST expression by TNF highlighting the cross-talk between TNF, DKK-1 and SOST (Heiland et al. 2010). Wnt signaling pathway was found to be a promising mediator of inflammation that produced bony ankyloses in patients with spondyloarthritis. In this study, a proteoglycan-induced spondylitis (PGISp) mouse model was utilized and 12 weeks after initiation of inflammation, DKK1 and SOST levels were statistically significantly reduced in the spine PGISp in comparison with control mice. The decreased levels of the Wnt antagonists (DKK1 and SOST) in conjunction with the elevated Wnt signaling in spondylitis plays a key role in bone formation (Haynes et al. 2012). In a study that aimed to identify biomarkers for bone metabolism in patients with ankyloses spondylitis, serum levels of SOST were correlated with CRP levels. More specifically, high CRP levels were associated with lower levels of SOST demonstrating a potential connection between inflammation and reduced inhibition of Wnt signaling (Klingberg et al. 2014). Even in a study with the ankyloses spondylitis that included treatment with TNF blockers, persistently elevated CRP levels were also correlated with low SOST levels (Saad et al. 2012).

A cross-sectional study aimed to evaluate the involvement of SOST and DKK1 in human chronic periodontitis (Napimoga et al. 2014). In periodontal tissues of systemically healthy patients with chronic periodontitis, SOST and DKK1 were up-regulated implying a potential involvement in chronic periodontal disease. The levels of SOST were increased in the 
peripheral serum of the periodontal disease patients compared to systemically and periodontally healthy patients. In 2015, a case-control intervention study included 54 individuals and assessed the changes in the levels of SOST, OPG and RANKL in the gingival crevicular fluid (GCF) of periodontitis patients after non-surgical periodontal therapy (Balli et al. 2015). Although the total amounts and concentrations of SOST, OPG and RANKL showed reduction in the periodontitis patients six weeks after the treatment, significant differences were still observed when compared to healthy individuals. This might be attributed to the presence of residual periodontal pockets and active disease.

\section{Regulation of the Wnt signaling pathways - Wnt-5a}

Wnt-5a is an activator of the non-canonical signaling pathway and can bind to multiple receptors including Frizzled 2, 4, 5 and receptor tyrosine kinase-like orphan receptor 2 (ROR2) (Liu et al. 2008). Wnt-5a can also both activate and inhibit the canonical Wnt signaling depending on the receptor context (Figure 7) (Leris et al. 2005). It is the preferred Wnt/PCP activator ligand that binds to Frizzled 2 inhibiting $W n t / \beta$-catenin signaling (Sato et al. 2010, Mikels and Nusse 2006). Similarly, the presence of ROR2 leads to inhibition of the canonical Wnt signaling by promoting $\beta$-catenin degradation through a GSK3-independent pathway which involves the down-regulation of $\beta$-catenininduced gene expression (Topol et al. 2003). The opposite was shown when Wnt-5a binds to Frizzled 4, 5 or LRP5 (He et al. 1997, Mikels and Nusse 2006). Wnt-5a inhibits bone formation by enhancing RANKL-induced osteoclastogenesis (Maeda et al. 2007) and its up-regulated expression may also enhance the expression of inflammatory-related genes 
and intracellular reactive oxygen species through a novel NF-kappaB-dependent regulation (Zhao et al. 2015), which explains the increased expression of Wnt-5a in inflammatory diseases such as atherosclerosis, rheumatoid arthritis, periodontal disease, chronic apical periodontitis, spondyloarthitis as well as in patients with polycystic ovary surgery (Sen et al. 2000, Bhatt and Malgor 2014, Xu et al. 2014, Bougault et al. 2015). In synoviocytes from patients diagnosed with rheumatoid arthritis, the expression of Wnt-5a and Frizzled 5 was significantly up-regulated, while their blockage inhibited synoviocyte activation (Sen et al. 2000, Sen et al. 2001).

Wnt-5a expression depends on TLR signaling and activation of the NF-kappaB in human antigen presenting cells in response to microbial challenge such as $P$. gingivalis (Blumenthal et al. 2006, Nanbara et al. 2012). Wnt-5a up-regulates the production of proinflammatory cytokines such as IL-1 $\beta, \mathrm{IL}-6, \mathrm{IL}-12$ and IL-15 that are dependent on activation of the NF-kappaB pathway and inflammatory mediators in monocytes, macrophages, dendritic cells and microglia. A number of studies have also shown that Wnt-5a induces anti-inflammatory cytokines such as IL-10 in macrophages and dendritic cells and inhibition of the classical TLR4-NF-kappaB signaling pathway (Pereira et al. 2008, Bergenfelz et al. 2012, Oderup et al. 2013). The effects and mechanisms of Wnt-5a on the regulation of inflammation remains unclear.

In periodontitis, the expression of Wnt-5a mRNA is shown to be up-regulated in the gingival tissues of chronic periodontitis patients when compared to healthy gingiva (Nanbara et al. 2012, Maekawa et al. 2017). In a mouse model, Wnt-5a was proposed to 
be an important target for intervention in periodontitis when exogenously added Wnt-5a enhanced LPS-induced inflammation (Maekawa et al. 2017). On the other hand, the same study demonstrated that secreted Frizzed-related proteins (sFRP)-5 was down-regulated by LPS from P. gingivalis and local treatment in mice inhibited inflammation, reduced the numbers of osteoclasts in bone tissues and ultimately inhibited bone loss (Maekawa et al. 2017). An association between WNT-5A gene and severe chronic periodontal disease was also reported in a genome-wide association study by Divaris et al. (2013).

\section{Diagnostics in Periodontology}

Diagnosis of periodontitis has traditionally involved the clinical measurement of probing depth, attachment loss, evaluation of clinical signs of inflammation including bleeding, erythema, edema, exudate as well as radiographic evaluation of the alveolar bone. More recently, molecular determinants have been utilized to establish a biologically-based diagnostic method. These molecular techniques have been used to measure disease activity in regards to severity of tissue destruction and/or inflammation (Loos 2005).

Early detection and prediction of risk for periodontal disease have always been a goal in the field of Periodontology. The use of clinical measures to determine periodontal status does not allow for the accurate prediction of periodontal disease progression or risk of development for periodontal disease (Goodson 1986). Diagnostics based on oral fluids have attracted significant scientific interest in periodontal research as well as in the systemic diseases such as cardiovascular diseases and cancer (Taba et al. 2005, 
Rathnayake et al. 2013). Oral fluids including saliva, oral rinses and GCF can be used in a non-invasive and simple manner for diagnostic purposes. Both saliva and oral rinses are washed out oral fluids, while GCF is a site-specific sampling method and less biased by other oral pathological conditions (Uitto et al. 2003).

\section{Gingival crevicular fluid (GCF)}

GCF arises from the gingival plexus of blood vessels in the gingival connective tissue subjacent to epithelium lining the dentoginigval space (Alfano 1974). In individuals with a healthy periodontal status, GCF is a transudate filtrated from periodontal tissues into the periodontal sulcus that is not mixed with saliva until it flows out of the gingival crevice. GCF is formed as a result of an osmotic gradient in the crevice. On the other hand, trauma or bacterial plaque accumulation stimulate GCF that becomes an inflammatory exudate due to injury and a pathological process (Alfano 1974, Alfano et al. 1976, Griffiths et al. 2003). Hence, GCF is considered protective due to the presence of host protective derived substances existing in the GCF including antibodies, cytokines, enzymes and tissue degradation (Griffiths et al. 2003).

The collection of GCF is somewhat more demanding than the collection of other oral fluids and the avoidance of contamination of the GCF with other oral fluids is important (Griffiths et al. 2003). Another characteristic of GCF is its low amounts in the gingival crevice. In gingivitis and periodontally healthy gingiva, the amount of GCF is low and the detection of biomarkers may be difficult. GCF contains inflammatory mediators, enzymes with 
collagenolytic activity, microbial and host cell enzymes, plasma proteins and proteins associated with the composition of the periodontium (Bowers et al. 1989). Collagen breakdown products are also identified in GCF revealing the connective tissue catabolism for both soft and hard tissues (Champagne et al. 2003). Over 65 GCF components have been examined as possible markers of periodontal disease progression (Armitage 2004). These components have been divided into three groups: host derived enzymes and their inhibitors, tissue breakdown products, inflammatory mediators and host response modifiers (Lamster and Ahlo 2007).

GCF volume is correlated with gingival blood flow and varies based on the severity of periodontal inflammation. The fow rates may increase from $3 \mu \mathrm{L} / \mathrm{h}$ in healthy shallow pockets to $44 \mu \mathrm{L} / \mathrm{h}$ in deep periodontal pockets (Goodson 2003). Increase in GCF volume is associated with an increase in the severity of inflammation. More specifically, GCF volume may be 10 -fold higher in sites with moderate periodontal disease $(0.4 \mu \mathrm{L}, 4-5 \mathrm{~mm}$ pockets) and 30-fold higher in sites with severe periodontal disease (1.5 $\mu \mathrm{L}, 6-9 \mathrm{~mm}$ pockets) compared to healthy sites $(0.05 \mu \mathrm{L})$ (Goodson 2003). Although GCF tends to increase as the inflammation becomes more severe, the quantity of GCF was considered a poor indicator of the severity of gingival inflammation (Hancock et al. 1979). Many other factors may increase the production of GCF such as smoking, oral contraceptives and pregnancy, or orthodontic treatment (Ustun and Alptekin 2007, Klinger 1982, Drummond et al. 2012). These other factors may affect the biomarkers concentration assessment and the interpretation of this evaluation may be misleading to a clinician/investigator. Contamination of GCF may occur with blood, saliva and plaque (Griffiths et al. 2003) and 
prevention of the contamination is critical. GCF samples contaminated with blood or saliva should be discarded. Saliva contamination is prevented by air drying the sample site gently. Careful removal of the supragingival plaque is also recommended, because plaque can affect the GCF volume (Griffiths et al. 2003). Other discrepancies may occur due to evaporation and the recovery time following the sample collection.

Collection of GCF has traditionally been accomplished with absorbent filter strip paper. The filter paper strip inserted into the entrance of the crevice until minimum resistance is felt in order to avoid trauma of the soft tissues (Brill 1959). Collection time generally varies from 30-60 seconds, while shorter duration collection time may be used to avoid contamination with blood or to avoid excessive GCF volume. This technique can be used as a noninvasive, simple and minimal trauma method (Griffith et al. 2003). Electronic devices (Periotron) are then used chairside to identify accurately and time efficiently the GCF volume. It measures the electrical capacitance of the wet paper strip and the greater the moisture in the strip the greater the increase in the capacitance in proportion to the volume of the fluid (Tözüm et al. 2004). Although these electronic devices may measure volumes between $0.1-1.0 \mu \mathrm{L}$, the measurements at the lower end of this range may lack significant accuracy (Griffiths et al. 2003). Measurement of GCF volumes from absorbent paper strip can be extremely reproducible and reliable with the same Periotron device, especially for fluid volumes greater than $0.2 \mu \mathrm{L}$. Calibration of each individual Periotron device will enhance volume accuracy (Chapple et al. 1995). 
GCF can be used to assess quantitatively the site-specific inflammatory status. A biological marker is defined as "an indicator that signals events in biological systems or samples, and it is generally taken to be any biochemical, genetic or immunological indicator that can be measured in a biological specimen (Hulka and Wilcosky 1988, Schulte 1989). Five groups have been proposed by Wilson et al. (2007) and Kinane et al. (2011) for biomarkers based on the information that can provide for periodontal diseases.

- Susceptibility: a biomarker that prospectively identifies individuals or sites at increased risk for periodontal disease.

- Diagnostic: a biomarker that identifies the presence of periodontal disease

- Prognostic: a biomarker that identified patients or sites most likely to respond to specific interventions

- Predictive: a biomarker that predicts future progression of disease

- Therapeutic: a biomarker that provides a quantifiable measure of response to periodontal therapy.

\section{Study designs}

Clinical research, according to Ahrens in 1992, is defined as studies on the mechanisms of human disease; studies on the management of disease; in vitro studies on materials of human origin; animal models of human health and disease; the development of new technologies; the assessment of health care delivery; and field surveys (Ahrens 1992). A number of different study designs exists in clinical research that generally may be separated into observational and experimental studies. Types of clinical research differ 
based on whether the investigators assign the exposures or whether they observed standard clinical practice.

In an observational study, the researcher does not assign an exposure, but the exposure is the agent under investigation to determine its relationship with the health outcome. Studies that the researcher assigns the exposure or treatment are considered experimental. The next step in observation studies is the presence or not of a comparison group. Studies without comparison groups are called descriptive, while comparison groups are included in analytical studies. In the case of analytical studies, the temporal direction of the trial needs to be identified. Assessment of exposures and outcomes at one time point describes a cross-sectional study. On the other hand, if the study initiates with an exposure and the outcome is measured in several time points, then the study design is cohort. Cohort studies can be either concurrent or non-concurrent. In the group of analytical studies, a case-control study begins with an outcome and retrospectively investigates for an exposure (Grimes and Schulz 2002).

Experimental studies can be designed with and without a comparison group. An uncontrolled trial of an intervention is an experiment because the investigator will direct the administration of a treatment, but a concurrent control group is not included in the design phase of the study. Uncontrolled trials of an intervention are useful as part of the early phases of drugs and device development. Experimental studies with a comparison group may be divided into two groups based on the presence of randomization: nonrandomized and randomized. Non-randomized controlled trials of an intervention are used 
primarily when it is unethical or unfeasible to use a random treatment allocation process. When an experiment follows a group of participants over time and has both a comparison group and uses a random process for treatment allocation, it is called a randomized clinical trial. Randomization ensures that the groups under investigation are similar at baseline so any future differences between the groups will likely attributed to the intervention. This allows the study to provide strong evidence of cause and effect. Randomized clinical trials are subdivided into large simple trials, pragmatic trials, explanatory trials and phase II/III trials.

Cross-sectional studies provide descriptive data on prevalence of diseases which provide valuable information for planning analytical studies. In this study design, exposures, which are also called risk factors, and disease endpoints are assessed simultaneously. Crosssectional data cannot provide any assessment of incidence rates but provide estimates of disease prevalence, proportions and estimates of measures of association in order to correlate putative etiologic factors with outcome. Therefore, the question addressed by a cross-sectional study is whether there is an association between a particular factor and a disease or other events. Outcome associations identified from cross-sectional studies are used to generate hypothesis and ultimately provide evidence/data to initiate further studies such as longitudinal investigations.

Cross-sectional studies have been utilized extensively as an initial step to determine associations. These studies are relatively inexpensive and can help determine where funding might be directed to provide maximum benefit. They usually are also quicker to 
conduct than case-control or cohort studies since no follow-up is needed. One of the limitations of a cross-sectional study is that a causal relationship cannot be established. This is because confounding factors cannot be controlled by the investigator. Sometimes it is simply unknown which came first, the exposure or the disease. With cross-sectional studies, there is a risk to infer a causal relationship, exposure to disease, when none may exist. In addition, rare conditions cannot efficiently be studied with cross-sectional studies because a very large number of subjects may be required to yield a sufficient number of diseased individuals. Other study designs including case-series and case-control studies which include patients who already have the disease are more useful than cross-sectional assessment. When cross-sectional associations are used with large representative samples that permit a valid generalization, they can provide useful information about the prevalence of risk factors and disease states. 


\section{RATIONALE AND PURPOSE}

Molecular determinants have been utilized to establish a biologically-based diagnostic method and to measure disease activity in regards to severity of tissue destruction and/or inflammation. There is considerable interest in the identification, validation and clinical application of periodontal biomarkers, because traditional periodontal diagnostic parameters including probing depth, attachment level, bleeding on probing and radiographic assessment of alveolar bone level are inherently limited as these clinical parameters are a measure of disease history. The disease status cannot be assessed since clinical attachment loss readings by a periodontal probe and radiographic evaluation of alveolar bone loss measured damage requires a $2-3 \mathrm{~mm}$ change before a site can be identified as having progression. The primary goal for periodontal biomarkers is to allow earlier detection of disease activity or risk of disease progression and sooner therapeutic intervention.

Inflammatory osteoclastogenesis mediated by pro-inflammatory mediators characterizes the central pathologic process of periodontitis. Therefore, identification of bone markers of periodontal disease may provide information about the presence of current disease activity and allow clinicians to recognize highly susceptible patients or site that are at risk for disease progression. Ultimately the goal is to improve the clinical management of periodontal patients and prevent tissue destruction. The initial step in promoting a biomolecule as a biomarker is a cross-sectional assessment of patients/sites with different periodontal conditions. 
Wnt/ $\beta$-catenin signaling promotes differentiation of mesenchymal stem cells and suppresses chondrogenesis and adipogenesis leading to stimulation of osteoblasts. It also reduces osteoclastogenesis and bone resorption via the up-regulation of the osteprotegrin and down-regulation of RANKL. The Wnt/ $\beta$-catenin signaling is associated with inflammatory responses driven by innate and adaptive immunity via the NF-kappaB pathway. Wnt signaling pathways are regulated by their physiological antagonists such as sclerostin (SOST) as well as Wnt-5a that play important roles in the pathophysiology of several conditions such as osteoporosis and arthritis.

The rationale for this study was to assess the involvement of the Wnt signaling pathway in chronic periodontitis and to examine whether SOST and Wnt-5a in the GCF can be used as diagnostic markers of periodontal disease status.

This research addresses the following hypotheses:

1) Sites with chronic periodontitis exhibit elevated protein total amounts and concentrations of SOST, Wnt-5a and TNF- $\alpha$ compared to periodontally healthy sites.

2) There is a positive correlation between clinical parameters including probing depth and clinical attachment loss with the protein total amounts and concentrations of SOST, Wnt$5 a$ and TNF- $\alpha$ in GCF. 
The specific aims of the study are:

1) To compare the protein total amounts and concentrations of SOST, Wnt-5a and TNFa in GCF between chronic periodontitis and periodontally healthy sites.

2) To determine the correlation between clinical parameters including probing depth and clinical attachment loss with the protein total amounts and concentrations of SOST, Wnt$5 a$ and TNF- $\alpha$ in GCF. 


\section{MATERIAL AND METHODS}

\section{Study design}

This study was designed to cross-sectionally compare the concentration and total amounts of GCF constituents (SOST, Wnt-5a, TNF- $\alpha$ ) in periodontal health and disease. Two groups of individuals classified as having chronic periodontitis and periodontal health were selected for the study. Adult patients with chronic periodontitis ( $\geq 2$ inter-proximal sites with a clinical attachment loss of $\geq 4 \mathrm{~mm}$ and $\geq 2$ inter-proximal sites with probing pocket depth of $\geq 5 \mathrm{~mm}$ and radiographic assessment alveolar bone loss of $\geq 40 \%$ ) were invited to participate in the study. A full-mouth series of $x$-rays within the past 12 months was required to assess the diagnosis of chronic periodontitis. Adults with probing pocket depths $\leq 4 \mathrm{~mm}$, clinical attachment loss $\leq 2 \mathrm{~mm}$ and no evidence of radiographic bone loss were characterized as periodontally healthy patients, and they were included as healthy control subjects. A full-mouth series of intraoral radiographs within the last five years was needed to confirm the presence of a healthy periodontium. Demographic characteristics including age, gender, race, smoking status and medical history were also recorded. The purpose and the procedures of the study were completely reviewed for each evaluated subject, and all patients were given a written consent and HIPAA form in accordance with the Declaration of Helsinki. The study was approved by the Institutional Review Board at the University of Minnesota (Study number: 1511M80307). 


\section{Patient recruitment}

The periodontitis group consisted of patients who were seeking periodontal treatment at the University of Minnesota School of Dentistry, while healthy individuals were identified from patients visiting the Graduate Periodontology clinic for treatment unrelated to periodontal disease. Staff as well as dental and hygiene students were also recruited to participate in the study as healthy volunteers. All eligible individuals who participated in this investigation were compensated with $\$ 20$.

\section{Inclusion and exclusion criteria}

During preliminary screening, all participants were examined for potential eligibility for participation in the study based on the following criteria:

1) at least 18 years old,

2) systemically healthy,

3) $\geq 15$ permanent teeth (excluding third molars) are present,

4) absence of systemic medical conditions that could impact the periodontal status or any bone-related diseases (diabetes mellitus, HIV infection, bleeding disorders, immunosuppressive chemotherapy, osteoporosis, ankylosis spondylitis, recent bone fractures, rheumatoid arthritis, chronic endogenous hypercortisolism) that might influence SOST, Wnt-5a and any additional target molecule levels in GCF levels. 
Moreover, individuals were excluded from the study if they exhibited any of the following criteria:

1) need for antibiotic pre-medication,

2) pregnancy or lactation,

3) antibiotic usage in the last 6 months,

4) periodontal treatment in the last year,

5) regular user of NSAIDs,

6) use of glucocorticoids, bisphosphonates or denosumab,

7) periapical lesions,

8) orthodontic appliances,

9) adults lacking the capability to consent.

\section{Clinical examination}

All subjects underwent a periodontal examination including measurement of probing pocket depth-PPD (distance from the deepest point of the periodontal pocket to the gingival margin) and clinical attachment loss-CAL (distance from the deepest point of the periodontal pocket to the cementoenamel junction) as well as the presence of plaque and bleeding on probing at six sites around each tooth using a Michigan probe with Williams markings. Percentage of sites with PPD $=1-3 \mathrm{~mm}$, PPD $=4-6 \mathrm{~mm}$ and PPD $\geq 7 \mathrm{~mm}$ as well as percentage of sites with $C A L=0-2 \mathrm{~mm}, \mathrm{CAL}=3-4 \mathrm{~mm}$ and $\mathrm{CAL} \geq 5 \mathrm{~mm}$ were also recorded. All measurements were performed at the same six sites of each tooth: mesiobuccal, buccal, disto-buccal, mesio-lingual, lingual, and disto-lingual. Both plaque and 
bleeding on probing were recorded as binary variables, either presence or absence. Calibrated examiners performed the clinical periodontal measurements. Calibration trials were conducted to determine intra- and inter-examiner reliability for the measurement of PPD and CAL.

\section{Calibration}

Prior to conducting a research project in periodontology, a calibration trial should be completed to determine examiners' reproducibility level and measurement variation (error). This is crucial especially when different examiners are used for the recruitment of potential patients. The calibration trial consists of repeated measurements on selected patients in order to determine the level of agreement. The importance of a calibration trial is based on the ability to obtain similar measurements among all examiners as well as each examiner separately. The dimension of the periodontal probe tip, the applied forces, the angle of insertion and the root contour influence the depth recorded (Listgarten 1980). For this study, all measurements were carried out using the same type of periodontal probe (Michigan probe with Williams markings) and training was provided to all examiners. Calibration trials were conducted to determine intra- and inter-examiner reliability for the measurement of PPD and CAL.

Inter-examiner reproducibility or agreement or reliability refers to the consistency of measurements among multiple examiners, while intra-examiner reproducibility is used for 
the consistency of an individual examiner. Examiner reproducibility is expressed as a percentage of both exact agreement and agreement within $\pm 1 \mathrm{~mm}$ between repeated measurements. Exact agreement detects the percentage of the time the examiner reports exactly the same measurement after repeated measurements. On the other hand, agreement within $\pm 1 \mathrm{~mm}$ shows the percentage of the time the examiner's repeated measurement is within $\pm 1 \mathrm{~mm}$ of the initial. Polson recommended using a tolerance of \pm 1 $\mathrm{mm}$ to calculate the percentage of reproducibility agreement in clinical periodontal examiner calibration data, while Best and colleagues reported that a change of $2 \mathrm{~mm}$ in attachment loss could be confirmed by a second examiner (Polson 1997, Best et al. 1990). Evaluating reproducibility for plaque and bleeding is difficult in calibration trials because the measurement process requires removal of plaque, while as far as bleeding is concerned, the first examination may affect the bleeding tendency during the second examination (Abbas et al. 1982).

An initial calibration of the principal (GC) investigation (intra-examiner agreement) was performed with ten individuals: five periodontally healthy and five periodontitis patients. One quadrant of each patient was selected for examination and the assessment was repeated at least one week apart. Examination was performed with a manual periodontal probe (Michigan probe with Williams markings) at six sites per tooth (mesio-buccal, buccal, disto-buccal, mesio-lingual, lingual, and disto-lingual). The right maxillary quadrant and the left mandibular quadrant were randomly selected three times for the calibration, while the left maxillary quadrant and the right mandibular quadrant twice. The percentage of perfect agreement was calculated to be $81.4 \%$ for PPD and $79.8 \%$ for CAL. Agreement 
with PPD $\pm 1 \mathrm{~mm}$ was found in $100.0 \%$ of the sites and agreement with $\mathrm{CAL} \pm 2 \mathrm{~mm}$ in $100.0 \%$ as well.

The purpose of the calibration trial was to quantify intra- and inter-examiner reliability of measuring PPD and CAL. Six individuals recruited to serve in the calibration trial and were different than those utilized in the GCF study. They presented with a range of periodontal health and each examiner assessed PPD and CAL using a manual periodontal probe (Michigan probe with Williams markings) at six sites per tooth (mesio-buccal, buccal, distobuccal, mesio-lingual, lingual, and disto-lingual). Each examiner had one quadrant of teeth examined twice and he/she was blind to the previous measurement. Measuring single quadrants on multiple patients is preferred over multiple quadrants on one or two patients due to patient specific factors that may impact examiner's reproducibility (Janssen and Faber 1988). One examiner performed all periodontal measurements for all four quadrants of each patient and served as the gold standard. For the intra-examiner variability, the first measurements were compared to the second measurements to report the percentage of exact agreement, agreement within $\pm 1 \mathrm{~mm}$ for PPD and agreement within $\pm 2 \mathrm{~mm}$ for CAL. For the inter-examiner variability, measurements of each examiner were compared to the measurements served as the gold standard. Similarly with the intra-examiner variability, exact agreement, agreement of PPD within $\pm 1 \mathrm{~mm}$ and agreement of CAL within $\pm 2 \mathrm{~mm}$ were calculated.

The intra-examiner agreement of the principal (GC) investigator in the second calibration trial was $72.4 \%$ for perfect agreement in PPD, $70.5 \%$ perfect agreement in CAL, $100.0 \%$ 
agreement with PPD $\pm 1 \mathrm{~mm}$ and $100.0 \%$ agreement with $\mathrm{CAL} \pm 2 \mathrm{~mm}$. The percentages of perfect agreement among all examiners ranged from $72.4 \%$ to $82.7 \%$ for PPD and from $47.0 \%$ to $74.4 \%$ for $\mathrm{CAL}$. The agreement between examiners with PPD $\pm 1 \mathrm{~mm}$ ranged from $98.8 \%$ to $100.0 \%$, while the agreement with CAL $\pm 2 \mathrm{~mm}$ was between $98.2 \%$ to $100.0 \%$. Inter-examiner variability was tested between each examiners' measurements and the gold standards' measurements. The exact agreement in PPD varied from 46.0\% to $63.0 \%$ and in CAL from $25.6 \%$ to $57.8 \%$. Agreement in PPD within $1 \mathrm{~mm}$ ranged from 92.1\% to $99.4 \%$, whereas agreement in CAL within $2 \mathrm{~mm}$ was determined to be between $92.9 \%$ and $100.0 \%$.

\section{Sample collection}

In each patient, up to four interproximal non-adjacent sites were selected for sampling after reviewing a patients full-mouth periodontal measurements. GCF sampling was performed either before full-mouth periodontal measurements or at least 2 weeks after the initial comprehensive examination. All subjects were positioned in the supine position in a dental chair. Before GCF sample collection, the supragingival plaque was removed from the interproximal surfaces that were planned to be sampled with a sterile hand instrument. A gentle stream of air was then directed parallel to the buccal and lingual surfaces without disturbing the gingival margin for approximately 3-5 seconds with the use of an air syringe. GCF samples were obtained one at a time using sterile paper strips (Periopaper). Paper strips were gently introduced into the gingival crevice until a mild resistance was felt. GCF collection time was 30 seconds for each strip. Samples that were contaminated with blood 
or visible saliva were discarded. Immediately following GCF collection, the paper strip was placed into a previously calibrated electronic volume quantification unit (Periotron 6000) to determine the collected volume. Filter papers were immediately placed in $5.0 \mathrm{~mL}$ vials and kept on ice until the end of study visit. Samples were then transferred and stored at $80^{\circ} \mathrm{C}$ until processing. All samples were collected by a single examiner (GC).

In the chronic periodontitis group, GCF samples were collected from up to four interproximal non-adjacent sites. Diseased sites exhibited PPD $\geq 5 \mathrm{~mm}, \mathrm{CAL} \geq 4 \mathrm{~mm}$ and radiographic evidence of bone loss. Areas with the greatest inflammation, most significant PPD, CAL and radiographic bone loss were preferred for GCF sampling. When included individuals with chronic periodontitis exhibited healthy sites (PPD $\leq 4 \mathrm{~mm}, \mathrm{CAL} \leq 2 \mathrm{~mm}$ and no evidence of radiographic bone loss), up to two interproximal non-adjacent sites were selected for GCF sampling and the healthiest sites were preferred. In the periodontally healthy participants, samples were collected from up to four interproximal non-adjacent randomly selected sites with PPD $\leq 4 \mathrm{~mm}, \mathrm{CAL} \leq 2 \mathrm{~mm}$ and no evidence of radiographic bone loss

\section{Periotron calibration}

The following steps were performed, following the manufacturer's instructions, to prepare a standard curve relating Periotron readings to volume of fluid in microliters. 
1) A blank paper strip was inserted between the Periotron fluid meter sensors and the instrument was set to zero by turning the dial to the left or right. The blank strip was discarded once it was set to zero.

2) Using a microliter syringe, $0.25 \mu \mathrm{L}$ distilled water was carefully delivered to a paper strip and the strip was placed between the Periotron sensors. The Periotron score was recorded.

3) Step 2 was repeated three more times with this volume $(0.25 \mu \mathrm{L})$ and the average score was recorded.

4) Using a microliter syringe, $0.50 \mu \mathrm{L}$ distilled water was carefully delivered to a paper strip and the strip was placed between the Periotron sensors. The Periotron score was recorded.

5) Step 4 was repeated three more times with this volume $(0.50 \mu \mathrm{L})$ and the average score was recorded.

6) Using a microliter syringe, $0.75 \mu \mathrm{L}$ distilled water was carefully delivered to a paper strip and the strip was placed between the Periotron sensors. The Periotron score was recorded.

7) Step 6 was repeated three more times with this volume $(0.75 \mu \mathrm{L})$ and the average score was recorded.

8) Using a microliter syringe, $1.0 \mu \mathrm{L}$ distilled water was carefully delivered to a paper strip and the strip was placed between the Periotron sensors. The Periotron score was recorded.

9) Step 8 was repeated three more times with this volume $(1.0 \mu \mathrm{L})$ and the average score was recorded. 
10) Using a microliter syringe, $1.25 \mu \mathrm{L}$ distilled water was carefully delivered to a paper strip and the strip was placed between the Periotron sensors. The Periotron score was recorded.

11) Step 10 was repeated three more times with this volume $(1.25 \mu \mathrm{L})$ and the average score was recorded.

Once scores were obtained for all known volumes, a standard curve relating the volume of fluid $(\mu \mathrm{l})$ on the "x" axis to the Periotron score on the "y" axis was prepared. A mathematic equation $(y=m x+b)$ that describes the volume of fluid and the Periotron score was determined based on the standard curve. Paper strips with the collected GCF samples from the study participants were placed between the sensors and a Petriotron score was automatically determined. The volume of GCF was determined by interpolation from the standard calibration curve utilizing the above mentioned equation.

\section{Assay methodologies}

GCF samples were analyzed by an enzyme-linked immunosorbent assay ELISA. Before the quantification of SOST, Wnt-5a and TNF- $\alpha$, GCF samples were removed from $-80^{\circ} \mathrm{C}$ and remained in room temperature for 30 minutes. Then, GCF samples were eluted from the paper strips by placing them in $300 \mu \mathrm{L}$ phosphate buffered saline (PBS) (pH 7.4). The tubes were vortexed and homogenized for 1 minute and then centrifuged at 3,000 rpms for 15 minutes at $4^{\circ} \mathrm{C}$. The supernatants were collected. The total amounts of SOST, Wnt5a and TNF- $\alpha$ in the samples were analyzed separately by sandwich ELISA using 
commercially available kits. Procedures were performed according to the instructions of the manufacturers.

\section{SOST}

Initially all reagents were brought to room temperature for at least 30 minutes before use. A human SOST standard was reconstituted with distilled water $(1 \mathrm{~mL})$ producing a stock solution of $20,000 \mathrm{pg} / \mathrm{mL}$. The standard was mixed to ensure complete reconstitution and it was allowed to sit for a minimum 30 minutes with gentle agitation prior to making any dilutions. Then, $900 \mu \mathrm{L}$ of Callibrator Diluent RD5-20 Concentrate (concentrated buffered protein base with preservatives) were pipetted into a $2,000 \mathrm{pg} / \mathrm{mL}$ tube, and $500 \mathrm{pg} / \mathrm{mL}$ were pipetted into 7 other tubes. The stock solution of $20,000 \mathrm{pg} / \mathrm{mL}$ was used to produce a dilution series. More specifically, $100 \mu \mathrm{L}$ of the stock solution was pipetted into the tube with $900 \mu \mathrm{L}$ of Callibrator Diluent RD5-20 Concentrate to produce $2000 \mathrm{pg} / \mathrm{mL}$ of SOST. Then, $500 \mu \mathrm{L}$ were transferred from this tube to the next one with $500 \mathrm{pg} / \mathrm{mL}$ of Callibrator Diluent RD5-20 Concentrate producing a concentration of $1,000 \mathrm{pg} / \mathrm{mL}$ of SOST. Similarly, tubes of $500 \mathrm{pg} / \mathrm{mL}, 250 \mathrm{pg} / \mathrm{mL}, 125 \mathrm{pg} / \mathrm{mL}, 62.5 \mathrm{pg} / \mathrm{mL}, 31.3 \mathrm{pg} / \mathrm{mL}$ and $0 \mathrm{pg} / \mathrm{mL}$ were produced. Each tube was mixed thoroughly before the next transfer. The 2,000 $\mathrm{pg} / \mathrm{mL}$ of SOST standard served as the highest standard, while the last tube with only Callibrator Diluent RD5-20 Concentrate served as the zero standard (0 pg/mL).

A total of $100 \mu \mathrm{L}$ Assay Diluent RD1X (buffered protein base with preservatives) was added to each well in the microplate. Then, $50 \mu \mathrm{L}$ of standard or sample were added per 
well, covered with an adhesive strip and incubated for 2 hours at room temperature on a horizontal orbital microplate shaker. Wash buffer was prepared by adding $20 \mathrm{~mL}$ of Wash Buffer Concentrate (25-fold concentrated solution of buffered surfactant with preservatives) to distilled water to prepare $500 \mathrm{~mL}$ of wash buffer. Each well was aspirated and washed with $400 \mu \mathrm{L}$ of Wash buffer for a total of 4 washes. Human SOST Conjugate $200 \mu \mathrm{L}$ (polyclonal antibody specific for human SOST conjugated to horseradish peroxidase with preservatives) was added to each well, covered with a new adhesive strip and incubated at room temperature on the shaker. After 2 hours of incubation, aspiration and four washes with $400 \mu \mathrm{L}$ Buffer wash were performed. Stabilized hydrogen peroxide was mixed with stabilized chromogen (tetramethylbenzidine) in equal volumes and $200 \mu \mathrm{L}$ of this solution was added to each well for a 30-minute incubation at room temperature protected from light. The assay was terminated with $50 \mu \mathrm{L}$ of $2 \mathrm{~N}$ sulfuric acid added to each well and the optical density was determined immediately using a microplate reader set to $450 \mathrm{~nm}$ with $570 \mathrm{~nm}$ wavelength correction. The minimum detectable limits of SOST, according to the manufacturer, ranged from 0.37 to $3.80 \mathrm{pg} / \mathrm{mL}$ with a mean value of 1.74 $\mathrm{pg} / \mathrm{mL}$.

\section{Wnt-5a}

Initially, all reagents were brought to room temperature for at least 30 minutes before use. The standard was centrifuged at 10,000 rpms for 30 seconds at room temperature. This standard was then reconstituted with $1.0 \mathrm{~mL}$ sample diluent producing a stock solution of $10 \mathrm{ng} / \mathrm{mL}$ and mixed to ensure complete reconstitution. It was allowed to sit for a minimum 
of 15 minutes with gentle agitation prior to making dilutions. Sample diluent, $250 \mu \mathrm{L}$, was then pipetted into 7 tubes and using the stock solution, a 2 -fold dilution series was produced. More specifically, $250 \mu \mathrm{L}$ were transferred from one vial to another, while each tube was thoroughly mixed before the next transfer. The undiluted standard served as the highest standard (10 $\mathrm{ng} / \mathrm{mL}$ ), while the tube with only $250 \mu \mathrm{L}$ sample diluent served as the zero standard $(0 \mathrm{ng} / \mathrm{mL})$.

A total of $100 \mu \mathrm{L}$ standard or sample was added per well and the microplate was covered with adhesive strip and incubated for 2 hours at $37^{\circ} \mathrm{C}$. Biotin-antibody was centrifuged before opening and a 100-fold dilution was performed with Biotin-antibody diluent to produce Biotin-antibody (1x). Following the 2 hours of incubation, $100 \mu \mathrm{L}$ of Biotinantibody (1x) was added to each well, then the microplate was covered with a new adhesive strip and incubated for 1 hour at $37^{\circ} \mathrm{C}$. Wash buffer was prepared by adding 20 $\mathrm{ml}$ of Wash Buffer Concentrate (25x) into distilled water to prepare $500 \mathrm{ml}$ of Wash buffer (1x). Each well was aspirated and washed with $200 \mu \mathrm{L}$ Wash buffer $(1 \mathrm{x})$ using a multichannel pipette for a total of three washes. HRP-avidin was centrifuged before opening and a 100-fold dilution was performed with HRP-avidin diluent to produce HRPavidin (1x). Following the washes, $100 \mu \mathrm{L}$ of HRP-avidin (1x) was added to each well and then the microplate was covered again with a new adhesive strip and incubated for 1 hour at $37^{\circ} \mathrm{C}$. Aspiration and washes with $200 \mu \mathrm{L}$ of the Wash buffer were then made for a total of five times. TMB substrate $90 \mu \mathrm{L}$ was finally added to each well for a final incubation for 30 minutes at $37^{\circ} \mathrm{C}$ protected from light. The assay was terminated with $50 \mu \mathrm{L}$ of $2 \mathrm{~N}$ sulfuric acid added to each well and the optical density was determined immediately using 
a microplate reader set to $450 \mathrm{~nm}$ with a $570 \mathrm{~nm}$ wavelength correction. The detection of Wnt-5a, according to the manufacturer, ranged from 0.156 to $10 \mathrm{ng} / \mathrm{mL}$ with a minimum detectable dose of less than $0.039 \mathrm{ng} / \mathrm{mL}$.

\section{TNF- $\alpha$}

Initially all reagents were brought to room temperature for at least 30 minutes before use. The standard was then reconstituted with $1.0 \mathrm{~mL}$ sample diluent producing a stock solution of $300 \mathrm{pg} / \mathrm{mL}$ and mixed to ensure complete reconstitution. It was allowed to sit for a minimum of 10 minutes with gentle agitation prior to making dilutions. The stock solution was first diluted to $100 \mathrm{pg} / \mathrm{mL}$ and the diluted standard served as the highest standard (100 pg/mL). Sample diluent $500 \mu \mathrm{L}$ was then pipetted into 7 tubes and using the stock solution, a 2-fold dilution series was produced. More specifically, $500 \mu \mathrm{L}$ were transferred from one vial to another, while each tube was thoroughly mixed before the next transfer. The last tube with only $500 \mu \mathrm{L}$ sample diluent served as the zero standard $(0 \mathrm{pg} / \mathrm{mL})$.

A total of $100 \mu \mathrm{L}$ of the standard or sample were added per well and the microplate was covered with adhesive strip and incubated for 1 hour at $37^{\circ} \mathrm{C}$. Detection $\mathrm{A}$ was centrifuged before opening and a 100-fold dilution was performed with Assay Diluent A to produce Detection Reagent A. Following the 1 hour of incubation, $100 \mu \mathrm{L}$ of Detection Reagent $A$ was added to each well, then the microplate was covered with a new adhesive strip and incubated for 1 hour at $37^{\circ} \mathrm{C}$. Wash buffer was prepared by adding $20 \mathrm{ml}$ of Wash Buffer Concentrate (30x) into distilled water to prepare $600 \mathrm{ml}$ of Wash buffer (1x). Each well 
was aspirated and washed with $350 \mu \mathrm{L}$ Wash buffer $(1 \mathrm{x})$ using a multichannel pipette for a total of three washes. Detection B was centrifuged before opening and a 100 -fold dilution was performed with Assay Diluent B to produce Detection Reagent B. Following the washes, $100 \mu \mathrm{L}$ of Detection Reagent B was added to each well and then the microplate was covered again with a new adhesive strip and incubated for 30 minutes at $37^{\circ} \mathrm{C}$. Aspiration and washes with $350 \mu \mathrm{L}$ of the Wash buffer were then made for a total of five times. TMB substrate $90 \mu \mathrm{L}$ was finally added to each well for a final incubation for 20 minutes at $37^{\circ} \mathrm{C}$ protected from light. The assay was terminated with $50 \mu \mathrm{L}$ of $2 \mathrm{~N}$ sulfuric acid added to each well and the optical density was determined immediately using a microplate reader set to $450 \mathrm{~nm}$. The detection of TNF- $\alpha$, according to the manufacturer, ranged from 1.56 to $100 \mathrm{pg} / \mathrm{mL}$ with a minimum detectable dose of less than $0.52 \mathrm{pg} / \mathrm{mL}$.

\section{Analysis of GCF levels and concentration}

The levels of SOST, Wnt-5a and TNF- $\alpha$ in each sample were extrapolated from the known quantities on the standard curve of each ELISA plate based on the dilutions. The results were expressed as the total amount in 30 seconds and the concentration in the GCF sample. Calculation of the concentration data was performed by dividing the amount of each protein by the volume of the sample as determined by the standard curve of the volume of fluid and the Periotron score. Total values of SOST and TNF- $\alpha$ were measured in pg, while total values of Wnt-5a were expressed in ng. Values of concentrations of SOST and TNF- $\alpha$ were presented as $\mathrm{pg} / \mu \mathrm{L}$, whereas Wnt-5a concentration was expressed in $\mathrm{ng} / \mathrm{mL}$. 


\section{Sample size calculation}

The primary outcome variables (GCF levels of SOST and Wnt-5a) were used to decide the sample size calculation and determine the power of the study. Due to lack of available information in the literature surrounding GCF levels of SOST and Wnt-5a in chronic periodontitis and periodontally healthy patients, no sample calculation could be performed. A pilot study was initially conducted including five periodontitis patients and five healthy control individuals in order to determine the sample size of this research. We estimated that a sample size of 22 patients per group would allow for a type II error level of $\beta=0.05$ (95\% power) and a type I error level of $\alpha=0.05$ ( $5 \%$ probability). To account for possible complications with the GCF samples, a total of 25 patients in each group were included.

Sample size determination is an important parameter in planning an epidemiological study. An adequate sample size ensures that the study will show reliable information regardless of the results from the statistical analysis (e.g whether the ultimate data suggest a statistically significant difference or not). Sample size is the number of subjects in a group under investigation. The larger the sample size, the greater the precision and therefore the power to detect an effect of a given size. In contrast, if a very large sample is used, then even small differences from the null hypothesis will be statistically significant, even if these are not clinically relevant.

Power-based sample size calculations relate to hypothesis testing. It depends on the null hypothesis, the significance level, type I and type II errors. The null is that there is no 
difference between the two groups. The statistical analysis of the study will demonstrate either sufficient evidence to reject the null or not enough evidence to reject the null hypothesis. There are four possible outcomes performing a statistical hypothesis that depend on whether the null hypothesis is true or false and whether the examiner decides to reject or retain it. When the null hypothesis is true, the probability of making a type I error is called alpha ( $\alpha$ ). Type I error is the incorrect rejection of the null hypothesis or in other words when the statistical analysis reveals a significant difference when there is not really a difference. It is also called a "false positive" finding. When the null hypothesis is false, the probability of making a type II error is called beta $(\beta)$. Type II error is the incorrect retention of a false null hypothesis, or in other words when the statistical analysis shows non-significant results when there is really a significant effect. This may be attributed to inadequate power and/or sampling error. Type II error is also called "false-negative" finding. The statistical power is the probability of not missing a significant effect due to sampling error when there is an effect.

The general formula for sample size calculation for two groups using a continuous outcome is:

$$
N \text { per group }=\frac{2 \times(Z a+Z \beta)^{2}}{d^{2}}
$$

Where $d$ is the effect size. 
Effect size is defined as the difference between the means for the two groups, divided by an estimate of the standard deviation in the population. The effect side $d$ can be calculated using the following formula:

$$
d=\frac{\overline{x_{1}}-\overline{x_{2}}}{S_{\text {pooled }}}
$$

The estimate of the standard deviation in the population can be calculated from the following formula:

$$
S_{\text {pooled }}=\sqrt{\frac{\left(n_{1}-1\right) s_{1}^{2}+\left(n_{2}-1\right) s_{2}^{2}}{n_{1}+n_{2}-2}}
$$

Therefore, the number needed per group is proportional to the square of the standard deviation and inversely proportional to the square of differences between the two groups (delta). The more variability (standard deviation), the larger the sample that is needed, while the greater the true difference between the groups, the smaller the sample size.

$$
N \text { per group }=\frac{2 \times s_{\text {pooled }}^{2} \times(Z a+Z \beta)^{2}}{\text { delta }^{2}}
$$




\section{Statistical analysis}

Descriptive statistics were used to present the results of the sample's characteristics including mean and standard deviation for age, number of teeth, periodontal parameters and GCF protein levels, while counts and percentages were used for categorical variables such as gender, race and smoking history. The Shapiro-Wilk test was used to determine whether the data were normally distributed. Normal is used to describe a symmetrical, bell-shaped curve, which exhibits the greatest frequency of scores in the middle, close to the mean value. The null hypothesis of this test is that the population is normally distributed. Therefore, if the p-value is less than the significance level, then the null hypothesis is rejected and there is evidence that the data tested are not normally distributed. In this study, the null hypothesis was rejected because $p<0.001$ suggesting violation of the assumption of normality. The Shapiro-Wilk test statistic W is defined as the ratio of two estimates of the variance of the sample:

$$
W=\frac{\sum_{i=1}^{n} a_{i} x_{i}^{2}}{\sum_{i=1}^{n}\left(x_{i}-\bar{x}\right)^{2}}
$$

where

- $\bar{x}=\frac{\sum_{i=1}^{n} x_{i}}{n}$ is the sample mean.

- $\mathrm{x}_{\mathrm{i}}$ is the order statistic, implying that the data sample must be sorted in increasing values $\left(x_{1}, x_{2}, \ldots, x_{n}\right)$

- $\alpha_{i}$ is the Shapiro-Wilk coefficients 
Non-parametric comparisons were therefore performed. A Mann-Whitney U test was used to compare protein total amounts and concentrations between healthy patient sites and diseased sites in periodontitis patients. A Wilcoxon signed-rank test was utilized to compare diseased sites and healthy sites in a subset of periodontitis patients that exhibited both diseased and healthy sites. The Spearman's rank correlation test was used to detect the relationship between GCF total amounts and concentrations of SOST, Wnt5a and TNF- $\alpha$ with the periodontal clinical parameters including PPD (full-mouth and sampled sites), CAL (full-mouth and sampled sites), percentage of sites with PPD = 1-3 $\mathrm{mm}, \mathrm{PPD}=4-6 \mathrm{~mm}, \mathrm{PPD} \geq 7 \mathrm{~mm}, \mathrm{CAL}=0-2 \mathrm{~mm}, \mathrm{CAL}=3-4 \mathrm{~mm}$ and $\mathrm{CAL} \geq 5 \mathrm{~mm}$ as well as the patient's age. Receiver operating characteristic (ROC) curves were constructed for the total amounts of SOST, Wnt-5a and TNF- $\alpha$ of healthy and periodontitis groups. The area under the curve and the $95 \%$ confidence intervals were computed for each protein. All data were analyzed with SPSS v24 (SPSS Inc. IMB, USA) or SAS v9.3 (SAS Institute Inc., Cary, NC, USA). P-values less than 0.05 were considered statistically significant. Graphing was performed with GraphPad Prism software.

\section{Mann-Whitney U test}

The Mann-Whitney $U$ test is used to compare differences between two independent groups when the dependent variable is either ordinal or continuous, but not normally distributed. It is the non-parametric alternative to the independent t-test and tests the null hypothesis that the distributions of both populations are equal. Calculation of the MannWhitney $U$ test can be performed using the following formula: 


$$
U=U_{1}+U_{2}
$$

where

$$
U_{1}=R_{1}-\frac{n_{1}\left(n_{1}+1\right)}{2}
$$

and

$$
U_{2}=R_{2}-\frac{n_{2}\left(n_{2}+1\right)}{2}
$$

$n_{1}$ and $n_{2}$ are the sample sizes for sample 1 and 2

$R_{1}$ and $R_{2}$ are the sum of the ranks in sample 1 and 2 . Each observation is assigned to a rank, i.e. 1 to the smallest observation, 2 to the second smallest observation etc.

Therefore, the following formula is used:

$$
U=n_{1} n_{2}+\frac{n_{2}\left(n_{2}+1\right)}{2}-\sum_{i=n_{1}+1}^{n_{2}} R_{i}
$$

where

$\mathrm{n}_{1}$ : sample size of sample 1

$\mathrm{n}_{2}$ : sample size of sample 2

$\mathrm{R}_{\mathrm{i}}$ : rank of the sample size 


\section{Wilcoxon signed-rank test}

The Wilcoxon signed-rank test is used to compare differences between two related samples, either matched items or repeated measurements of the same sample that are not normally distributed. It is the non-parametric alternative to the paired t-test and tests the null hypothesis that the distributions of two dependent populations are equal. For each item in a sample the test obtains an absolute difference score between two measurements. Calculation of the Wilcoxon signed-rank test can be performed using the following formula for large samples:

$$
Z=\frac{W-\mu_{W}}{\sigma_{W}}
$$

where the test statistic W is obtained as the sum of the positive ranks (the absolute difference between the pair of values):

$$
W=\sum_{i=1}^{n} R_{i}^{(+)}
$$

and

$$
\mu_{W}=\frac{n(n+1)}{4}
$$


and

$$
\sigma_{W}=\sqrt{\frac{n(n+1)(2 n+1)}{24}}
$$

with $\mathrm{n}$ being the sample size, i.e. the number of pairs.

$\mathrm{Z}$ is then tested against the standard normal deviate $\mathrm{Z}_{\text {critical. }}$. The null hypothesis is rejected if $|Z|>Z_{\text {critical. }}$

\section{Spearman's rank correlation test}

The Spearman's rank correlation test is a non-parametric measure of rank correlation, the alternative of the Pearson correlation coefficient. It measures the strength and direction of the monotonic association between two ranked variables. A monotonic relationship is one that either never increases or never decreases as its independent variable increases. It can range from -1 , which shows strong negative correlation, to +1 , which demonstrates strong positive correlation.

Calculation of the Spearman's rank correlation test can be performed using the following formula for data that do not have tied ranks (data that have different values):

$$
\rho=1-\frac{6 \sum d_{i}{ }^{2}}{n\left(n^{2}-1\right)}
$$


where

$d_{i}$ : the difference in paired ranks (difference between the two ranks of each observation)

$\mathrm{n}$ : the number of cases

Calculation of the Spearman's rank correlation test can be performed using the following formula for data with tied ranks (data that have the same value):

$$
\rho=\frac{\sum_{i}\left(x_{i}-\bar{x}\right)\left(y_{i}-\bar{y}\right)}{\sqrt{\sum_{i}\left(x_{i}-\bar{x}\right)^{2}} \sum_{i}\left(y_{i}-\bar{y}\right)^{2}}
$$

where

i: paired score

\section{ROC curve}

The receiver operating characteristic $(\mathrm{ROC})$ curve is a fundamental tool for diagnostic test evaluation. The true positive (sensitivity) is plotted against the false positive rate (1specificity) for all possible cutoff values. Each point represents a value with a specific sensitivity and specificity. The area under the ROC curve is a measure of the accuracy of a diagnostic test. It is a measure of how well a parameter can distinguish between two diagnostic groups (disease and health) in a diagnostic test. The higher the area under the curve $(A \cup C)$ values, the better test performance. The AUC values can range from 0.5 (no diagnostic value) to 1.0 (excellent diagnostic value). P-value tests the null hypothesis that the area under the curve really equals 0.50 . 


\section{RESULTS}

\section{Demographic characteristics}

The demographic characteristics of the total population as well as the periodontitis and healthy groups are shown in Table 1. The total population of the study consisted of 50 patients with a mean age of $43.4 \pm 18.5$ years. Twenty-five individuals diagnosed with chronic periodontal disease were included in the periodontitis group (50\%), while the healthy group consisted of 25 subjects with a healthy periodontium (50\%). A statistically significant difference was detected in regards to the mean age of the participants in the periodontitis and healthy groups $(p<0.001)$. More specifically, patients diagnosed with chronic periodontitis were $57.9 \pm 12.6$ years old, whereas healthy individuals were significantly younger and had a mean age of $28.9 \pm 10.2$ years. No significant differences were observed between the two groups with respect to gender $(p=0.077)$ and race $(p=0.070)$. In total, $18(36.0 \%)$ males and $32(64.0 \%)$ females participated in this crosssectional study and the majority of the individuals were Caucasians (40\%). Most of the participants in both periodontitis and healthy groups were females and Caucasians, while Asians presented only in the healthy group (4\%) and Hispanics only in the periodontitis group (4\%). Smoking status was recorded as never smokers, ex-smokers and current smokers. Smoking history was significantly different $(p<0.001)$ between the two groups with current smokers $(n=9,18 \%)$ and ex-smokers $(n=6,12 \%)$ being only in the periodontitis group, while never smokers were only included in the healthy group $(n=25$, $50 \%$ ). Current smoking was reported by $18 \%$ of the total population and all of them self- 
reported cigarette smoking. The frequency of smoking was reported as $<10$ cigarettes/day by $55.5 \%, 10-20$ cigarettes/day by $44.4 \%$ and $>20$ cigarettes/day by $11.1 \%$. The mean duration of smoking was 30.8 years with a range from 20 to 55 years.

\section{Periodontal status}

The periodontal status of the total population as well as the periodontitis and healthy groups are presented in Table 2. The periodontal status of the periodontitis group was statistically significantly worse than in the healthy group as expressed by the significantly different clinical periodontal measures. More specifically, the periodontitis group exhibited significantly higher mean values of PPD ( $p<0.001)$, CAL $(p<0.001)$, BOP $(p<0.001)$, $\mathrm{PI}(p=0.001)$, missing teeth $(p<0.001), \mathrm{PPD}=4-6 \mathrm{~mm}(p<0.001), \mathrm{PPD} \geq 7 \mathrm{~mm}(p=$ 0.002), $C A L=3-4 \mathrm{~mm}(p<0.001)$ and $C A L \geq 7 \mathrm{~mm}(p<0.001)$ than the healthy group. On the contrary, the healthy group showed statistically significantly higher mean values of PPD $=1-3 \mathrm{~mm}(p<0.001)$ and $C A L=0-2 \mathrm{~mm}(p<0.001)$ than the periodontitis group.

Sampled sites were divided into three groups: diseased sites in periodontitis patients, healthy sites in periodontitis patients and healthy sites in healthy patients. The PPD and CAL of the sampled sites in healthy and periodontitis groups are shown in Table 3 . Diseased sites in periodontitis patients were associated with higher PPD and CAL than healthy sites in periodontitis and healthy patients. The BOP of the sampled sites in healthy and periodontitis groups is presented in Table 4. In general, diseased sites in the periodontitis group exhibited a higher frequency of BOP than healthy sites in periodontitis 
and healthy individuals. Ninety-two percent of the sampled diseased sites showed BOP, while the majority of the healthy sites in periodontitis patients (78.6\%) and the healthy sites in periodontally healthy patients (20.0\%) demonstrated absence of BOP. Healthy sampled sites from the periodontitis and healthy groups exhibited a similar periodontal status.

\section{Protein levels and concentrations of periodontitis and heathy patients}

The protein levels and concentrations of SOST, Wnt-5a and TNF- $\alpha$ of periodontitis and healthy patients are reported in Table 5. The protein levels of SOST, Wnt-5a and TNF- $\alpha$ were similar between the two groups ( $p>0.05$ ). More specifically, the median of SOST was slightly higher in the periodontitis group $(135.5 \mathrm{pg})$ when compared to the healthy group $(117.9 \mathrm{pg})$ without reaching statistically significance $(\mathrm{p}=0.068)$. The median of Wnt5a found to be $1.9 \mathrm{ng}$ for the periodontitis patients which was lower but statistically insignificant $(p=0.854)$ in comparison to the median of the healthy patients $(2.4 \mathrm{pg})$. Similarly, the healthy group showed an insignificantly $(p=0.071)$ higher median value of TNF- $\alpha$ (40.3 pg) when compared to the periodontitis group (27.2 pg). The distributions of the total amounts of GCF SOST, Wnt-5a and TNF- $\alpha$ in the periodontitis and healthy groups are presented in Figures 8, 9 and 10 with no significant differences found between the chronic periodontitis and periodontally healthy groups $(p>0.05)$.

When the data were expressed as concentration, significant differences were found among the study groups $(p<0.05)$. Periodontally healthy individuals showed statistically significantly higher concentration of SOST $(p<0.001)$, Wnt-5a $(p=0.003)$ and TNF- $\alpha(p$ 
$<0.001)$ compared to the periodontitis patients. However, the results obtained based on the concentrations should be interpreted with caution due to the increased variability as expressed by the range and the standard deviation. The distributions of the concentrations of GCF SOST, Wnt-5a and TNF- $\alpha$ in the periodontitis and healthy groups are presented in Figures 11(a, b), 12 and 13.

The protein levels and concentrations of SOST, Wnt-5a and TNF- $\alpha$ at diseased and healthy sites in a subset of the periodontitis patients that exhibited both diseased and healthy sites are reported in Table 6. Significant differences were found among the sampled sites in the periodontitis group in regards to the total protein levels of Wnt-5a. More specifically, the diseased sites exhibited a median Wnt-5a value of $1.6 \mathrm{ng}$ which was statistically significantly higher $(p=0.017)$ than the median Wnt-5a of the healthy sites (1.1 ng). The protein levels of SOST and TNF- $\alpha$ in the GCF of healthy and periodontitis sites were similar (SOST: $135.5 \mathrm{pg}$ at diseased versus $138.8 \mathrm{pg}$ at healthy sites; TNF- $\alpha$ : $21.9 \mathrm{pg}$ at diseased versus $17.7 \mathrm{pg}$ at healthy sites) and the significant level was not reached $(p>0.05)$. Diseased sites showed a slightly higher level of TNF- $\alpha$ than the healthy sites $(p=0.194)$, while the opposite was found for SOST $(p=0.413)$ but no significant differences were found. The distributions of the total amounts of GCF SOST, Wnt-5a and TNF- $\alpha$ of the diseased and healthy sites are shown in Figure 14, 15 and 16.

With respect to concentrations, SOST, Wnt-5a and TNF- $\alpha$ were statistically significantly different between the two examined groups. Similarly with the results from the comparison between periodontitis and healthy patients, healthy sites demonstrated statistically 
significantly higher concentrations of SOST $(p=0.001)$, Wnt-5a $(p=0.001)$ and TNF- $\alpha(p$ $=0.001)$ when compared to diseased sites. Although the level of significance was reached, the variability present in the sampled sites impact the ability to draw conclusions based on these findings. The distributions of the concentrations of GCF SOST, Wnt-5a and TNF- $\alpha$ of the diseased and healthy sites are demonstrated in Figure 17, $18(\mathrm{a}, \mathrm{b})$ and 19.

\section{Correlations}

The Spearman's rank correlations (rho) between protein total levels of SOST, Wnt-5a and TNF- $\alpha$ with the periodontal clinical and age parameters are shown in Table 7. A statistically significant positive correlation was found between the total amounts of SOST with fullmouth PPD $(r=0.29, p=0.044)$, full-mouth CAL $(r=0.36, p=0.010)$, PPD of the sampled sites $(r=0.30, p=0.037)$ and PPD $=4-6 \mathrm{~mm}(r=0.33, p=0.018)$. In addition, a statistically significant negative correlation was detected with the percentage of sites with PPD $=1-3$ $\mathrm{mm}$ and the total amounts of SOST $(r=-0.34, \mathrm{p}=0.017)$. A trend towards negative correlation was observed with $C A L=0-2 \mathrm{~mm}(r=-0.28, p=0.052)$, while a trend towards positive correlation was found with age $(r=0.27, p=0.055)$. The total amounts of Wnt-5a were not statistically significantly associated with any of the examined parameters $(p>$ 0.05). When the protein levels of TNF- $\alpha$ were examined, a statistically significant negative correlation was noted with the PPD of the sampled sites $(r=-0.29, p=0.039)$. A trend towards negative correlation was also observed between the total amount of TNF- $\alpha$ with age $(r=-0.28, p=0.052)$. 
The Spearman's rank correlations (rho) between concentrations of SOST, Wnt-5a and TNF- $\alpha$ with the periodontal clinical and age parameters are shown in Table 8. Statistically significantly correlations were found between the concentrations of SOST, Wnt-5a and TNF- $\alpha$ with all examined parameters. In particular, statistically significant negative correlations were detected between full-mouth PPD, full-mouth CAL, PPD of the sampled sites, CAL of the sampled sites, PPD $=4-6 \mathrm{~mm}, \mathrm{PPD} \geq 7 \mathrm{~mm}, \mathrm{CAL}=3-4 \mathrm{~mm}, \mathrm{CAL} \geq 5$ $\mathrm{mm}$ and age with the concentrations of SOST, Wnt-5a and TNF- $\alpha$ ( $p$-values range from < 0.001 to 0.016$)$. Moreover, statistically significant positive correlations were observed between the healthy periodontal parameters, $\mathrm{PPD}=1-3 \mathrm{~mm}$ and $\mathrm{CAL}=0-2 \mathrm{~mm}$, with the concentrations of SOST, Wnt-5a and TNF- $\alpha$ ( $p$-values range from $<0.001$ to 0.015$)$.

\section{ROC analyses for diagnostic value}

ROC analyses of the total amounts of GCF SOST, Wnt-5a and TNF- $\alpha$ were performed to diagnose chronic periodontitis. ROC analysis of total amounts of GCF SOST to diagnose chronic periodontitis is shown in Figure 20. The area under the ROC curve for GCF SOST was 0.652 , the $95 \%$ confidence interval was 0.490 and 0.814 and the p-value was 0.065 . ROC analysis of total amounts of GCF Wnt-5a to diagnose chronic periodontitis is shown in Figure 21. The area under the ROC curve for GCF Wnt-5a was 0.516 with $95 \%$ confidence interval between 0.348 and 0.684 with a non-significant $p$-value of 0.846 . In regards to TNF- $\alpha$, ROC analysis of total amounts of GCF TNF- $\alpha$ to diagnose chronic periodontitis is shown in Figure 22. The area under the ROC curve was found to be 0.642 with $95 \%$ confidence interval from 0.485 to 0.798 and a corresponding p-value of 0.086 . 
In general, protein levels of GCF SOST, Wnt-5a and TNF- $\alpha$ showed low discriminatory ability to diagnose chronic periodontitis and periodontal health.

\section{Additional analyses}

The protein total levels of SOST, Wnt-5a and TNF- $\alpha$ in sites with presence and absence

of bleeding on probing are demonstrated in Table 9. Sites with bleeding on probing exhibited statistically significantly higher median levels of SOST when compared to sites without bleeding on probing $(p=0.035)$. On the contrary, the median protein levels of Wnt$5 a(p=0.265)$ and TNF- $\alpha(p=0.194)$ were not associated with the presence or absence of bleeding on probing in the sampled sites.

Protein total levels and concentrations of SOST, Wnt-5a and TNF- $\alpha$ between males and females are shown in Table 10. Males showed statistically significantly higher median Wnt-5a levels than females $(p=0.031)$, while no differences were found between the groups in regards to total SOST levels $(p=0.642)$ and TNF- $\alpha$ levels $(p=0.777)$ as well as the concentrations of SOST $(p=0.166)$, Wnt-5a $(p=0.307)$ and TNF- $\alpha(p=0.709)$.

\section{"Strict" criteria for patient selection based on clinical periodontal parameters}

An additional analysis was performed including patients with a more "strict" definition of periodontal disease and health. In this analysis, patients with a generalized form of 
periodontitis in at least $30 \%$ of the sites with PPD $\geq 4 \mathrm{~mm}, \mathrm{CAL} \geq 3 \mathrm{~mm}$ and presence of BOP were included in a new periodontitis subgroup. In this separate analysis, patients were considered periodontally healthy when PPD ranged from 1 to $3 \mathrm{~mm}, \mathrm{CAL} \leq 1 \mathrm{~mm}$ and BOP $\leq 15 \%$. Ten patients from the initial population met the "strict" periodontitis criteria, while ten individuals from the initial healthy group were eligible for the "strict" healthy subgroup. The Shapiro-Wilk test was also utilized to assess the normality of the distribution and nonparametric comparisons were performed. The protein levels of SOST, Wnt-5a and TNF- $\alpha$ of periodontitis and healthy subgroups using "strict" criteria for diagnosing periodontal disease and health are shown in Table 11. Periodontitis subgroup exhibited higher median total amounts of SOST (140.0 pg), Wnt-5a (2.4 ng) and TNF- $\alpha$ (44.7 pg) when compared to the healthy subgroup: $78.9 \mathrm{pg}$ for SOST, $1.29 \mathrm{ng}$ for Wnt-5a and $27.3 \mathrm{pg}$ for TNF- $\alpha$. Statistical significance was reached for the comparison between the periodontitis and healthy subgroups in regards to the SOST total amounts $(p=0.002)$. Mann-Whitney $U$ tests did not reach the significant level for Wnt-5a $(p=0.075)$ and TNF$\alpha(p=0.226)$. The distributions of the total amounts of GCF SOST, Wnt-5a and TNF- $\alpha$ of the diseased and healthy sites in the strictly diagnosed periodontitis and healthy patients are presented in Figures 23, 24 and 25.

ROC analyses of the total amounts of GCF SOST, Wnt-5a and TNF- $\alpha$ were also performed to diagnose generalized chronic periodontitis using the data from the sample of the 20 strictly diagnosed periodontitis and healthy patients. The ROC curves are shown in Figure 26, 27 and 28. The area under the curve for SOST was estimated to be 0.900 with a $95 \%$ confidence interval between 0.742 and 1.000 . Protein total levels of SOST in 
GCF discriminate between generalized periodontitis and health with a $p$-value of 0.002 . When the total amounts of Wnt-5a were used to predict periodontal disease, the area under the curve was $0.740(95 \% \mathrm{Cl}: 0.517-0.963)$ with a p-value of 0.070 . With respect to TNF- $\alpha$, the area under the curve was found to be 0.660 (95\% Cl: $0.387-0.933)$ with a pvalue of 0.226 . 


\section{DISCUSSION}

The use of GCF samples aims to develop an accurate, sensitive and reproducible method of detecting the presence of periodontal disease and to predict the risk of future periodontal destruction. Although clinical measures have been utilized extensively in everyday clinical practice and the combination of clinical and radiographic examination remains the gold standard in clinical periodontology and periodontal research, these diagnostic methods are time-consuming and proper training and calibration of the examiner is needed. Clinical measures provide a history of periodontal breakdown that already has occurred. When it comes to future periodontal destruction, biomarkers can detect early disease activity or risk of disease progression which will ultimately result in earlier therapeutic intervention. However, a significant association has to be initially found in a cross-sectional manner to establish a diagnostic use prior to evaluating it for a potential prognostic value. The present study intended to assess the involvement of Wnt signaling in chronic periodontitis and to examine whether SOST and Wnt-5a in the GCF has the potential to be used as diagnostic markers of periodontal disease status. We hypothesized that sites with chronic periodontitis exhibit elevated protein levels and

concentrations of SOST, Wnt-5a and TNF-a when compared to periodontally healthy sites. In addition, a positive correlation between the examined periodontal clinical parameters including probing pocket depth and clinical attachment loss were expected to be found with the total amounts and concentrations of SOST, Wnt-5a and TNF- $\alpha$ in GCF. 
A number of sampling methods and collection times have been utilized in previous GCF studies. The method of collection and analysis significantly affects the quantity and quality of GCF samples revealing the need for standardization (Egelberg and Attstrom 1973, Persson and Page 1990, Lamster et al. 1989). Different sampling techniques, sampling times and data presentations are critical in GCF studies (Ozkavaf et al. 2000). In the present study, sampling was performed exclusively with paper strips using the same Lot number from a single manufacturer to avoid differences in the collected volume of the samples. A standardized time for collection of GCF was also applied to all sampled sites and it was set to 30 seconds. Immediate freezing of all samples was completed to prevent proteolysis and presentation of data was similarly presented for all samples. Sampled sites in the subjects with periodontitis demonstrated PPD between 5 and $12 \mathrm{~mm}$ and CAL from 4 to $13 \mathrm{~mm}$. Sites were selected based on the most severe periodontal status. The increased variability in the periodontal status of the sampled sites may be considered a limitation of the study. GCF variability in regards to volume, concentration and total amounts are reported in the literature. It is believed that this variability may be associated with the current disease activity, progression of periodontal disease, the different stages of inflammation, disease severity as well as the microbial background (Goodson 2003, Griffiths 2003, Hancock et al. 1979).

The expression of GCF data is presented both in total amounts and concentrations. However, the conclusions of the present study are primarily based on the total amount per standardized sampling time instead of concentration to eliminate the possible inherent errors in reporting concentrations. It has been shown that expressing molecules in GCF 
as total amounts is more sensitive than reporting them as a concentration (Lamster 1997). An accurate calculation of proteins in GCF is impeded by the inability to determine the precise volume of small quantities of GCF that are usually recovered from healthy periodontal tissues (Lamster et al. 1985). A standard amount of GCF has to be collected in order to express accurately the results as concentrations. In addition, there are other parameters that may affect the concentration of GCF components. Greater GCF volume has been demonstrated at mandibular than maxillary sites (Smith and Gaegan 1991) as well as at posterior when compared to anterior sites in both periodontal health and disease (Smith 1992). When GCF levels are expressed in total amount per site, the differences are less pronounced and more closely associated with the severity or status of periodontal disease than when concentrations are used (Smith 1992). The quantity of saliva collected on filter paper strips has the potential to affect the GCF volume, while this does not significantly increase the amount of protein level per sample. Therefore, in studies including GCF data, drawing conclusions based on the total activity is more appropriate than concentrations (Lamster et al. 1986).

Saliva and dental plaque are both possible contaminants of GCF (Griffiths et al. 1992). Dental plaque adhering to paper strips may lead to higher Periotron readings and therefore to a higher GCF volume (Stoller et al. 1990). In a study by Stoller and colleagues a $50 \%$ greater volume of GCF was detected when plaque was not removed from the teeth before sampling (Stoller et al. 1990). Similarly, Griffiths et al. (1992) showed that if visible plaque was left intentionally on a tooth, there was a consistent increase in GCF volume as the plaque accumulation was increased in the paper strip. Saliva may also increase the 
estimated GCF volume as well as the flow rate as a result of the $82 \%$ of water that is contained in saliva (Stoller et al. 1990, Griffiths et al. 1992). To limit the effect of plaque on GCF volume in the present investigation, plaque removal prior to GCF sampling was performed, while teeth and surrounding periodontal tissues were dried to avoid contamination of GCF with saliva.

Bone metabolism to maintain a healthy skeleton is controlled by osteoblasts and osteoclasts. SOST decreases osteoblastogenesis and osteoblastic activity antagonizing the Wnt signaling pathway ( $\mathrm{Li}$ et al. 2005, Rossini et al. 2013). SOST also promotes osteoclast differentiation and bone resorption (Wijenayaka et al. 2011), while treatment with monoclonal antibodies targeting SOST have shown enhanced bone formation, increase bone mass and bone strength ( $\mathrm{Li}$ et al. 2009, Li et al. 2010). In a cross-sectional study with peri-implantitis, peri-implant mucositis and healthy peri-implant tissues, crevicular fluid samples were collected and the concentrations of SOST were evaluated (Rakic et al. 2014). SOST showed significantly increased levels in peri-implantitis tissues when compared to peri-implant mucositis and healthy tissues suggesting a potential role as a diagnostic marker of peri-implant destruction (Rakic et al. 2014). Another crosssectional study explored the involvement of SOST in chronic periodontitis (Napimoga et al. 2014). In this study, gingival biopsies and serum were sampled from systemically healthy patients diagnosed with chronic periodontitis and periodontally healthy patients. SOST was statistically significantly upregulated in the gingival tissue and serum samples of patients with chronic periodontitis when compared to periodontally healthy patients. 
The involvement of SOST in periodontal destruction has also been supported by a study in rats in which inhibition of SOST with neutralized antibodies stimulated regeneration of the lost alveolar bone (Taut et al. 2013). Chronic periodontitis patients have also shown increased SOST levels in GCF in another prospective cohort study from Turkey (Balli et al. 2015). This study followed 54 patients with chronic periodontitis and periodontal health for 6 weeks after non-surgical periodontal therapy when GCF samples were collected and clinical examinations were performed. Non-surgical periodontal therapy resulted in decreased levels of SOST and its reduction was related to clinical improvement of a patient's periodontal status. In the present study, statistically significant correlations were identified between the total amounts of SOST and clinical parameters when expressed as full-mouth and site-specific measures $(p<0.05)$. The total amount of SOST was slightly greater in the periodontitis group (135.5 pg) when compared to the healthy group (117.9 $p g$ ), while no statistically significant difference was found $(p>0.05)$. When diseased and healthy sites in a subset of periodontitis patients were examined, non-significant results were also found. Although both results from our investigation and the study of Balli et al. used GCF samples, the findings are not in complete agreement. This may be attributed to differences in the inclusion criteria. More specifically, in the present investigation, the absence of gingival inflammation was not required for the healthy group, while Balli and co-investigators restricted their GCF sample to healthy patients with a gingival index of zero. In addition, the periodontitis group, Balli and colleagues recruited patients with more generalized disease (bone loss affecting $>30 \%$ of the existing teeth), while the range of PPD and CAL was narrower than in our investigation. Furthermore, another explanation could be the difference in the age of the patients in the periodontitis group. Our disease 
group had a mean age of 57.9 years, whereas the previous study included a much younger group which demonstrated a mean age of 39.56 years. It is possible that cases of aggressive periodontitis may have been included in the study of Balli and colleagues (2015). Differences in the study methodology also included the sampled sites and the analysis process. In particular, in the present study both single- and multi-rooted teeth were sampled and each sample was analyzed separately which is in contrast with the Balli's study that included only single-rooted teeth and the samples were pooled.

Wnt-5a suppresses bone formation by enhancing RANKL-induced osteoclastogenesis and its up-regulated secretion may also enhance the expression of inflammatory-related genes through a novel NF-kappaB-dependent regulation (Maeda et al. 2007, Zhao et al. 2015). Wnt-5a is secreted in chronic inflamed sites such as inflammatory synoviocytes. Nanbara et al. (2012) showed that Wnt-5a mRNA production is strongly induced by $P$. gingivalis LPS. In the same study, Wnt-5a mRNA was significantly up-regulated in chronic periodontitis gingival tissues as compared to healthy control tissues as a result of the periodontopathic bacteria (Nanbara et al. 2012). Similarly, a statistically significantly higher expression of Wnt-5a mRNA was found in gingival tissues from periodontally involved individuals when compared to healthy controls by a different research group (Maekawa et al. 2017). Although an increase Wnt-5a mRNA expression was found in periodontitis in comparison to health, this difference was not reflected at the protein level (Maekawa et al. 2017). The need for further studies that will evaluate Wnt-5a therapeutic potential and its suitability as a biomarker in periodontitis has been expressed in the literature (Maekawa et al. 2017, Nanbara et al. 2017). Currently, no available study has 
evaluated the protein levels of Wnt-5a in the GCF of chronic periodontitis patients. Therefore, our findings cannot be compared with the results of other research investigators.

In the present investigation, significant differences were found between the sampled sites in the periodontitis group in regards to the protein levels of Wnt-5a. Diseased sites in a group of periodontitis patients exhibited statistically significantly higher levels of Wnt-5a when compared to healthy sites of the same patients. This confirms our hypothesis that sites with periodontal destruction have increased Wnt-5a in the GCF. On the other hand, when sites in periodontitis and healthy patients were compared with respect to the total amounts of Wnt-5a, no significant differences were detected. This finding could possibly be a result of the age differences between the study groups. Evidence of differences in the expression of WNT genes between young and aged individuals has been reported in the literature when mesenchymal stem cells were obtained (Shen et al. 2009). More specifically, an increased WNT gene expression was shown in a young group when compared to an aged group. Furthermore, mature osteoblasts of aged mice have demonstrated decreased expression of Wnt-5a compared with those of young mice (Rauner et al. 2008, Becerikli et al. 2017). Therefore, the lack of association between the healthy and periodontitis group in our study may be attributed to the significantly younger age of the control as compared to the periodontitis group. Obesity has also been associated with elevated levels of Wnt-5a in humans when compared to lean controls (Schulte et al. 2012). In the present study, no information was recorded in regard to body mass index of the included population. 
TNF is considered a major inflammatory mediator with two ligands. It is produced by phagocytes and T cells in response to bacteria and especially lipopolysaccharide leading to periodontal destruction (Ebersole and Cappelli 2000). TNF- $\alpha$ affects the expression of other inflammatory mediators including IL-1, PGE2 and MMP (Ebersole and Cappelli 2000; Cekici et al. 2014). It is involved in recruiting leukocytes to inflamed tissues, vascular permeability and leakage and chemokine production (Hofmann et al. 2002, Frank et al. 1995). In the present investigation, the protein levels of TNF- $\alpha$ were similar between periodontitis and healthy patients as well as when periodontitis and healthy sites were compared in a periodontitis subgroup. In this study, a wide range of TNF- $\alpha$ levels in both periodontitis and healthy sites were also found. This may be related to the clinical variability among the sampled sites. The lack of association between periodontal status and TNF- $\alpha$ GCF levels could be explained by the disease activity at the time of the sampling. Perhaps the active inflammatory process due to the chronicity of the disease might have weakened.

Previous studies have shown contradictory results. In a study by Reis et al. (2014) TNF- $\alpha$ levels were significantly higher in diseased sites compared to non-diseased sites (Reis et al. 2014). In comparison to our study, Reis et al. considered only PPD to define chronic periodontal disease while it is unclear the criteria used for non-diseased sites. Gokul and colleagues (2012) compared gingivitis, chronic periodontitis and healthy individuals showing that there was a highly significant difference between healthy subjects when compared to the other two groups, while no significant differences were detected between 
gingivitis and periodontitis groups (Gokul et al. 2014). TNF- $\alpha$ may also be present in normal gingival tissues and may be related to physiological activities. Healthy gingival tissues exhibit a low number of macrophages and mononuclear cells and GCF includes neutrophils in a healthy periodontium which may explain the presence of TNF- $\alpha$ in healthy sites (Page 1991). The variability of findings among the studies may be attributed to the different inclusion criteria used for the groups. In the present investigation, no restriction was applied to the healthy patients as far as gingival inflammation was concerned. Therefore, patients included in the healthy group might also have gingival inflammation. As a result, presence of high numbers of inflammatory cells in the connective tissue and gingival crevice may up-regulate TNF- $\alpha$ upon stimulation by the bacterial products (Page 1991). High GCF TNF-a levels have also been found in a healthy periodontium (Ulker et al. 2008). Rossomando and colleagues (1990) suggested that TNF- $\alpha$ may be found in sites prior to clinically observable disease which may explain these results.

In general, differences in the findings among studies utilizing GCF samples may be attributed to different parameters. First of all, the vast majority of the studies have used paper strips which are efficient and easy to insert into the gingival sulcus or periodontal pocket. This method can lead to a higher absorption of the GCF than using paper points. In a study by Guentch and colleagues, the use of paper strips resulted in higher cytokine levels when compared to paper points (Guentch et al. 2011). In addition, the time of GCF collection varies from 30 seconds to 1 minute. In the present study, paper strips were inserted into the sulcus or pocket for 30 seconds in order to reduce the risk of blood or saliva contamination. Moreover, different GCF analytic methods have been employed. 
The majority of the GCF studies have used the ELISA technique, while others have performed the immunofluorometric assay (IFMA) which was shown by Leppilahti et al. (2014) to be more accurate than using ELISA. Another important parameter is the use of different diagnostic criteria for periodontal disease and health as well as the collection of a limited number of samples from each individual. The use of different inclusion criteria based on disease status increases the heterogeneity of the studies and does not allow for accurate comparisons between them which may explain the controversial findings. Collection of a small number of samples per patient may not permit a precise evaluation and analysis of the protein total levels or concentrations associated with periodontal disease and health due to the fact that the development of periodontitis is site-specific (Fujita et al. 2012).

In an attempt to compare the protein levels of SOST, Wnt-5a and TNF- $\alpha$ in GCF of diseased and healthy sites from patients with a generalized form of chronic periodontitis and periodontal health, "strict" criteria were used to define periodontitis and health. PPD, $\mathrm{CAL}$ and BOP were taken into consideration and patients with $\geq 30 \%$ of the sites with PPD $\geq 4 \mathrm{~mm}, \mathrm{CAL} \geq 3 \mathrm{~mm}$ and presence of BOP were included in a periodontitis subgroup $(\mathrm{n}=10)$. Similarly, patients with PPD $\leq 3 \mathrm{~mm}, \mathrm{CAL} \leq 1 \mathrm{~mm}$ and $\mathrm{BOP} \leq 15 \%$ were included in a healthy subgroup $(n=10)$. The statistical analysis revealed that the protein levels of SOST, Wnt-5a and TNF- $\alpha$ were higher in the periodontitis group when compared to the healthy. The results obtained from this subgroup analysis confirmed the initial hypothesis of the present investigation. However, only the comparison between the two groups in regards to SOST total amounts reached the significant level $(p=0.002)$. Future studies 
should include a greater number of patients with a generalized form of chronic periodontitis ( $\geq 30 \%$ of the sites), and BOP should also be considered when periodontitis and healthy individuals are recruited.

Self-reported medical history and smoking status was utilized in the present investigation. Smoking status was recorded as an independent variable, while medical history was used to determine the eligibility of the patients to participate in the study based on the inclusion and exclusion criteria. Laboratory and/or physical examinations were not possible to be conducted in the Graduate Periodontology clinic of the University of Minnesota School of Dentistry, but self-reported measures have been used extensively in the literature and presents a cost effective and reliable method (Wada et al. 2009, Kehoe et al. 1994). Using patient self-reported data may be relatively faster, easier and less expensive than requesting a medical record examination, but there are limitations due to bias in patient recall, untruthful responses as a result of social desirability and a patient's lack of knowledge (Newell et al. 1999). However, self-reported measures are accurate for chronic conditions and routine screening exams and may also provide valuable information for broad measures of population prevalence (Martin et al. 2000). Self-reported history of hypertension, diabetes and angina have shown generally high validity, whereas selfreported hypercholesterolemia should be used with caution (Natarajan et al. 2002, Lampe et al. 1999, Robinson et al. 1997). It is generally believed that the variability by medical conditions may be derived from poor communication between physicians and patients due to the fact that diseases with clear diagnostic criteria such as diabetes, hypertension and myocardial infarction demonstrated higher rates of agreement than those that are difficult 
for the patient to understand such as heart failure (Okura et al. 2004). It should also be expected that patients with impaired memory, psychological problems, psychiatric disorders and distortion of reality may result in decreased accuracy of a self-reported history (Johnson-Greene et al. 1997).

Radiographic evaluation is commonly used in confirming a periodontal diagnosis and provide additional information, essential in treatment planning. Radiographs are of paramount importance in determining the extent and severity of alveolar bone loss and detecting osseous lesions (Pihlstrom 2001). Widely used radiographic modalities have been utilized and include bitewing, periapical and panoramic radiography. A full-mouth intraoral series of radiographs is the appropriate radiographic method for evaluation of periodontal disease and especially generalized disease or a history of extensive dental treatment. Panoramic radiographs exhibit limitations such as superimposition and distortion of structures, blurring of anatomical structures, while the determination of bone levels and defect depths is limited. A panoramic radiograph may be used in conjunction with bitewing radiographs and selected periapical radiographs as an alternative option for an intraoral full-mouth series of intraoral radiographs (Mol 2004). However, in the present investigation, a full-mouth series of periapical radiographs in combination with horizontal or vertical bitewings was utilized to determine the eligibility of the examined individual for the study. Our decision is in agreement with the American Dental Association's recommendations reporting that new patients that are adolescents with permanent dentition or adults (dentate or partially edentulous) should be exposed to a full-mouth intraoral radiographic examination when there is clinical evidence of generalized oral 
disease or a history of extensive dental treatment (American Dental Association 2012). Bitewing radiography was also required in the present study because its sensor is placed parallel to the tooth leading to the most accurate radiographic projection of the level of the alveolar crest (Eley and Cox 1998, White et al. 2001).

The normal bone height in relation to the CEJ varies between $1 \mathrm{~mm}$ and $3 \mathrm{~mm}$, while a distance of $2 \mathrm{~mm}$ is more widely accepted in studies of patients with a healthy periodontium (Wong et al. 2007, Gargiulo et al. 1961, Lennon and Davies 1974). In the present study, the distance from the alveolar bone to the CEJ was subjectively measured and the overall root length from the CEJ to the root tip was also measured. The percentage of bone loss was then expressed as a ratio of the amount of bone loss to the overall root length taking into consideration the normal bone level and then equated to the severity of bone loss. Individuals were included in the periodontitis group if $40 \%$ of bone loss was radiographically evident, while no radiographically detected bone loss was needed for the healthy group. Direct digital radiography compared to conventional film-based radiography has shown numerous advantages such as adjustable contrast and brightness, less working time and less radiation (Wenzel 1993). In the present study, direct digital radiographs were utilized to eliminate the limitations found with conventional modalities. 


\section{CONCLUSIONS}

In conclusion, the aim of developing new diagnostics in Periodontology remains important to identify patients at risk of periodontal disease onset and progression. Based on this research project, the following conclusions can be made:

1. SOST, Wnt-5a and TNF- $\alpha$ are detectable in the GCF of periodontally healthy and compromised sites.

2. Sites with chronic periodontitis showed similar protein total amounts of SOST, Wnt$5 a$ and TNF- $\alpha$ when compared to sites with healthy periodontium.

3. The concentrations of SOST, Wnt-5a and TNF- $\alpha$ demonstrated increased variability among the sampled sites and significant differences should be considered with caution.

4. Diseased sites in patients with chronic periodontitis exhibited significantly elevated total protein levels of Wnt-5a when compared to healthy sites in the same patients. No significant differences were detected with SOST and TNF- $\alpha$.

5. SOST, Wnt-5a and TNF- $\alpha$ demonstrated low diagnostic value of chronic periodontitis.

6. In a subgroup analysis of patients with a generalized form of chronic periodontitis and periodontal health, the total protein levels of SOST were significantly higher in periodontitis than in healthy sites. No significant differences were found in regards to Wnt-5a and TNF- $\alpha$. However, a small sample group $(n=10)$ was used for this subgroup analysis. 
7. Increased diagnostic value was detected when patients with a generalized moderate and severe form of chronic periodontitis were evaluated.

8. GCF SOST total amounts were significantly positively correlated or demonstrated a trend towards a positive correlation with the full mouth mean of PPD, CAL and the percentage of sites with PPD 4-6 mm. A trend towards a negative correlation was found between SOST and sites with a CAL 0-2 mm.

9. Future studies should employ "strict" diagnostic criteria and recruit patients diagnosed with a generalized moderate and severe form of chronic periodontitis and periodontal health. These studies should aim to further evaluate the findings of the subgroup analysis in the present study and determine the diagnostic value of screening patients with moderate and severe chronic periodontitis.

10. Longitudinal studies should evaluate the prognostic value of SOST, Wnt-5a and TNF- $\alpha$ in chronic periodontitis. 
Table 1: Demographic characteristics of the total population as well as the periodontitis and healthy groups

\begin{tabular}{|c|c|c|c|c|}
\hline Characteristics & $\begin{array}{c}\text { Periodontitis } \\
\text { group } \\
(\mathrm{N}=25-50 \%)\end{array}$ & $\begin{array}{c}\text { Healthy } \\
\text { group } \\
(\mathrm{N}=25-50 \%)\end{array}$ & $\begin{array}{c}\text { Total } \\
\text { population } \\
(\mathrm{N}=50-100 \%)\end{array}$ & $p$-value \\
\hline $\begin{array}{l}\text { Age } \\
\text { Mean (SD) }\end{array}$ & $57.9(12.6)$ & $28.9(10.2)$ & $43.4(18.5)$ & $<0.001^{\dagger}$ \\
\hline $\begin{array}{l}\text { Gender } \\
\text { Males (\%) } \\
\text { Females (\%) }\end{array}$ & $\begin{array}{l}12(24) \\
13(26)\end{array}$ & $\begin{array}{l}6(12) \\
19(38)\end{array}$ & $\begin{array}{l}18(36) \\
32(64)\end{array}$ & $0.077^{\ddagger}$ \\
\hline $\begin{array}{l}\text { Race } \\
\text { Caucasians (\%) } \\
\text { African-Americans (\%) } \\
\text { Asians (\%) } \\
\text { Hispanics (\%) }\end{array}$ & $\begin{array}{l}18(36) \\
5(10) \\
0(0) \\
2(4)\end{array}$ & $\begin{array}{l}22(44) \\
1(2) \\
2(4) \\
0(0)\end{array}$ & $\begin{array}{l}40(80) \\
6(12) \\
2(4) \\
2(4)\end{array}$ & $0.070^{\ddagger}$ \\
\hline $\begin{array}{l}\text { Smoking status } \\
\text { Never smokers (\%) } \\
\text { Ex-smokers (\%) } \\
\text { Current smokers (\%) }\end{array}$ & $\begin{array}{l}10(20) \\
6(12) \\
9(18)\end{array}$ & $\begin{array}{l}25(50) \\
0(0) \\
0(0)\end{array}$ & $\begin{array}{l}35(70) \\
6(12) \\
9(18)\end{array}$ & $<0.001^{\ddagger}$ \\
\hline $\begin{array}{l}\text { Type of smoking } \\
\text { Cigarettes (\%) } \\
\text { Cigars (\%) } \\
\text { Pipe (\%) } \\
\text { Chewing tobacco (\%) }\end{array}$ & $\begin{array}{l}9(100) \\
0(0) \\
0(0) \\
0(0)\end{array}$ & $\begin{array}{l}0(0) \\
0(0) \\
0(0) \\
0(0)\end{array}$ & $\begin{array}{l}9(100) \\
0(0) \\
0(0) \\
0(0)\end{array}$ & $1.00^{\ddagger}$ \\
\hline $\begin{array}{l}\text { Amount of smoking } \\
<10 \text { cig/day }(\%) \\
10-20 \text { cig/day }(\%) \\
>20 \text { cig/day }(\%)\end{array}$ & $\begin{array}{l}5(55.5) \\
4(44.4) \\
1(11.1)\end{array}$ & $\begin{array}{l}0(0) \\
0(0) \\
0(0)\end{array}$ & $\begin{array}{l}5(55.5) \\
4(44.4) \\
1(11.1)\end{array}$ & $1.00^{\ddagger}$ \\
\hline
\end{tabular}

* Statistical significance between study groups with $p$-value $<0.05$.

† T-test was used to compare the mean age between the study groups.

$¥$ Chi-square test was used to compare categorical variables including gender, race, smoking status, type of smoking, amount of smoking between the study groups. 
Table 2: Periodontal status of the total population as well as the periodontitis and healthy group.

\begin{tabular}{|l|l|l|l|l|}
\hline \multicolumn{1}{|c|}{ Characteristics } & $\begin{array}{c}\text { Periodontitis } \\
\text { group } \\
(\mathrm{N}=25-50 \%)\end{array}$ & $\begin{array}{c}\text { Healthy } \\
\text { group } \\
(\mathrm{N}=25-50 \%)\end{array}$ & $\begin{array}{c}\text { Total } \\
\text { population } \\
(\mathrm{N}=50-100 \%)\end{array}$ & $\mathrm{p}^{\text {-value }}$ \\
\hline PPD (mm) & $3.57 \pm 1.20$ & $1.90 \pm 0.34$ & $2.73 \pm 1.21$ & $<0.001$ \\
\hline CAL (mm) & $3.44 \pm 1.83$ & $0.09 \pm 0.09$ & $1.76 \pm 2.12$ & $<0.001$ \\
\hline BOP (\%) & $42.09 \pm 30.34$ & $12.71 \pm 15.72$ & $27.40 \pm 28.14$ & $<0.001$ \\
\hline PI (\%) & $25.87 \pm 32.21$ & $2.74 \pm 4.57$ & $14.30 \pm 25.59$ & 0.001 \\
\hline Missing teeth & $4.04 \pm 3.76$ & $0.76 \pm 1.36$ & $2.40 \pm 3.25$ & $<0.001$ \\
\hline PPD=1-3 mm (\%) & $61.43 \pm 22.73$ & $97.43 \pm 4.66$ & $79.42 \pm 24.38$ & $<0.001$ \\
\hline PPD=4-6 mm (\%) & $28.92 \pm 15.65$ & $2.57 \pm 4.66$ & $15.74 \pm 17.54$ & $<0.001$ \\
\hline PPD $\geq 7 \mathrm{~mm}(\%)$ & $9.35 \pm 14.06$ & $0.00 \pm 0.00$ & $4.68 \pm 10.92$ & 0.002 \\
\hline CAL=0-2 mm (\%) & $43.21 \pm 27.58$ & $100.00 \pm 0.00$ & $71.61 \pm 34.57$ & $<0.001$ \\
\hline CAL=3-4 mm (\%) & $28.35 \pm 13.02$ & $0.00 \pm 0.00$ & $14.17 \pm 16.98$ & $<0.001$ \\
\hline CAL $\geq 5 \mathrm{~mm}(\%)$ & $28.44 \pm 25.12$ & $0.00 \pm 0.00$ & $14.22 \pm 22.70$ & $<0.001$ \\
\hline
\end{tabular}

* Statistical significance between study groups with $p$-value $<0.05$. T-test was used to compare the mean values between the study groups.

Abbreviations:

PPD: Probing Pocket Depth

CAL: Clinical Attachment Level

BOP: Bleeding On Probing

PI: Plaque index 
Table 3: Probing pocket depth and clinical attachment level of sampled sites in healthy and periodontitis group.

\begin{tabular}{|l|l|l|l|l|l|l|}
\hline $\begin{array}{l}\text { Clinical } \\
\text { parameters }\end{array}$ & $\begin{array}{l}\text { Site-periodontal } \\
\text { status } \\
\text { (Diseased/healthy) }\end{array}$ & $\mathrm{N}$ & $\begin{array}{l}\text { Mean } \\
(\mathrm{mm})\end{array}$ & $\begin{array}{l}\text { Standard } \\
\text { deviation } \\
(\mathrm{mm})\end{array}$ & $\begin{array}{l}\text { Minimum } \\
(\mathrm{mm})\end{array}$ & $\begin{array}{l}\text { Maximum } \\
(\mathrm{mm})\end{array}$ \\
\hline PPD & $\begin{array}{l}\text { Diseased in } \\
\text { periodontitis }\end{array}$ & 25 & 6.56 & 1.73 & 5.00 & 12.00 \\
\cline { 2 - 7 } & $\begin{array}{l}\text { Healthy in } \\
\text { periodontitis }\end{array}$ & 14 & 2.50 & 0.52 & 2.00 & 3.00 \\
\cline { 2 - 7 } & $\begin{array}{l}\text { Healthy in health } \\
\text { CAL }\end{array}$ & 25 & 2.56 & 0.77 & 1.00 & 4.00 \\
& $\begin{array}{l}\text { Diseased in } \\
\text { periodontitis }\end{array}$ & 25 & 7.24 & 2.52 & 4.00 & 13.00 \\
\cline { 2 - 7 } & $\begin{array}{l}\text { Healthy in } \\
\text { periodontitis }\end{array}$ & 14 & 0.86 & 0.77 & 0.00 & 2.00 \\
\cline { 2 - 7 } & Healthy in health & 25 & 0.12 & 0.33 & 0.00 & 1.00 \\
\hline
\end{tabular}

Abbreviations:

PPD: Probing Pocket Depth

CAL: Clinical Attachment Level 
Table 4: Bleeding on probing of sampled sites in healthy and periodontitis group.

\begin{tabular}{|l|l|l|l|}
\hline $\begin{array}{l}\text { Site-Periodontal } \\
\text { status } \\
\text { (Diseased/healthy) }\end{array}$ & \multicolumn{2}{|c|}{ Bleeding on probing } & \multirow{2}{*}{ Total } \\
\cline { 2 - 4 } & Yes (\%) & No (\%) & $25(100.0)$ \\
\hline $\begin{array}{l}\text { Diseased in } \\
\text { periodontitis }\end{array}$ & $23(92.0)$ & $2(8.0)$ & $14(100.0)$ \\
\hline $\begin{array}{l}\text { Healthy in } \\
\text { periodontitis }\end{array}$ & $3(21.4)$ & $11(78.6)$ & $25(100.00)$ \\
\hline Healthy in health & $5(20.0)$ & $20(80.0)$ & $64(100.0)$ \\
\hline Total & $31(48.4)$ & $33(51.6)$ & \\
\hline
\end{tabular}


Table 5: Protein levels and concentrations of SOST, Wnt-5a and TNF- $\alpha$ of periodontitis and healthy patients.

\begin{tabular}{|c|c|c|c|c|c|c|c|}
\hline 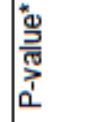 & : & & 莒 & 登 & 离 & סू & 咅 \\
\hline 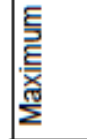 & $\overline{\frac{N}{N}}$ & 亩 & & 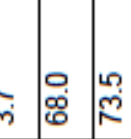 & 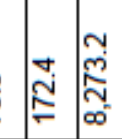 & 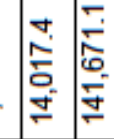 & ר్ \\
\hline 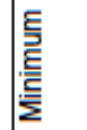 & ஜ & : & $\overbrace{0}^{\infty}$ & 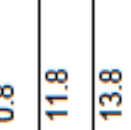 & תู & 占 & $\underset{0}{8}$ \\
\hline 豪 & فُ & 界 & $\left.\right|^{4}$ & J & : & 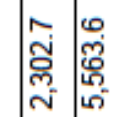 & (ָ) \\
\hline 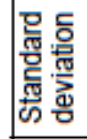 & ه్n & : & 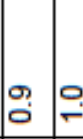 & 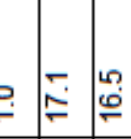 & 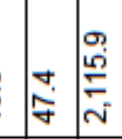 & 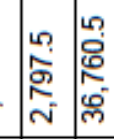 & ja \\
\hline $\begin{array}{l}\text { 急 } \\
\text { 这 }\end{array}$ & ف․ & $\hat{\tilde{n}}$ & $\bar{n}$ & 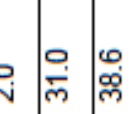 & & 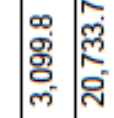 & $\frac{-}{\bar{F}}$ \\
\hline$z$ & $\stackrel{2}{2}$ & 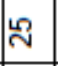 & $\stackrel{2}{2}$ & ง & ڤ & 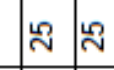 & 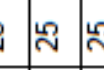 \\
\hline 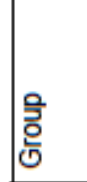 & 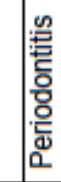 & 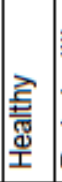 & 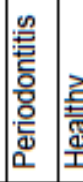 & 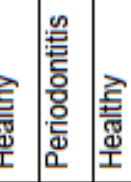 & 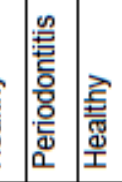 & 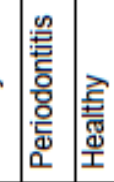 & 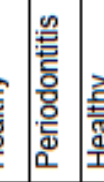 \\
\hline 穿 & 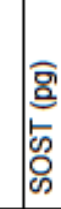 & & 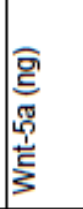 & 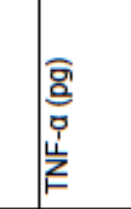 & 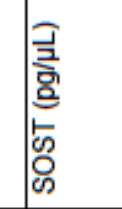 & 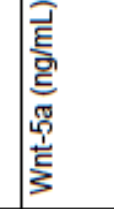 & 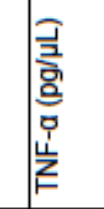 \\
\hline 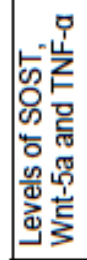 & 遆 & & & & 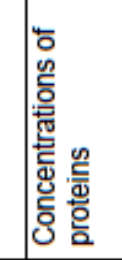 & & \\
\hline
\end{tabular}

* Statistical significance between study groups with $p$-value $<0.05$. MannWhitney $U$ test was used to compare protein total amounts and concentrations between healthy patient sites and diseased sites in periodontitis patients. 
Table 6: Protein levels and concentrations of SOST, Wnt-5a and TNF- $\alpha$ of diseased sites and healthy sites in periodontitis patients

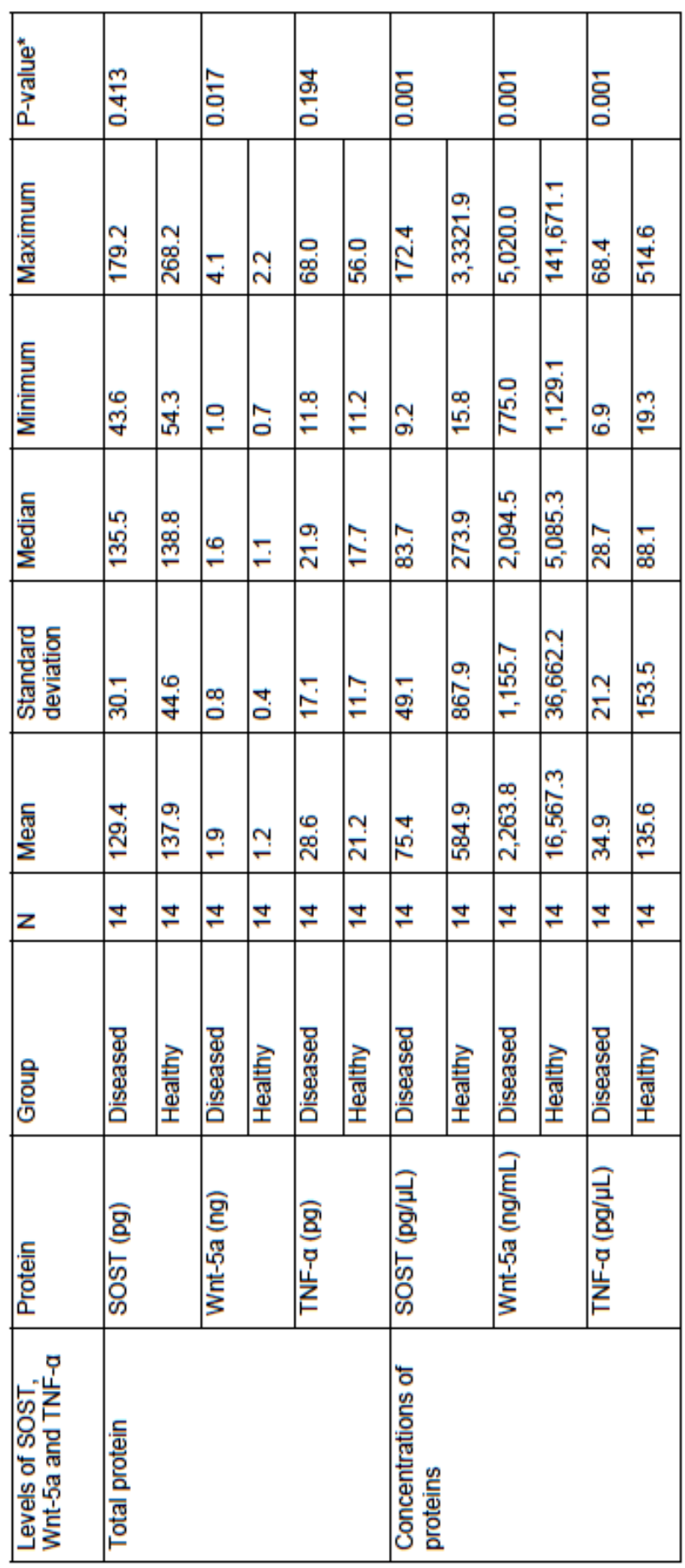

* Statistical significance between study groups with $p$-value $<0.05$. Wilcoxon signed-rank test was utilized to compare diseased sites and healthy sites in a subset of periodontitis patients that exhibited both diseased and healthy sites. 
Table 7: Spearman's rank correlations (rho) between protein total levels of SOST, Wnt-5a and TNF- $\alpha$ with the periodontal clinical and age parameters.

\begin{tabular}{|l|l|l|l|}
\hline \multirow{2}{*}{ Parameters } & \multicolumn{3}{|c|}{ Protein levels } \\
\cline { 2 - 4 } & SOST & Wnt-5a & TNF- $\alpha$ \\
\hline PPD (full mouth) & $\begin{array}{l}r=0.29 \\
p=0.044\end{array}$ & $\begin{array}{l}r=0.01 \\
p=0.936\end{array}$ & $\begin{array}{l}r=-0.26 \\
p=0.071\end{array}$ \\
\hline CAL (full mouth) & $r=0.36$ & $r=0.10$ & $r=-0.21$ \\
& $p=0.010$ & $p=0.507$ & $p=0.134$ \\
\hline PPD (sampled sites) & $r=0.30$ & $r=-0.08$ & $r=-0.29$ \\
& $p=0.037$ & $p=0.589$ & $p=0.039$ \\
\hline CAL (sampled sites) & $r=0.21$ & $r=0.01$ & $r=-0.26$ \\
& $p=0.139$ & $p=0.950$ & $p=0.073$ \\
\hline PPD=1-3 mm & $r=-0.34$ & $r=-0.04$ & $r=0.26$ \\
& $p=0.017$ & $p=0.773$ & $p=0.066$ \\
\hline PPD=4-6 mm & $r=0.33$ & $r=0.04$ & $r=-0.23$ \\
& $p=0.018$ & $p=0.771$ & $p=0.107$ \\
\hline PPD $\geq 7 \mathrm{~mm}$ & $r=0.24$ & $r=0.03$ & $r=-0.27$ \\
& $p=0.100$ & $p=0.844$ & $p=0.059$ \\
\hline CAL=0-2 mm & $r=-0.28$ & $r=-0.14$ & $r=0.16$ \\
& $p=0.052$ & $p=0.325$ & $p=0.265$ \\
\hline CAL=3-4 mm & $r=0.26$ & $r=0.06$ & $r=-0.16$ \\
& $p=0.067$ & $p=0.666$ & $p=0.254$ \\
\hline CAL $\geq 5 \mathrm{~mm}$ & $r=0.26$ & $r=0.12$ & $r=-0.18$ \\
& $p=0.070$ & $p=0.402$ & $p=0.219$ \\
\hline Age & $r=0.27$ & $r=-0.01$ & $r=-0.28$ \\
& $p=0.055$ & $p=0.962$ & $p=0.052$ \\
\hline
\end{tabular}

Abbreviations:

PPD: Probing Pocket Depth

CAL: Clinical Attachment Level 
Table 8: Spearman's rank correlation (rho) between concentrations of SOST, Wnt-5a and TNF- $\alpha$ with the periodontal clinical and age parameters.

\begin{tabular}{|l|l|l|l|}
\hline \multirow{2}{*}{ Parameters } & \multicolumn{3}{|c|}{ Concentrations } \\
\cline { 2 - 4 } & SOST & Wnt-5a & TNF- $\alpha$ \\
\hline PPD (full-mouth) & $\begin{array}{l}r=-0.49 \\
p=0.001\end{array}$ & $\begin{array}{l}r=-0.39 \\
p=0.006\end{array}$ & $\begin{array}{l}r=-0.51 \\
p=0.001\end{array}$ \\
\hline CAL (full-mouth) & $\begin{array}{l}r=-0.51 \\
p=0.001\end{array}$ & $\begin{array}{l}r=-0.44 \\
p=0.001\end{array}$ & $\begin{array}{l}r=-0.58 \\
p<0.001\end{array}$ \\
\hline PPD (sampled sites) & $\begin{array}{l}r=-0.52 \\
p<0.001\end{array}$ & $\begin{array}{l}r=-0.39 \\
p=0.006\end{array}$ & $\begin{array}{l}r=-0.49 \\
p=0.001\end{array}$ \\
\hline CAL (sampled sites) & $r=-0.57$ & $r=-0.38$ & $r=-0.52$ \\
& $p<0.001$ & $p=0.007$ & $p<0.001$ \\
\hline PPD=1-3 mm & $r=0.47$ & $r=0.37$ & $r=0.53$ \\
& $p=0.001$ & $p=0.008$ & $p<0.001$ \\
\hline PPD=4-6 mm & $r=-0.46$ & $r=-0.33$ & $r=-0.47$ \\
& $p=0.001$ & $p=0.021$ & $p=0.001$ \\
\hline PPD $\geq 7 \mathrm{~mm}$ & $r=-0.48$ & $r=-0.38$ & $r=-0.52$ \\
& $p<0.001$ & $p=0.007$ & $p=0.001$ \\
\hline CAL=0-2 mm & $r=0.61$ & $r=0.34$ & $r=0.55$ \\
& $p<0.001$ & $p=0.015$ & $p<0.001$ \\
\hline CAL=3-4 mm & $r=-0.59$ & $r=-0.34$ & $r=-0.54$ \\
& $p<0.001$ & $p=0.016$ & $p<0.001$ \\
\hline CAL $\geq 5 \mathrm{~mm}$ & $r=-0.63$ & $r=-0.38$ & $r=-0.55$ \\
& $p<0.001$ & $p=0.006$ & $p<0.001$ \\
\hline Age & $r=-0.47$ & $r=-0.44$ & $r=-0.52$ \\
& $p=0.001$ & $p=0.001$ & $p<0.001$ \\
\hline
\end{tabular}

Abbreviations:

PPD: Probing Pocket Depth

CAL: Clinical Attachment Level 
Table 9: Protein total levels of SOST, Wnt-5a and TNF- $\alpha$ in sites with presence and absence of bleeding on probing

\begin{tabular}{|l|l|l|l|}
\hline \multirow{2}{*}{ Protein total levels } & \multicolumn{2}{|c|}{ Bleeding on probing } & \multirow{2}{*}{-value $^{*}$} \\
\cline { 2 - 3 } & $\begin{array}{l}\text { Presence } \\
(\mathrm{n}=28)\end{array}$ & $\begin{array}{l}\text { Absence } \\
(\mathrm{n}=22)\end{array}$ & \\
\hline $\begin{array}{l}\text { SOST }(\mathrm{pg}) \\
\text { Mean } \pm \text { SD } \\
\text { (Median) }\end{array}$ & $\begin{array}{l}135.7 \pm 36.3 \\
(135.5)\end{array}$ & $\begin{array}{l}122.1 \pm 52.2 \\
(115.0)\end{array}$ & 0.035 \\
\hline $\begin{array}{l}\text { Wnt-5a }(\mathrm{ng}) \\
\begin{array}{l}\text { Mean } \pm \text { SD } \\
\text { (Median) }\end{array}\end{array}$ & $\begin{array}{l}1.9 \pm 0.9 \\
(1.9)\end{array}$ & $\begin{array}{l}2.2 \pm 1.0 \\
(2.5)\end{array}$ & 0.265 \\
\hline $\begin{array}{l}\text { TNF- } \alpha(\mathrm{pg}) \\
\text { Mean } \pm \text { SD } \\
\text { (Median) }\end{array}$ & $\begin{array}{l}32.2 \pm 17.0 \\
(27.3)\end{array}$ & $\begin{array}{l}38.2 \pm 17.1 \\
(38.0)\end{array}$ & 0.194 \\
\hline
\end{tabular}

* Statistical significance between sites with and without bleeding on probing with $p$-value $<0.05$. Mann-Whitney $U$ test was used to compare the median values between the groups. 
Table 10: Protein levels and concentrations of SOST, Wnt-5a and TNF- $\alpha$ with respect to gender

\begin{tabular}{|c|c|c|c|c|}
\hline \multirow{2}{*}{$\begin{array}{l}\text { Levels of SOST, } \\
\text { Wnt-5a and TNF- } \alpha\end{array}$} & \multirow[t]{2}{*}{ Proteins } & \multicolumn{2}{|c|}{ Gender } & \multirow[t]{2}{*}{ p-value* } \\
\hline & & $\begin{array}{l}\text { Males } \\
(n=18)\end{array}$ & $\begin{array}{l}\text { Females } \\
(n=32)\end{array}$ & \\
\hline \multirow[t]{3}{*}{ Total protein } & $\begin{array}{l}\text { SOST }(p g) \\
\text { Mean } \pm \text { SD } \\
\text { (Median) }\end{array}$ & $\begin{array}{c}133.6 \pm 48.2 \\
(130.7)\end{array}$ & $\begin{array}{c}127.6 \pm 42.2 \\
(135.0)\end{array}$ & 0.642 \\
\hline & $\begin{array}{l}\text { Wnt-5a (ng) } \\
\text { Mean } \pm \text { SD } \\
\text { (Median) }\end{array}$ & $\begin{array}{c}2.5 \pm 1.1 \\
(2.5)\end{array}$ & $\begin{array}{l}1.8 \pm 0.8 \\
(1.6)\end{array}$ & 0.031 \\
\hline & $\begin{array}{l}\text { TNF- } \alpha(\mathrm{pg}) \\
\text { Mean } \pm \text { SD } \\
\text { (Median) }\end{array}$ & $\begin{array}{c}34.0 \pm 17.3 \\
(28.7)\end{array}$ & $\begin{array}{c}35.3 \pm 17.3 \\
(32.9)\end{array}$ & 0.777 \\
\hline \multirow[t]{3}{*}{$\begin{array}{l}\text { Concentrations of } \\
\text { proteins }\end{array}$} & $\begin{array}{l}\text { SOST }(\mathrm{pg} / \mu \mathrm{L}) \\
\text { Mean } \pm \mathrm{SD} \\
\text { (Median) }\end{array}$ & $\begin{array}{c}575.6 \pm 1750.1 \\
(92.6)\end{array}$ & $\begin{array}{c}506.0 \pm 1454.2 \\
(147.9)\end{array}$ & 0.166 \\
\hline & $\begin{array}{l}\text { Wnt-5a } \\
\text { (ng/mL) } \\
\text { Mean } \pm \text { SD } \\
\text { (Median) }\end{array}$ & $\begin{array}{c}13,950.4 \pm 31075.5 \\
(2559.8)\end{array}$ & $\begin{array}{c}10,772.8 \pm 25383.4 \\
(3502.3)\end{array}$ & 0.307 \\
\hline & $\begin{array}{l}\text { TNF- } \alpha \\
(p g / \mu L) \\
\text { Mean } \pm \text { SD } \\
\text { (Median) }\end{array}$ & $\begin{array}{c}320.5 \pm 834.6 \\
(62.5)\end{array}$ & $\begin{array}{c}276.1 \pm 599.9 \\
(54.9)\end{array}$ & 0.709 \\
\hline
\end{tabular}

* Statistical significance between males and females with $p$-value $<0.05$. Mann-Whitney $\mathrm{U}$ test was used to compare the median values between the groups. 
Table 11: Protein total levels of SOST, Wnt-5a and TNF- $\alpha$ of periodontitis and healthy groups using "strict" criteria for diagnosing periodontal disease and health.

\begin{tabular}{|c|c|c|c|c|c|c|}
\hline בֶ, & ర్̊口 & & 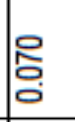 & & స్ & \\
\hline 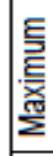 & $\overline{\grave{N}}$ & 䔡 & $\underset{\forall}{F}$ & m & జ్రి & o \\
\hline 点 & $\stackrel{\infty}{\stackrel{m}{\rightleftarrows}}$ & జூণ & $\stackrel{m}{-}$ & $\infty$ & $\stackrel{m}{\stackrel{m}{*}}$ & $\stackrel{2}{\circ}$ \\
\hline $\begin{array}{l}\text { 愛 } \\
\text { एक }\end{array}$ & 兑 & @ & i & $\stackrel{m}{?}$ & 过 & $\underset{\sim}{\sim}$ \\
\hline 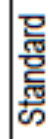 & চ্লি & & $\digamma$ & ? & $\stackrel{0}{\circ}$ & 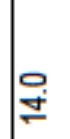 \\
\hline 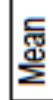 & 菖 & 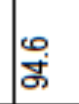 & N & $\stackrel{9}{\circ}$ & 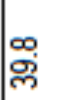 & 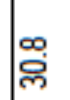 \\
\hline$z$ & 으 & 우 & 우 & 으 & 으 & 으 \\
\hline 을 & 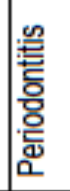 & 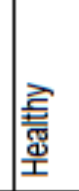 & 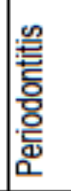 & 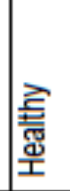 & 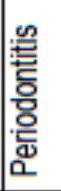 & 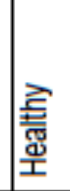 \\
\hline 홍 & 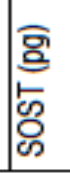 & & 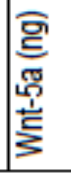 & & 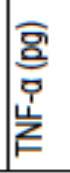 & \\
\hline
\end{tabular}

* Statistical significance between study groups with $\mathrm{p}$-value $<0.05$. Mann-Whitney $U$ test was used to compare protein total amounts between periodontally healthy $(n=10)$ patient sites and diseased sites in periodontitis patients $(n=10)$ using "strict" diagnostic criteria. 
Figure 1: Bone remodeling cycle

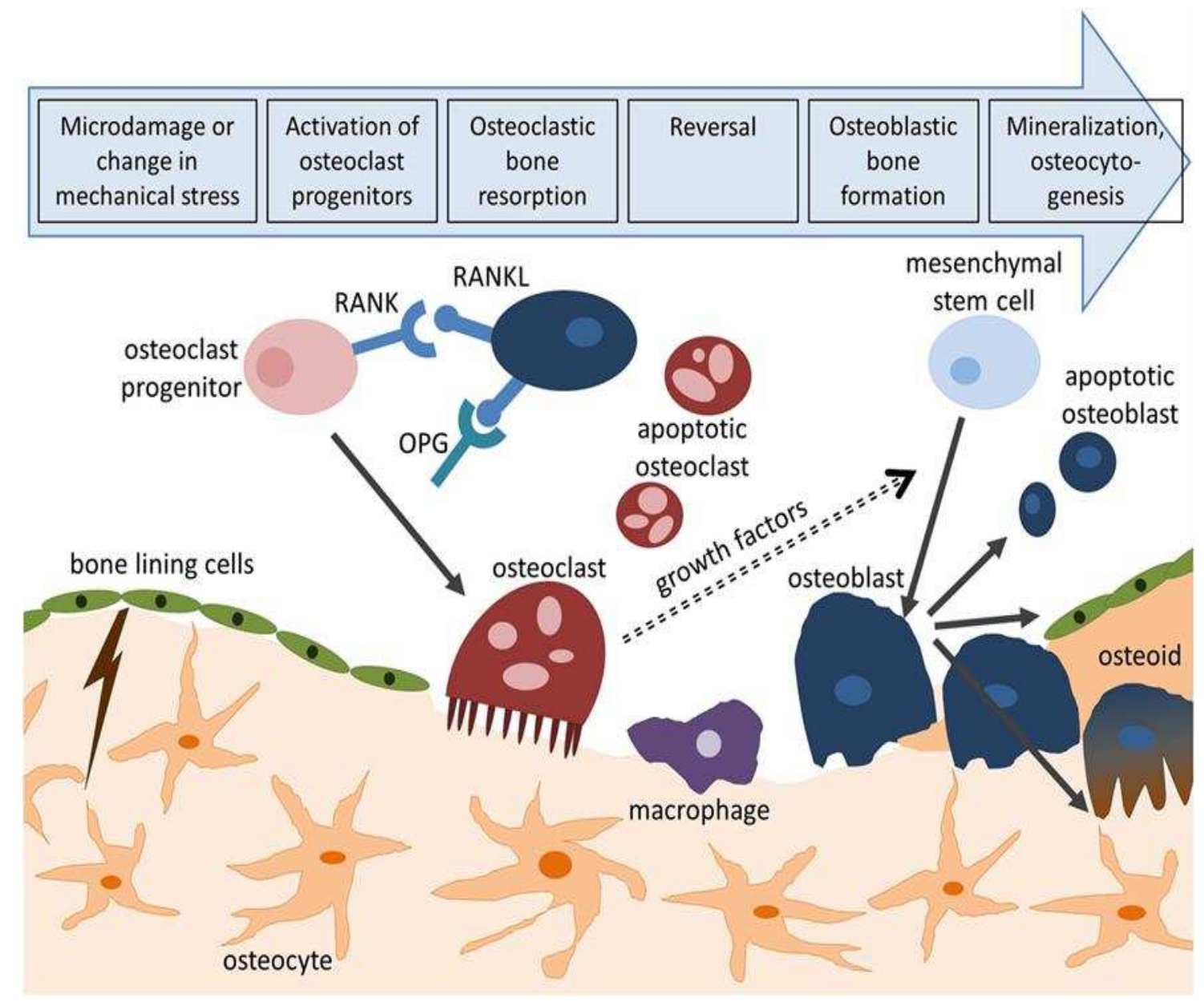

Adapted from: Wittkowske C, Reilly GC, Lacroix D and Perrault CM (2016) In Vitro Bone Cell Models: Impact of Fluid Shear Stress on Bone Formation. Front. Bioeng. Biotechnol. 4:87. doi: 10.3389/fbioe.2016.00087 
Figure 2: Effect of Wnt signaling pathway on bone mass regulation

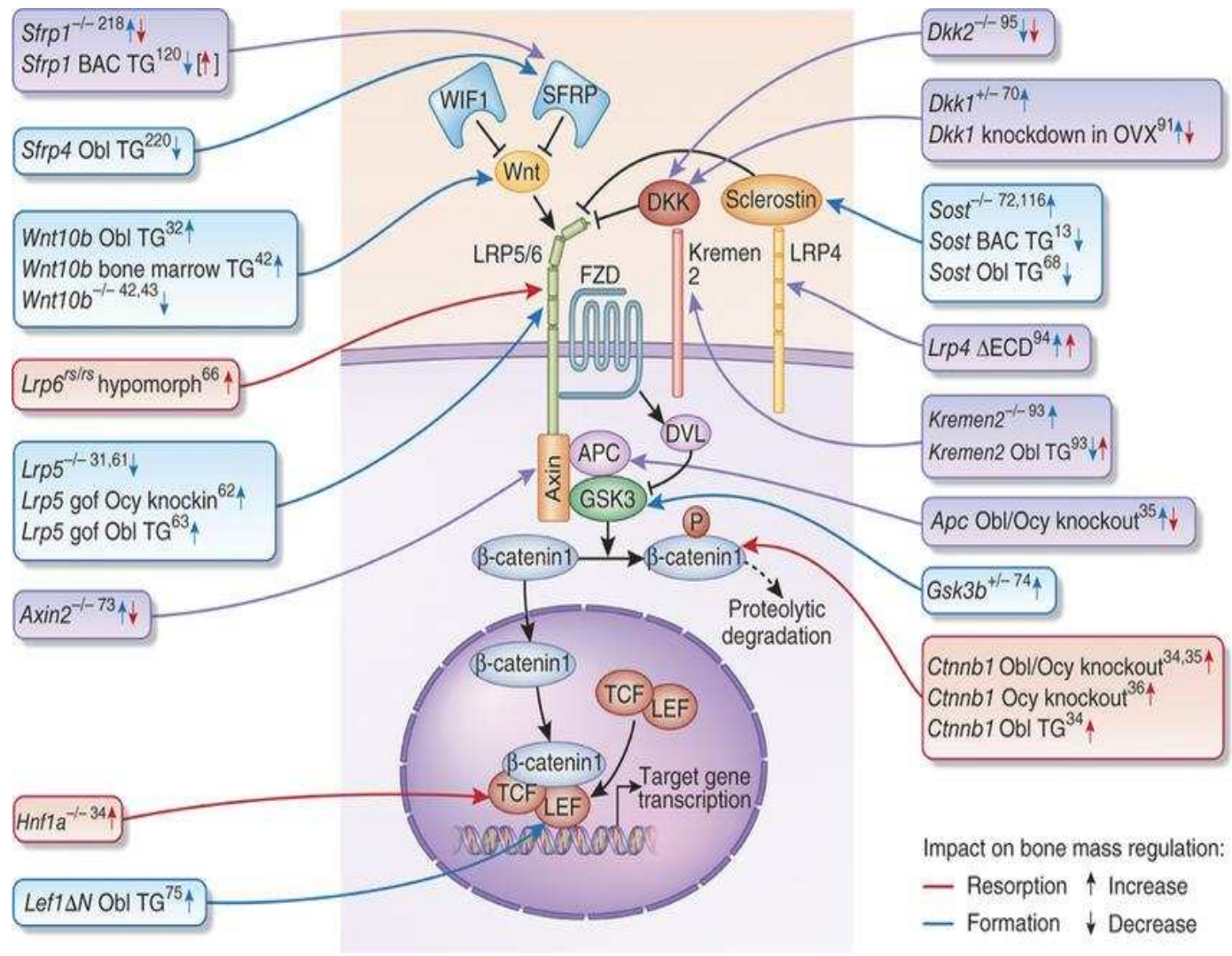

Adapted from: Baron R, Kneissel M. WNT signaling in bone homeostasis and disease: from human mutations to treatments. Nat Med. 2013 Feb;19(2):179-92. 
Figure 3: Interaction between $\mathrm{Wnt} / \beta$-catenin signaling and bone cells

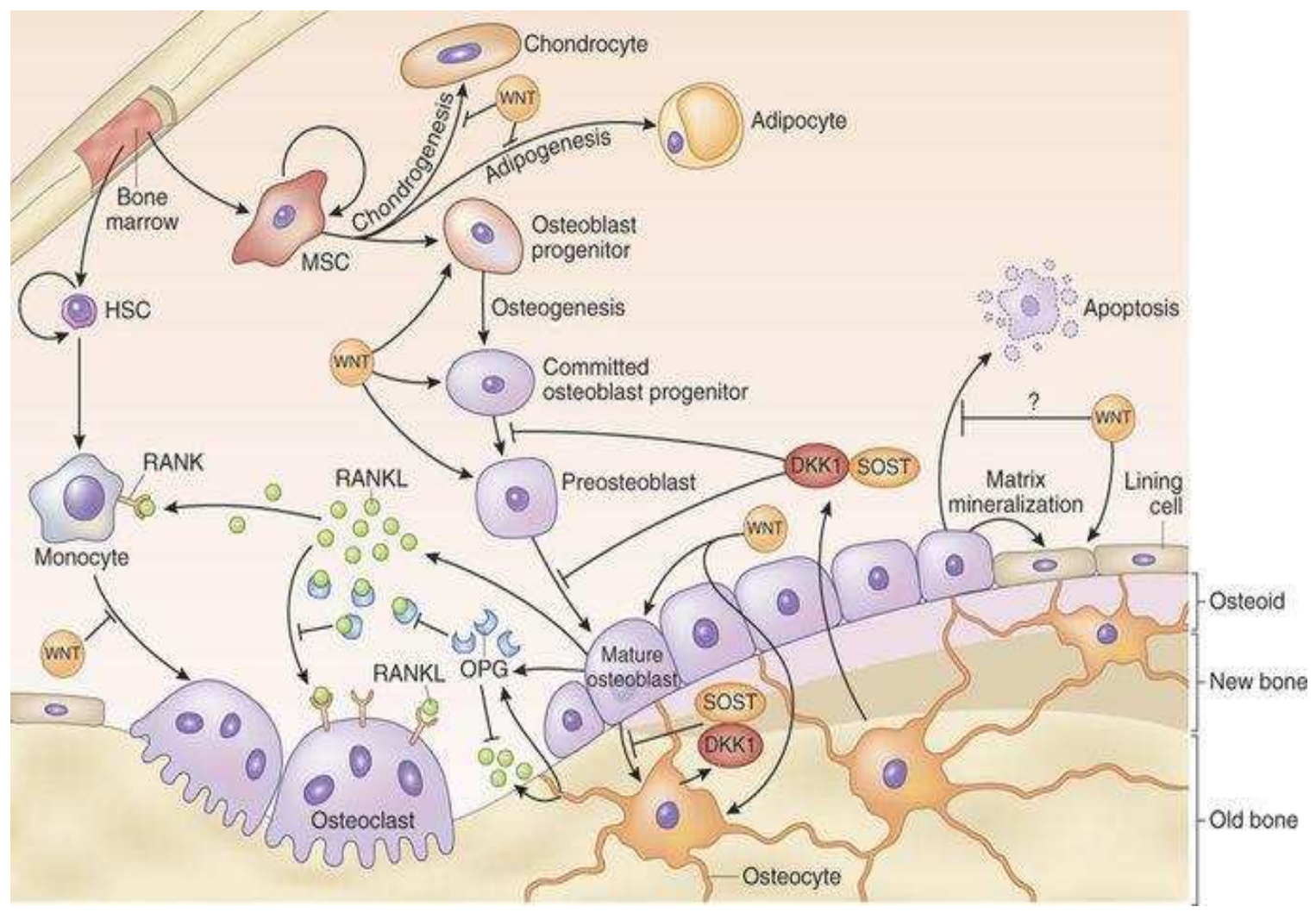

Adapted from: Baron R, Kneissel M. WNT signaling in bone homeostasis and disease: from human mutations to treatments. Nat Med. 2013 Feb;19(2):179-92. 
Figure 4: Overview of the Wnt signaling

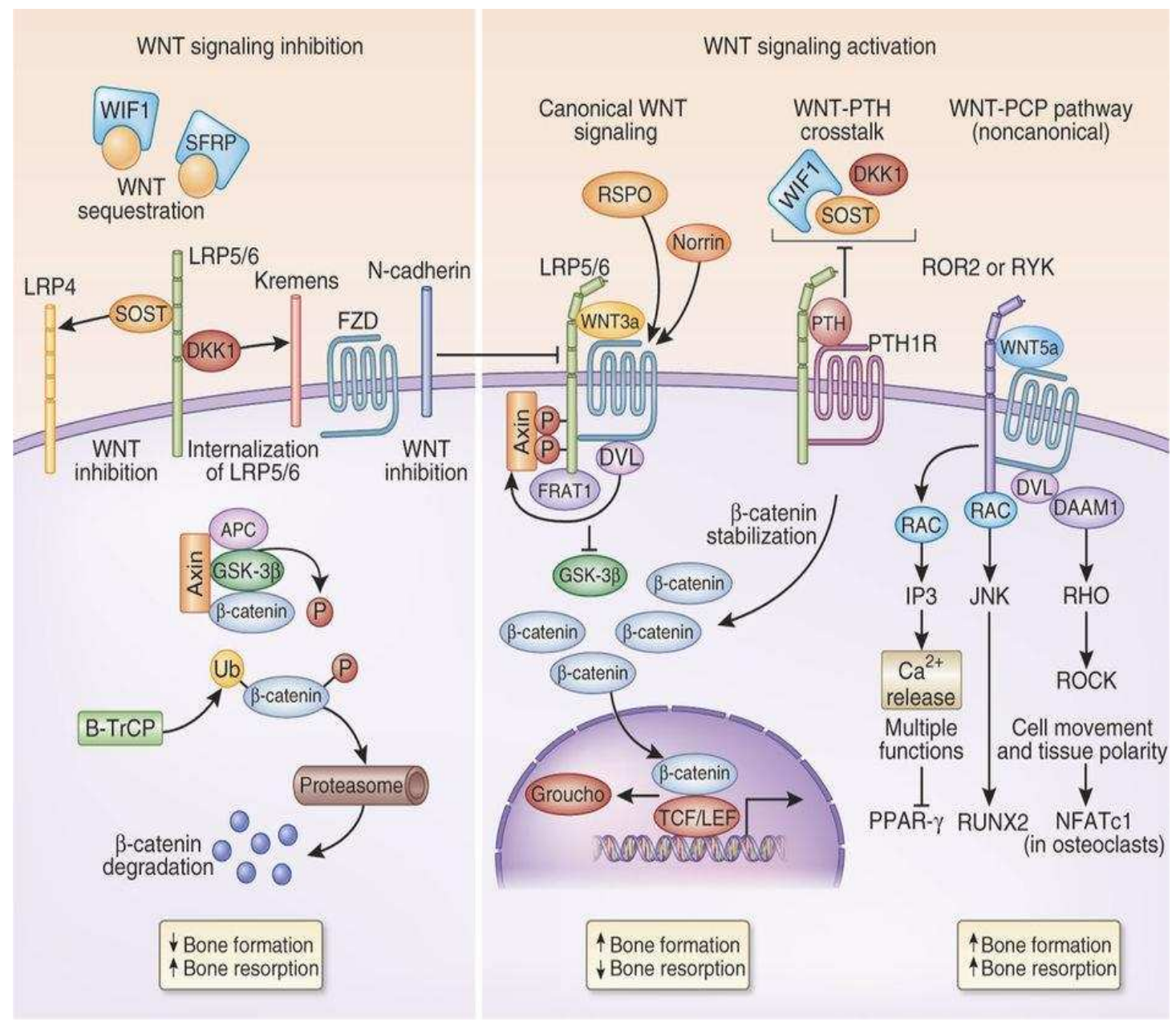

Adapted from: Baron R, Kneissel M. WNT signaling in bone homeostasis and disease: from human mutations to treatments. Nat Med. 2013 Feb;19(2):179-92. 
Figure 5: Regulation of secretion of the WNT inhibitor, SOST, from osteocytes inactivates the $\mathrm{Wnt} / \beta$-catenin signaling

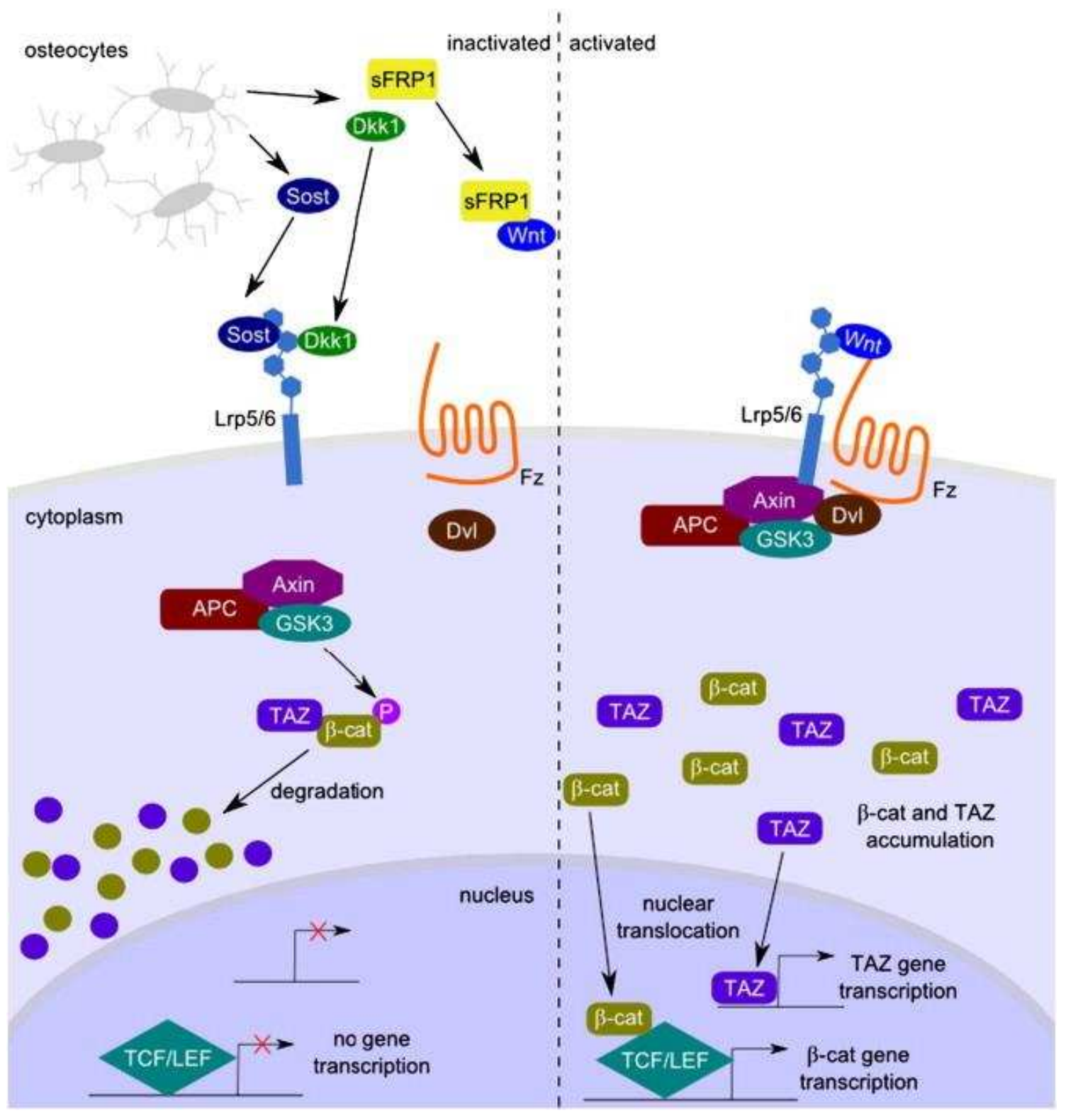

Adapted from: Burgers TA, Williams BO. Regulation of Wnt/ß-catenin signaling within and from osteocytes. Bone. 2013 Jun;54(2):244-9. 
Figure 6: A schematic diagram showing the role of SOST in $\mathrm{Wnt} / \mathrm{\beta}$-catenin and RANKL/RANK.

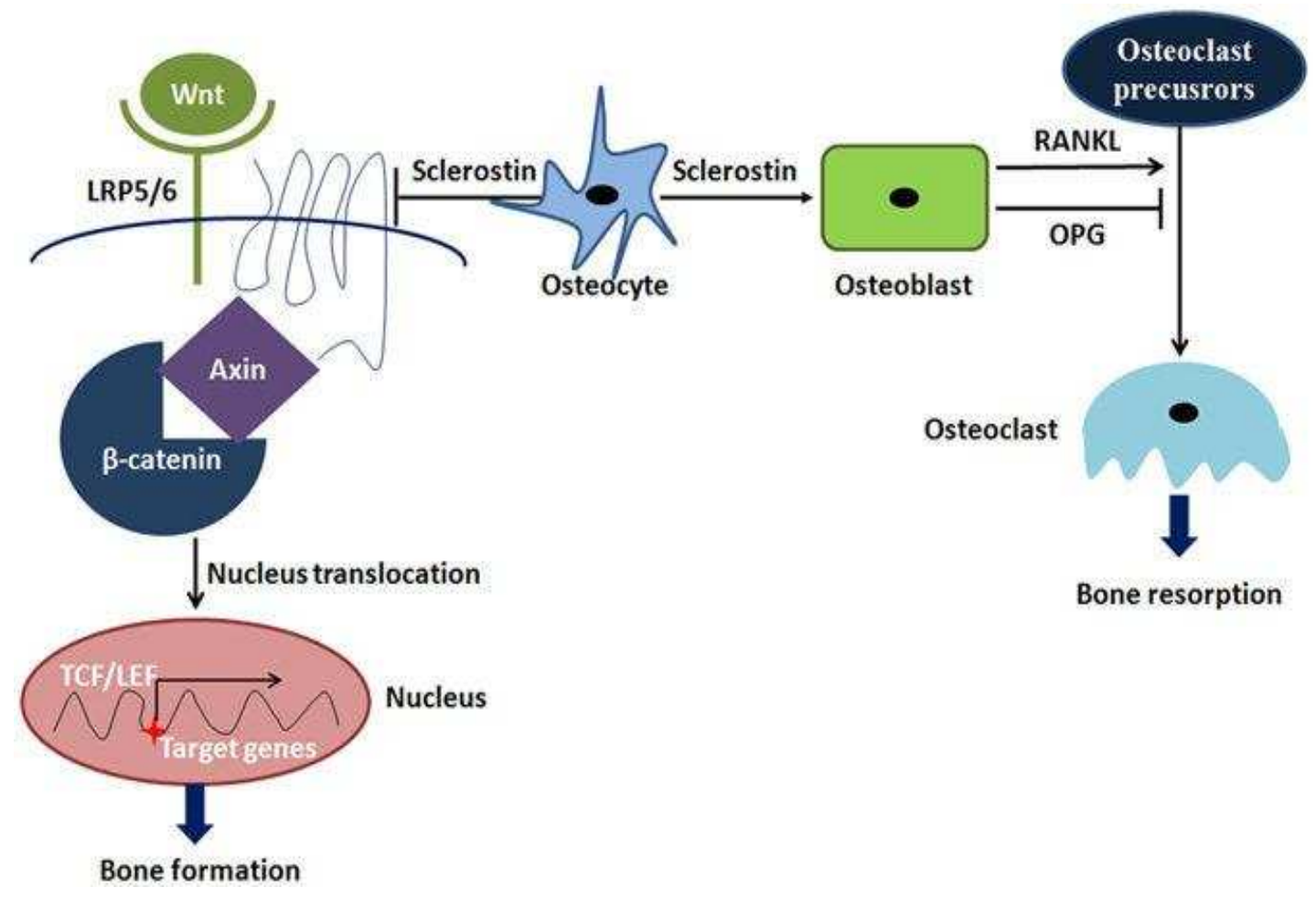

Adopted from: Diao X, Li Z, An B, Xin H, Wu Y, Li K, Feng F, Dou C. The Microdamage and Expression of Sclerostin in Peri-implant Bone under One-time Shock Force Generated by Impact. Sci Rep. 2017 Jul 26;7(1):6508. 
Figure 7: Wnt-5a can both activate and inhibit the canonical Wnt signaling depending on the receptor context

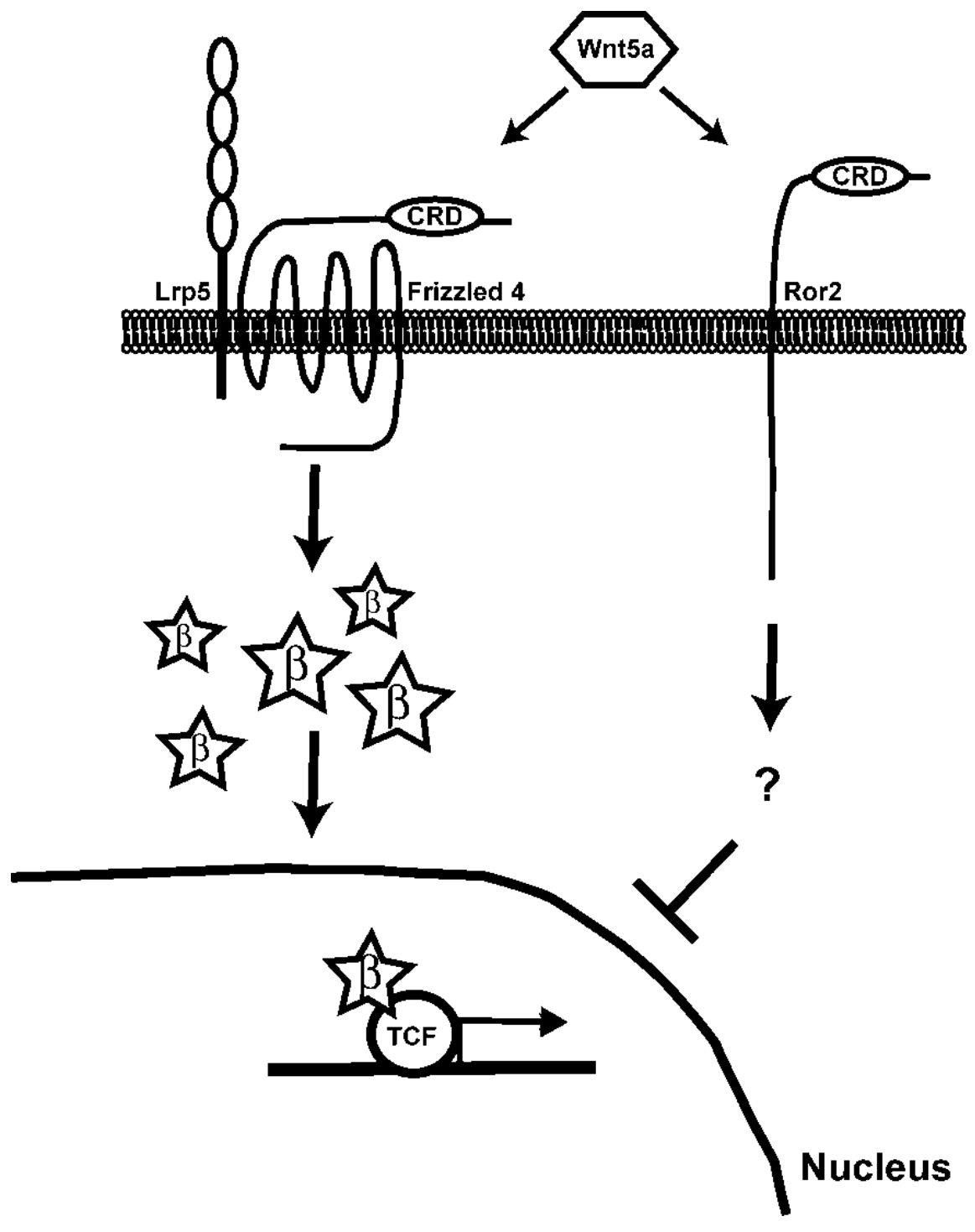

Adopted from: Leris A, Roberts T, Jiang W, Newbold R, Mokbel K. 2005. WNT-5A expression in human breast cancer. Anticancer Res 25:731-734. 
Figure 8: Distribution of the total amount of GCF SOST in the study groups. Dark lines show medians.

\section{Sclerostin (SOST)}

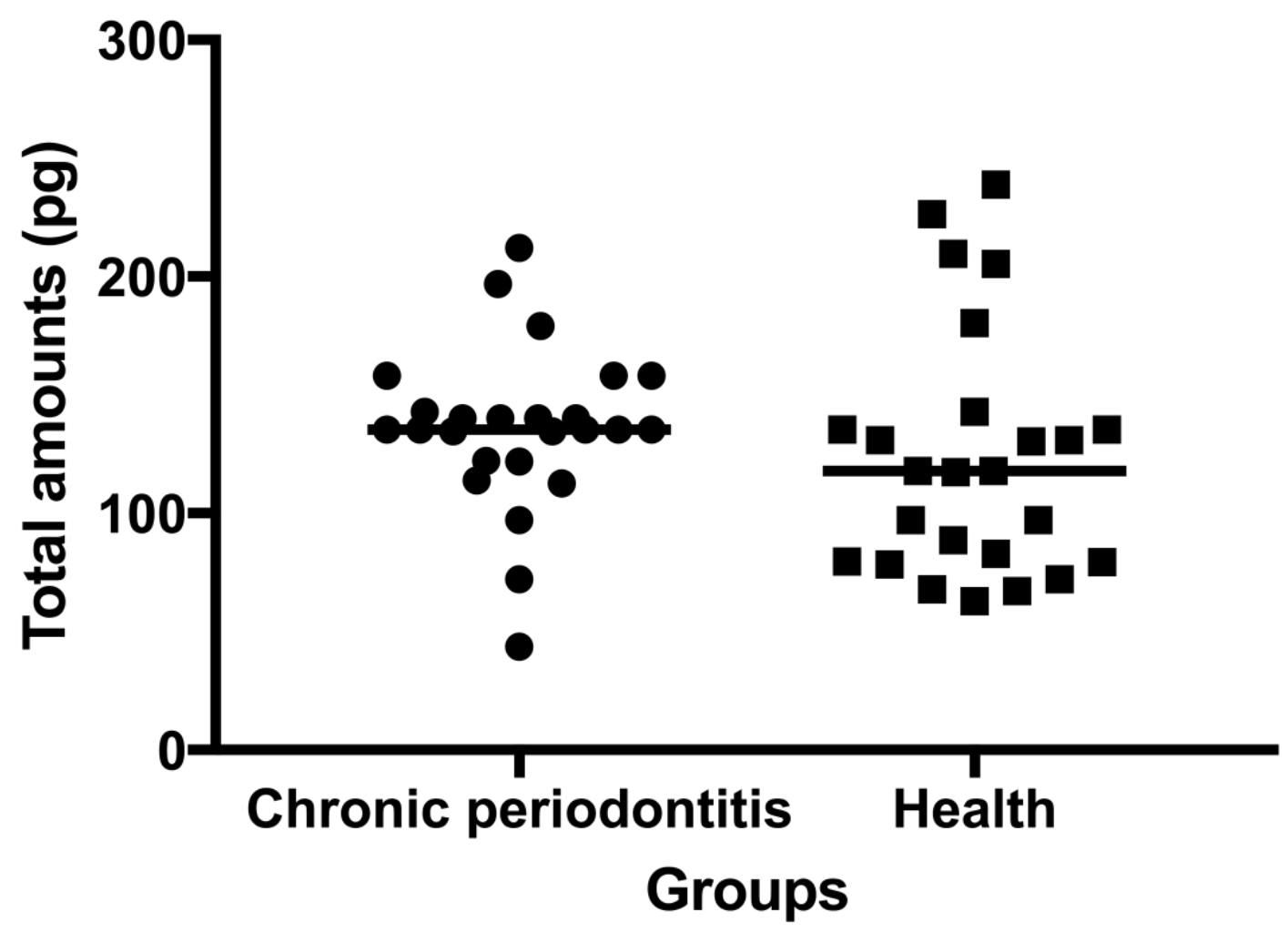

Mann-Whitney $U$ test was used to compare the protein total amounts of SOST between chronic periodontitis $(n=25)$ and periodontally healthy $(n=25)$ sites in chronic periodontitis and healthy patients, respectively. No significant differences were found between the two groups $(p=0.068)$. 
Figure 9: Distribution of the total amount of GCF Wnt-5a in the study groups. Dark lines show medians.

\section{Wnt-5a}

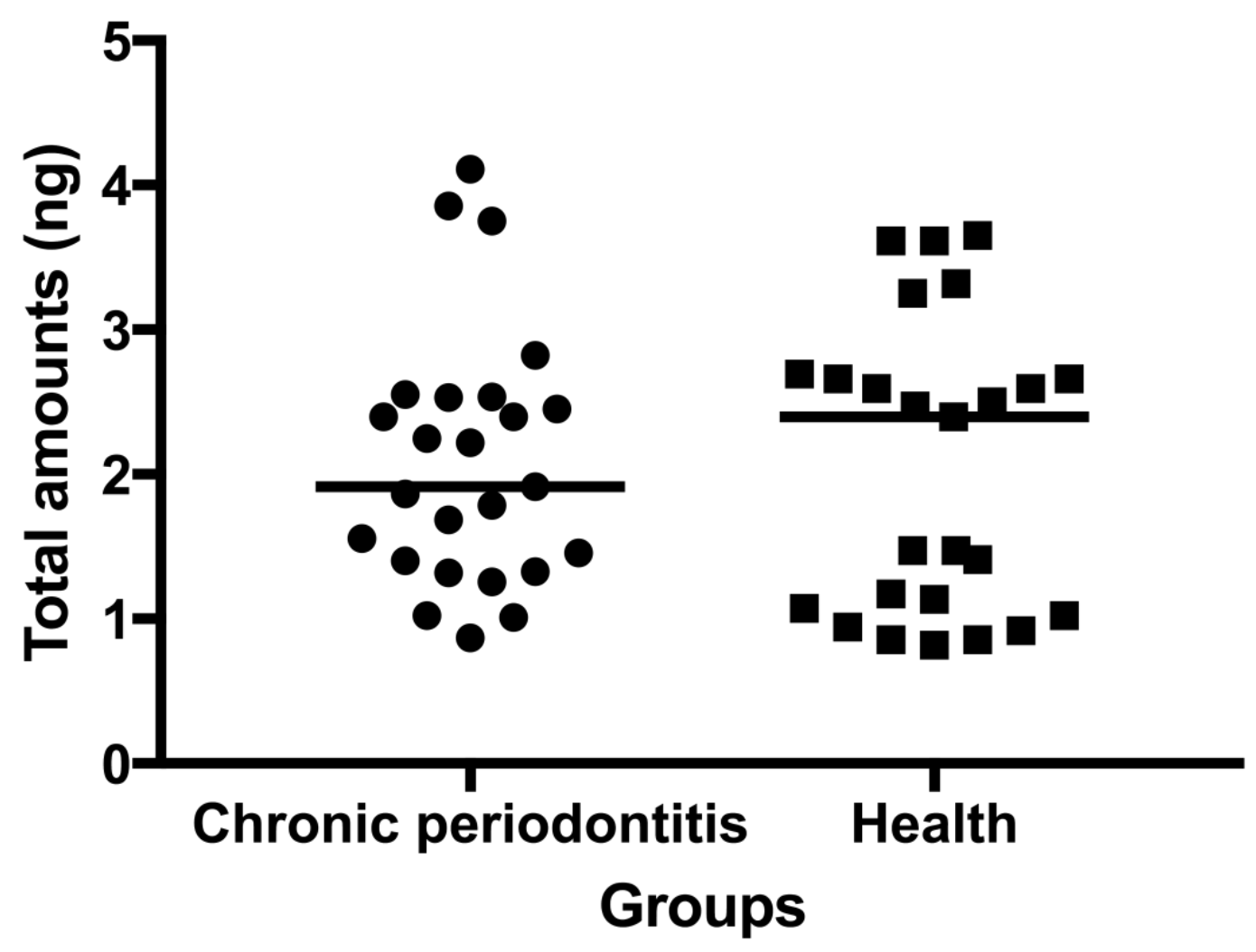

Mann-Whitney $\mathrm{U}$ test was used to compare the protein total amounts of Wnt-5a between chronic periodontitis $(n=25)$ and periodontally healthy $(n=25)$ sites in chronic periodontitis and healthy patients, respectively. No significant differences were found between the two groups $(p=0.854)$. 
Figure 10: Distribution of the total amount of GCF TNF- $\alpha$ in the study groups. Dark lines show medians.

\section{TNF- $\alpha$}

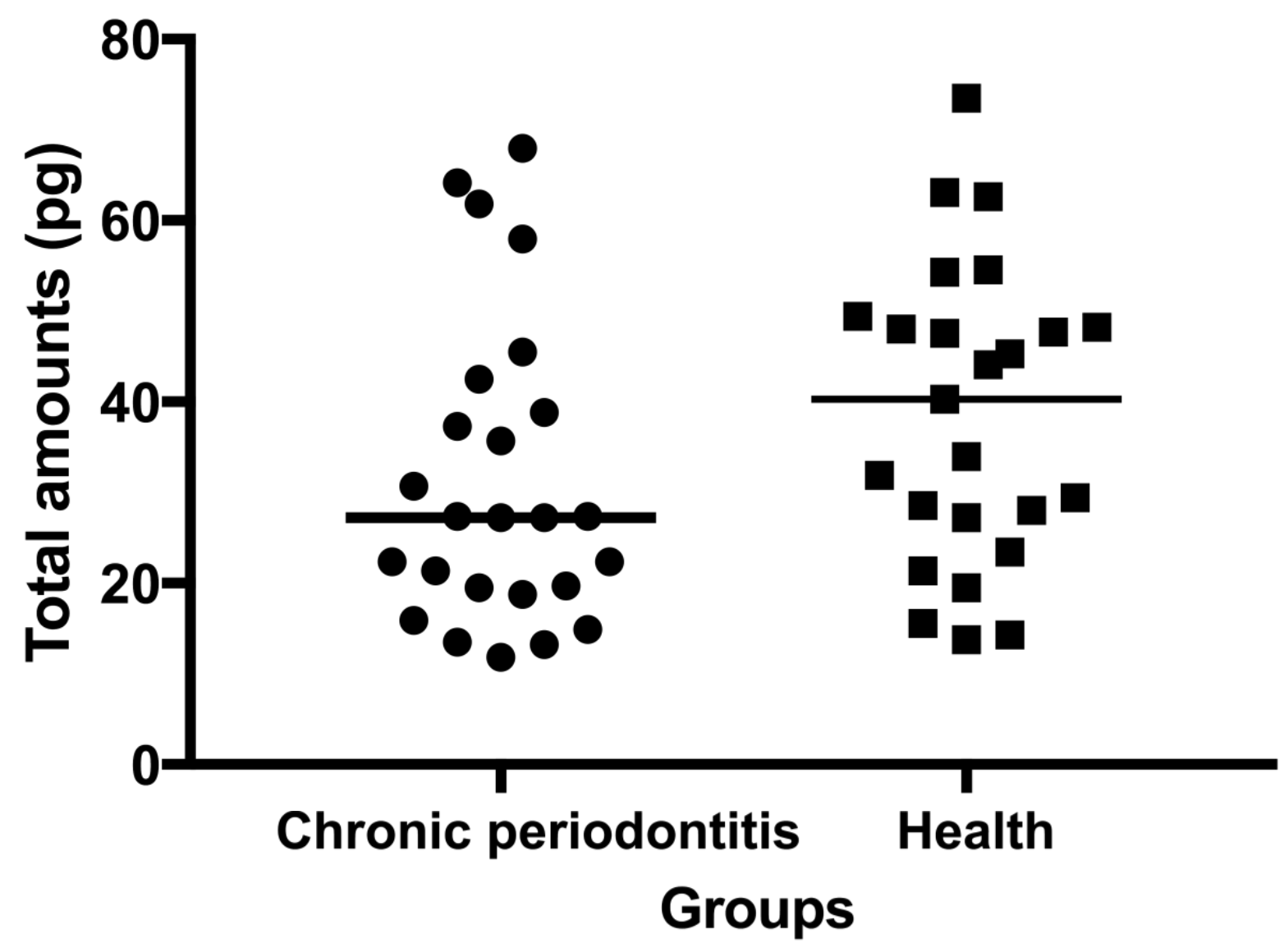

Mann-Whitney $U$ test was used to compare the protein total amounts of TNF- $\alpha$ between chronic periodontitis $(n=25)$ and periodontally healthy $(n=25)$ sites in chronic periodontitis and healthy patients, respectively. No significant differences were found between the two groups $(p=0.071)$. 
Figure 11a: Distribution of the concentration of GCF SOST in the study groups. Dark lines show medians.

\section{Sclerostin (SOST)}

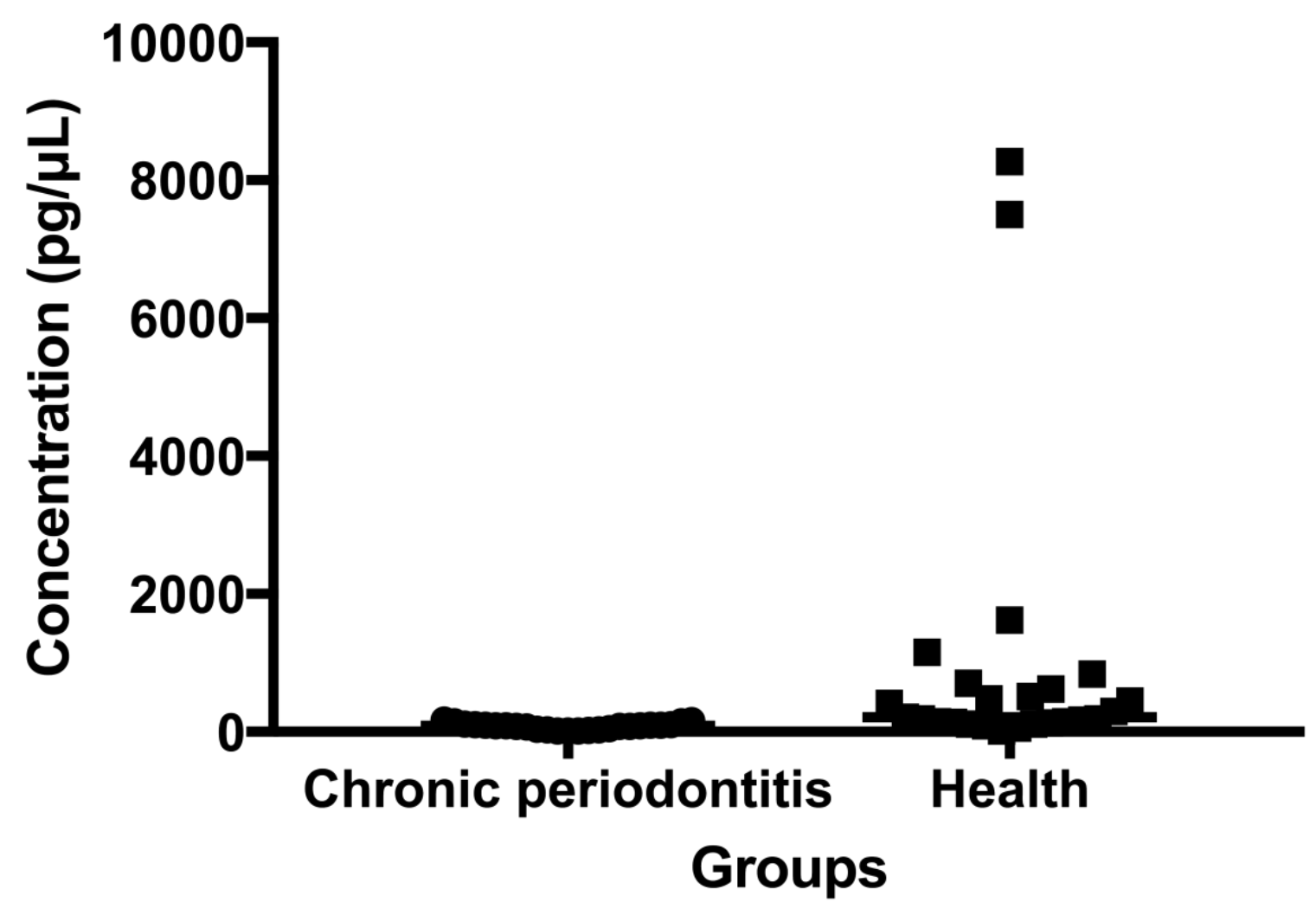

Mann-Whitney U test was used to compare the concentrations of SOST between chronic periodontitis $(n=25)$ and periodontally healthy $(n=25)$ sites in chronic periodontitis and healthy patients, respectively. Statistically significant differences were found between the two groups $(p<0.001)$. 
Figure 11b: Distribution of the concentration of GCF SOST in the study groups after removing two observational points (sampled sites) that are distant from the other observations. Dark lines show medians.

\section{Sclerostin (SOST)}

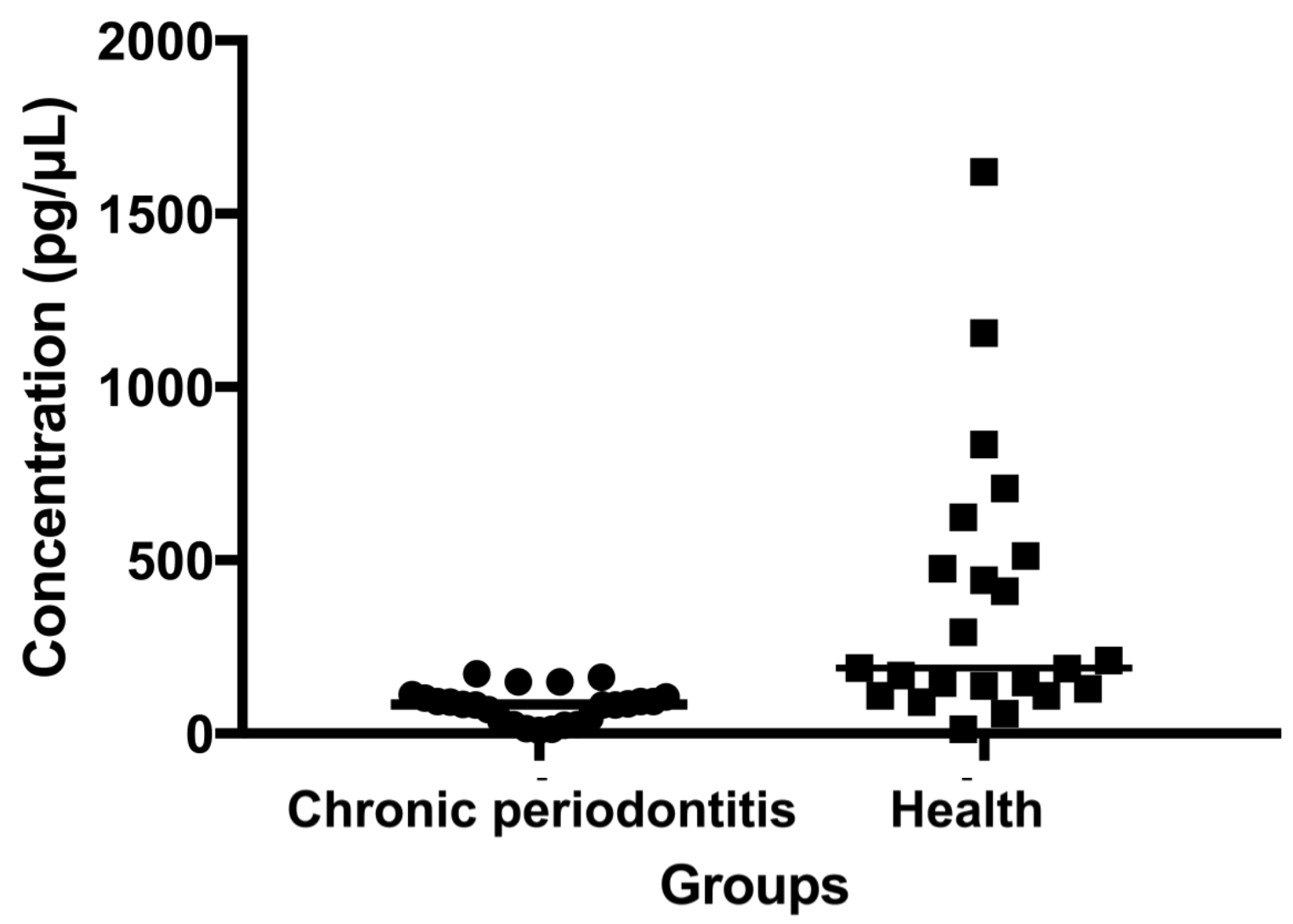


Figure 12: Distribution of the concentration of GCF Wnt-5a in the study groups. Dark lines show medians.

\section{Wnt-5a}

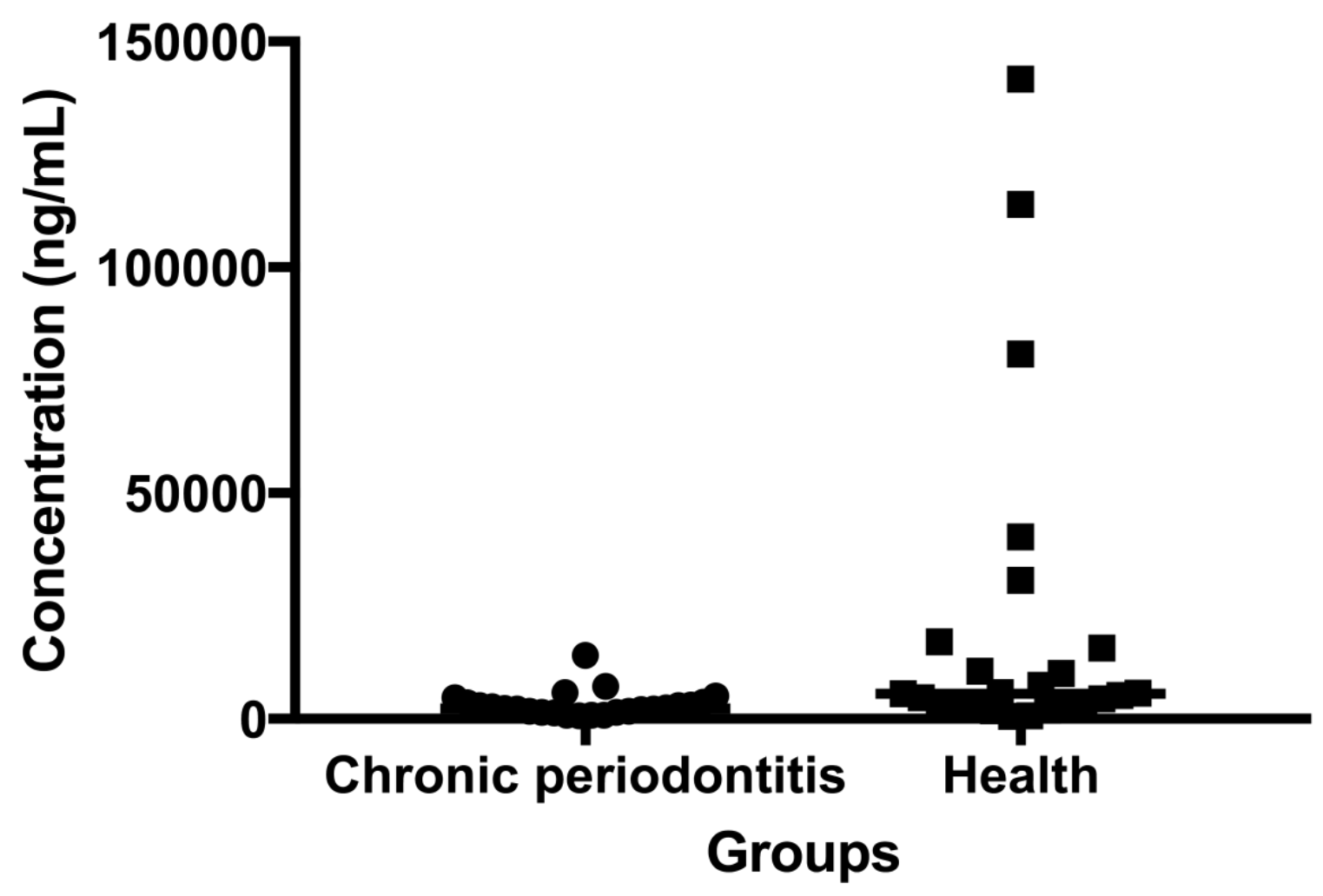

Mann-Whitney $U$ test was used to compare the concentrations of Wnt-5a between chronic periodontitis $(n=25)$ and periodontally healthy $(n=25)$ sites in chronic periodontitis and healthy patients, respectively. Statistically significant differences were found between the two groups $(p=0.003)$. 
Figure 13: Distribution of the concentrations of GCF TNF- $\alpha$ in the study groups. Dark lines show medians.

\section{TNF- $\alpha$}

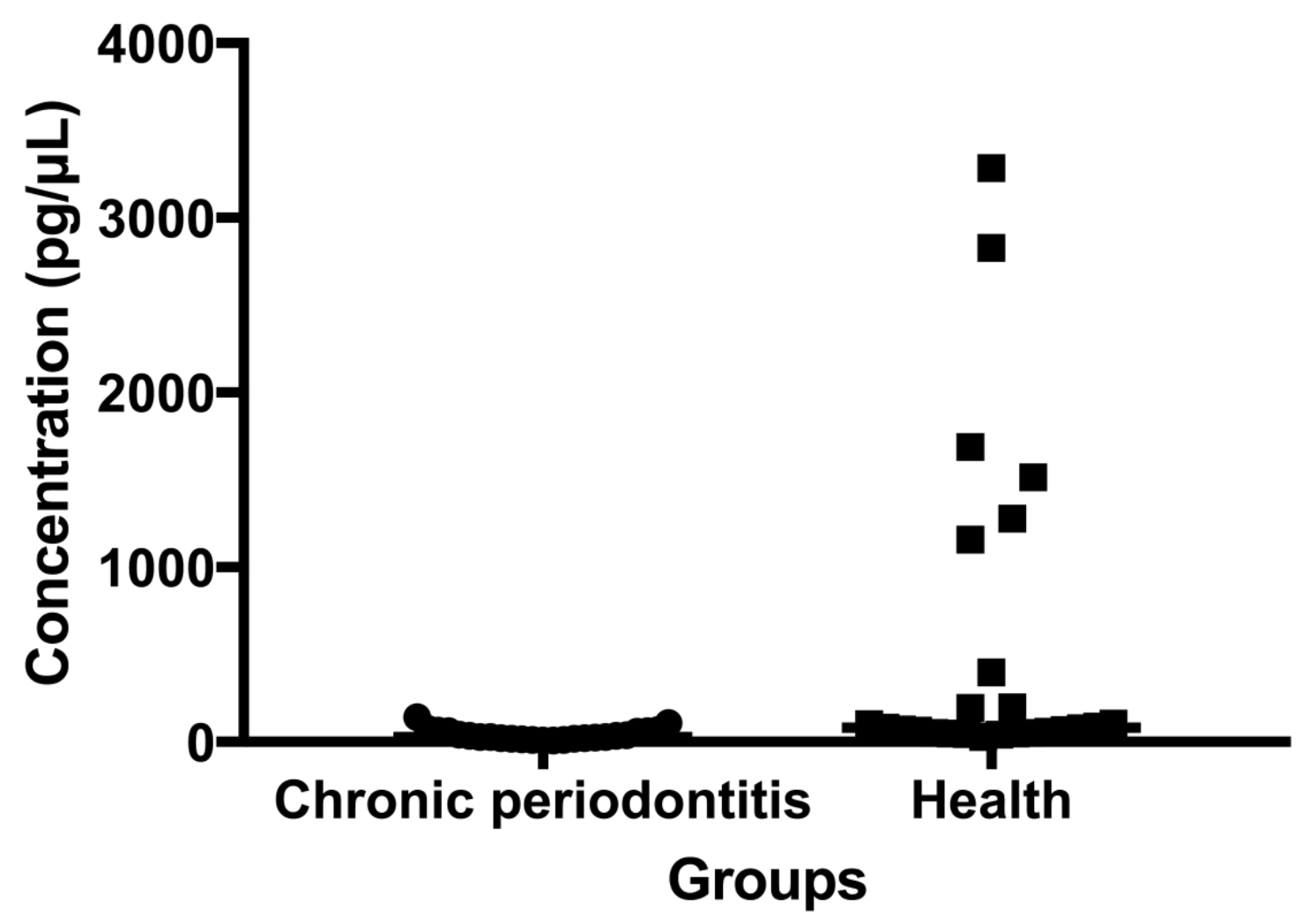

Mann-Whitney $U$ test was used to compare the concentrations of TNF- $\alpha$ between chronic periodontitis $(n=25)$ and periodontally healthy $(n=25)$ sites in chronic periodontitis and healthy patients, respectively. Statistically significant differences were found between the two groups $(p<0.001)$. 
Figure 14: Distribution of the total amounts of GCF SOST of the diseased and healthy sites in periodontitis patients. Dark lines show medians.

\section{Sclerostin (SOST)}

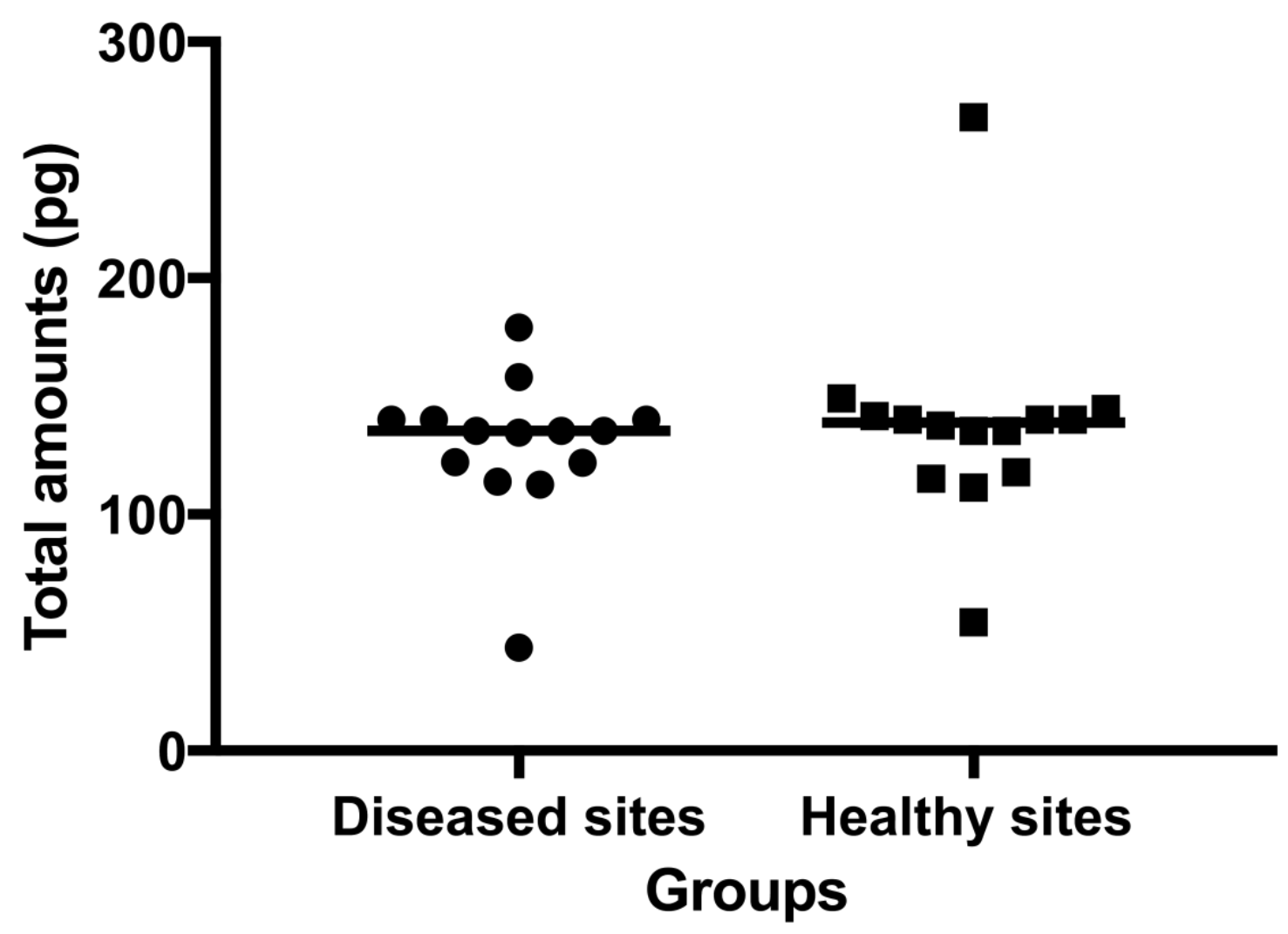

Wilcoxon signed-rank test was used to compare the protein total amounts of SOST between diseased sites and healthy sites in a subset of chronic periodontitis patients $(n=14)$ that exhibited both diseased and healthy sites. No significant differences were found between the two groups $(p=0.413)$. 
Figure 15: Distribution of the total amounts of GCF Wnt-5a of the diseased and healthy sites in periodontitis patients. Dark lines show medians.

\section{Wnt-5a}

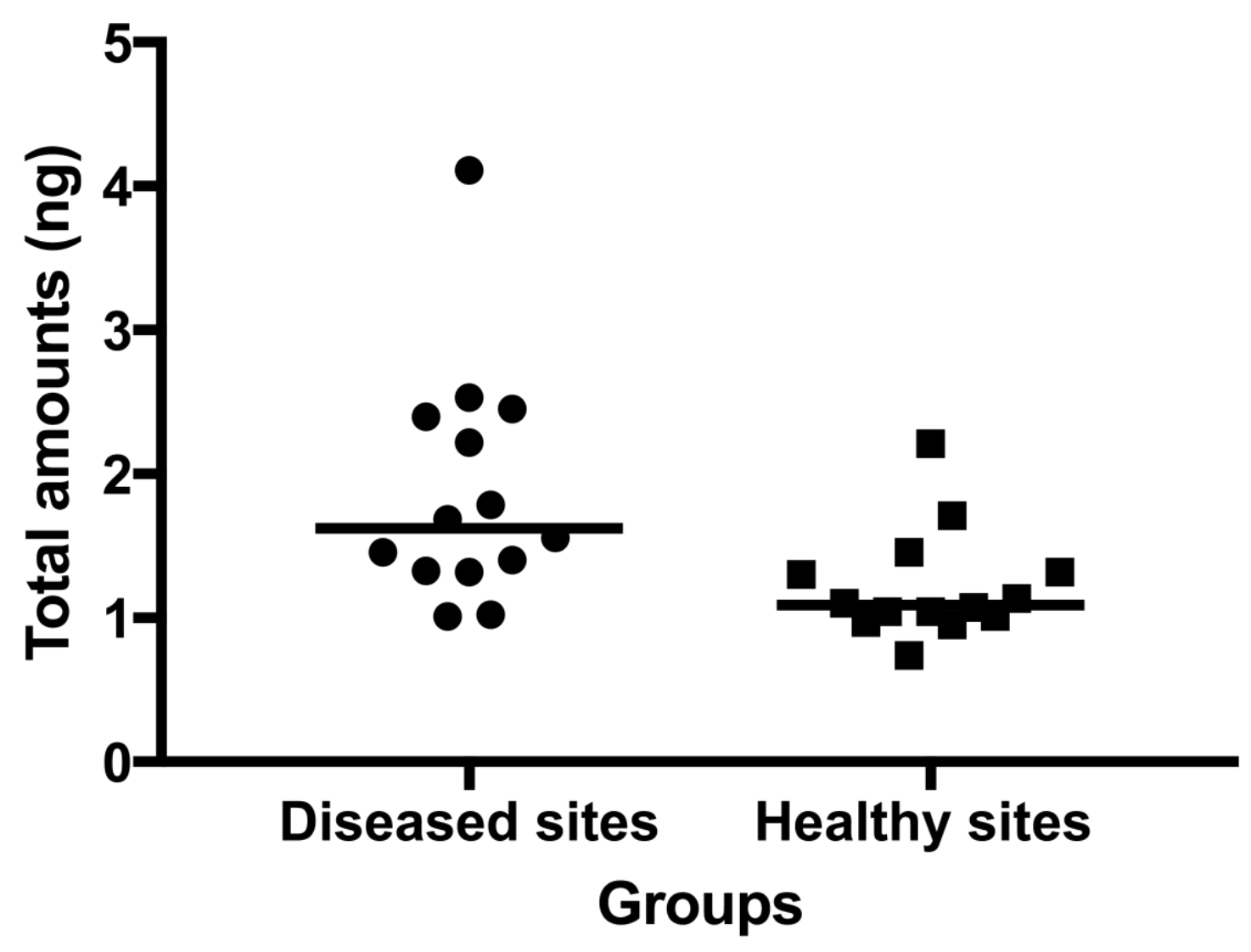

Wilcoxon signed-rank test was used to compare the protein total amounts of Wnt-5a between diseased sites and healthy sites in a subset of chronic periodontitis patients $(n=14)$ that exhibited both diseased and healthy sites. Statistically significant differences were found between the two groups $(p=0.017)$. 
Figure 16: Distribution of the total amounts of GCF TNF- $\alpha$ of the diseased and healthy sites in periodontitis patients. Dark lines show medians.

\section{TNF- $\alpha$}

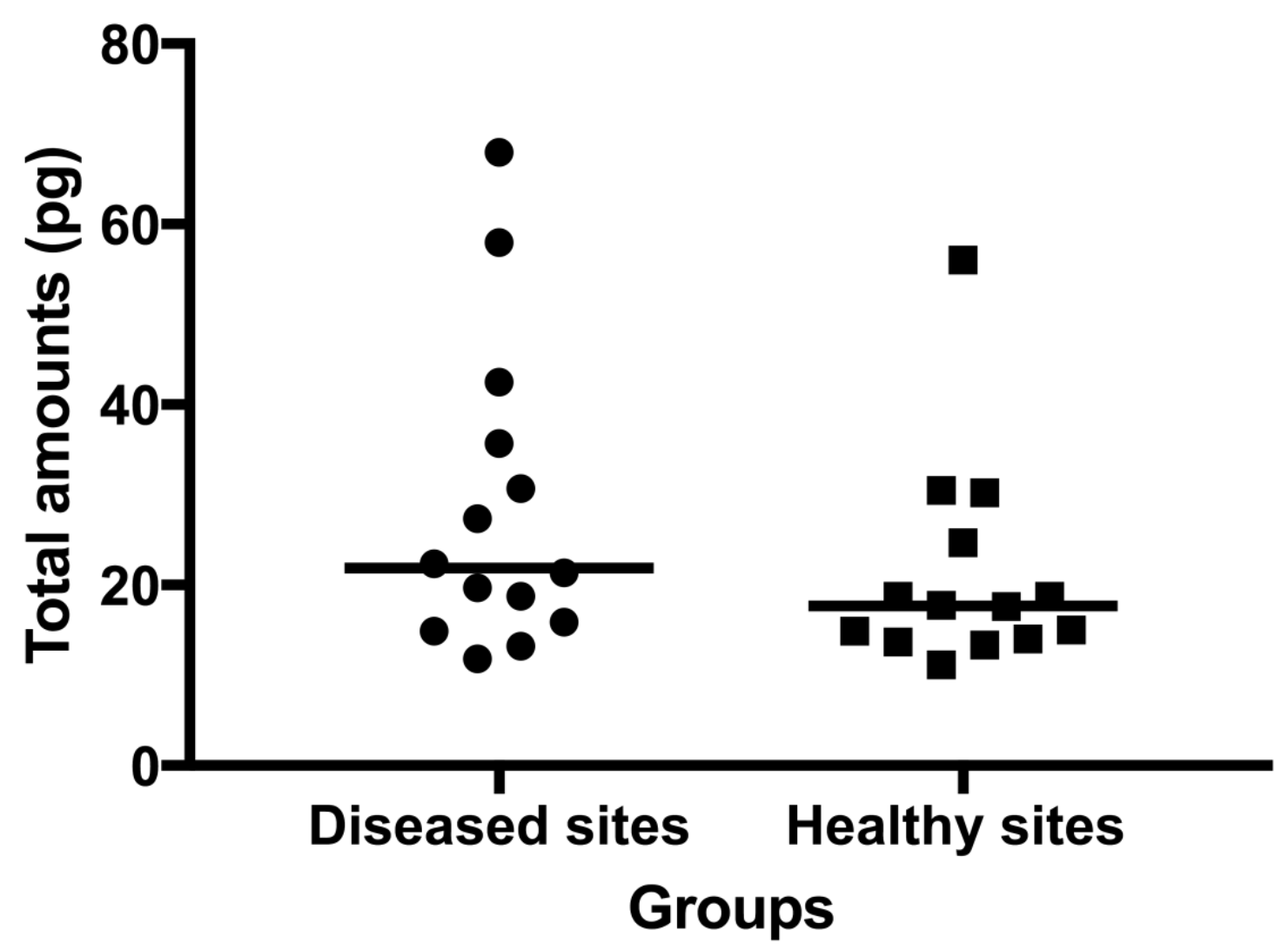

Wilcoxon signed-rank test was used to compare the protein total amounts of TNF- $\alpha$ between diseased sites and healthy sites in a subset of chronic periodontitis patients $(n=14)$ that exhibited both diseased and healthy sites. No statistically significant differences were found between the two groups $(p=0.194)$. 
Figure 17a: Distribution of the concentrations of GCF SOST of the diseased and healthy sites in periodontitis patients. Dark lines show medians.

\section{Sclerostin (SOST)}

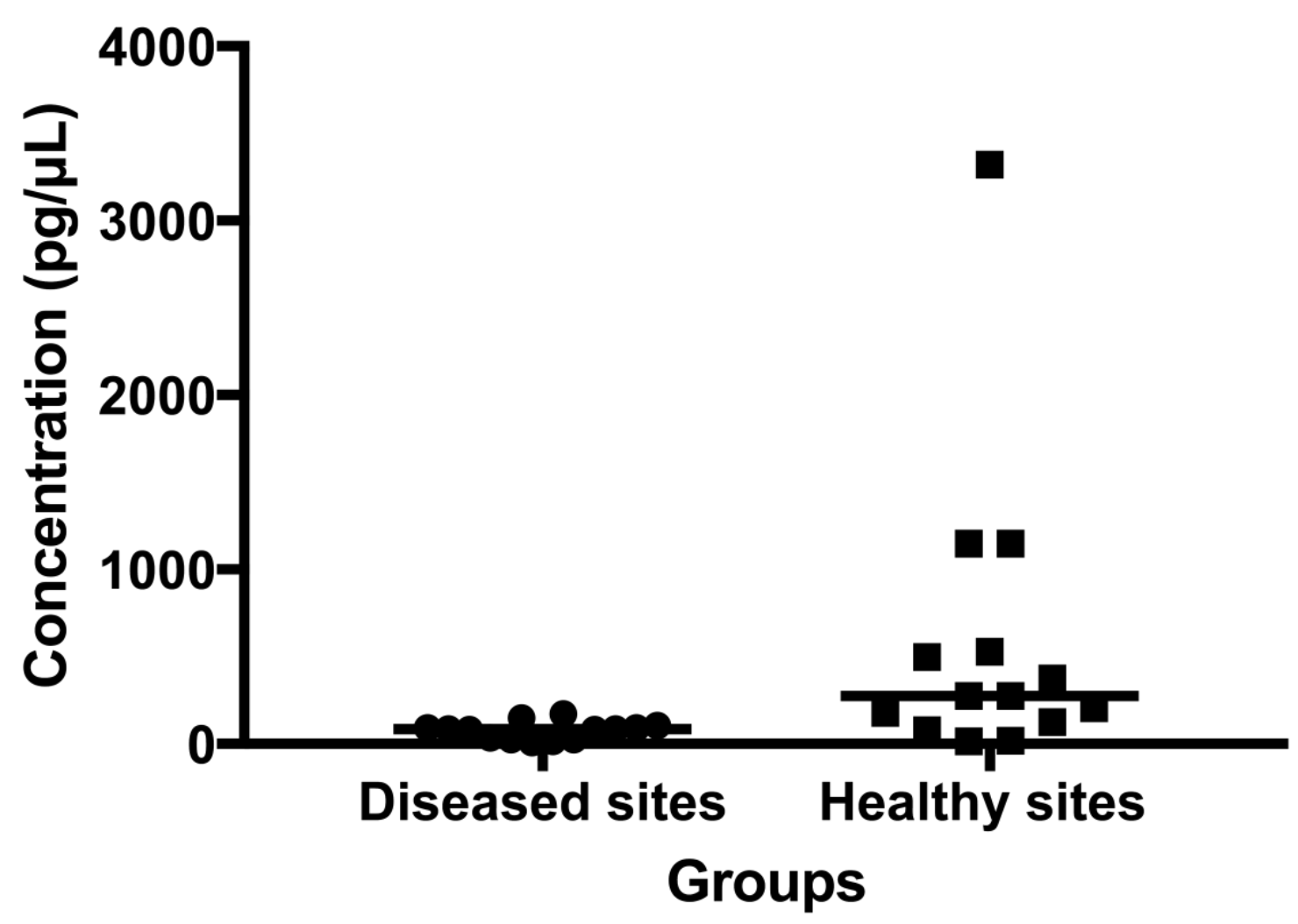

Wilcoxon signed-rank test was used to compare the concentrations of SOST between diseased sites and healthy sites in a subset of chronic periodontitis patients $(n=14)$ that exhibited both diseased and healthy sites. Statistically significant differences were found between the two groups $(p=0.001)$. 
Figure 17b: Distribution of the concentrations of GCF SOST of the diseased and healthy sites in periodontitis patients after removing an observational point (sampled site) that is distant from the other observations. Dark lines show medians.

\section{Sclerostin (SOST)}

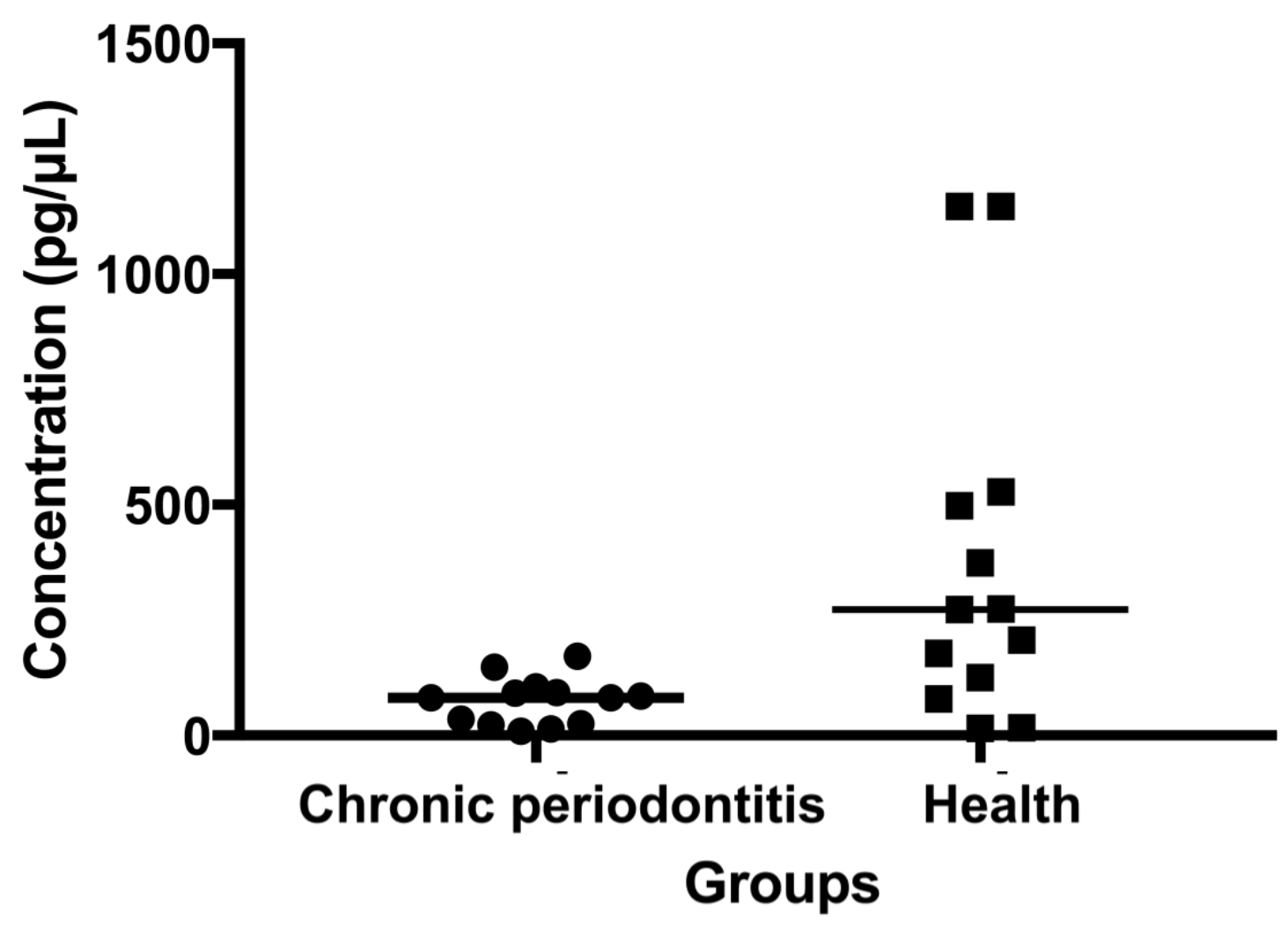


Figure 18a: Distribution of the concentrations of GCF Wnt-5a of the diseased and healthy sites in periodontitis patients. Dark lines show medians.

\section{Wnt-5a}

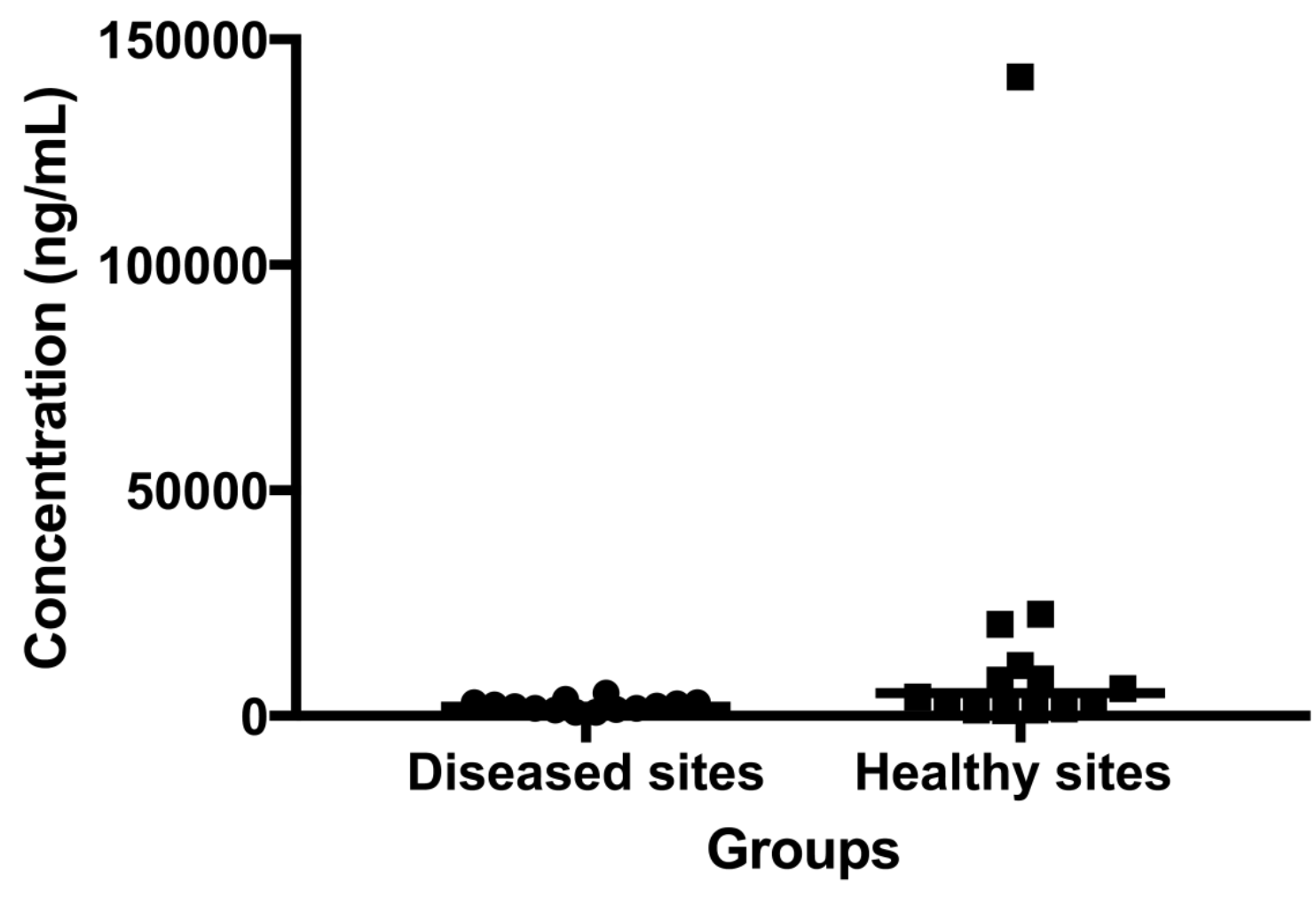

Wilcoxon signed-rank test was used to compare the concentrations of Wnt-5a between diseased sites and healthy sites in a subset of chronic periodontitis patients $(n=14)$ that exhibited both diseased and healthy sites. Statistically significant differences were found between the two groups $(p=0.001)$. 
Figure 18b: Distribution of the concentrations of GCF Wnt-5a of the diseased and healthy sites in periodontitis patients after removing an observational point (sampled site) that is distant from the other observations. Dark lines show medians.

\section{Wnt-5a}

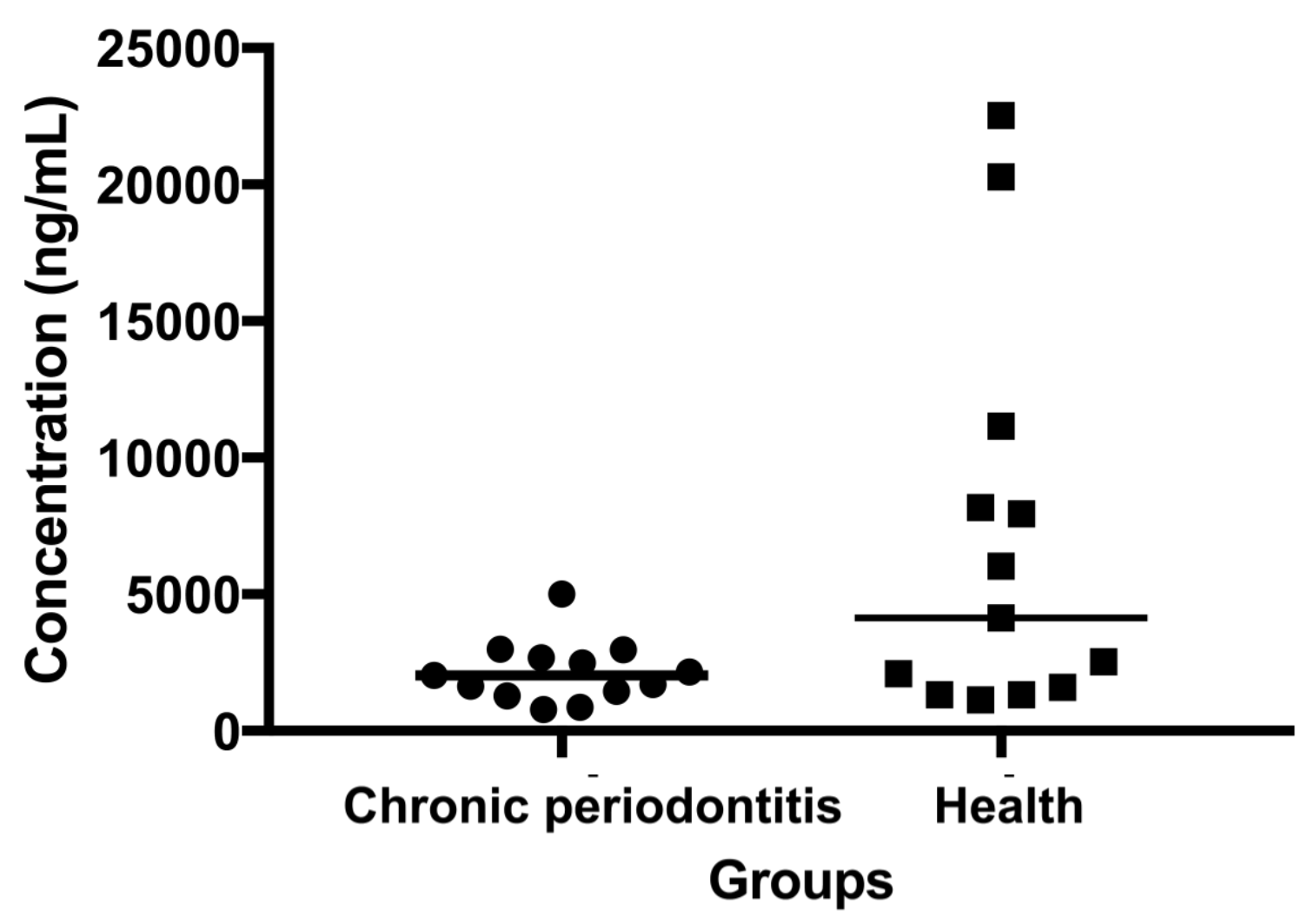


Figure 19: Distribution of the concentrations of GCF TNF- $\alpha$ of the diseased and healthy sites in periodontitis patients. Dark lines show medians.

\section{TNF- $\alpha$}

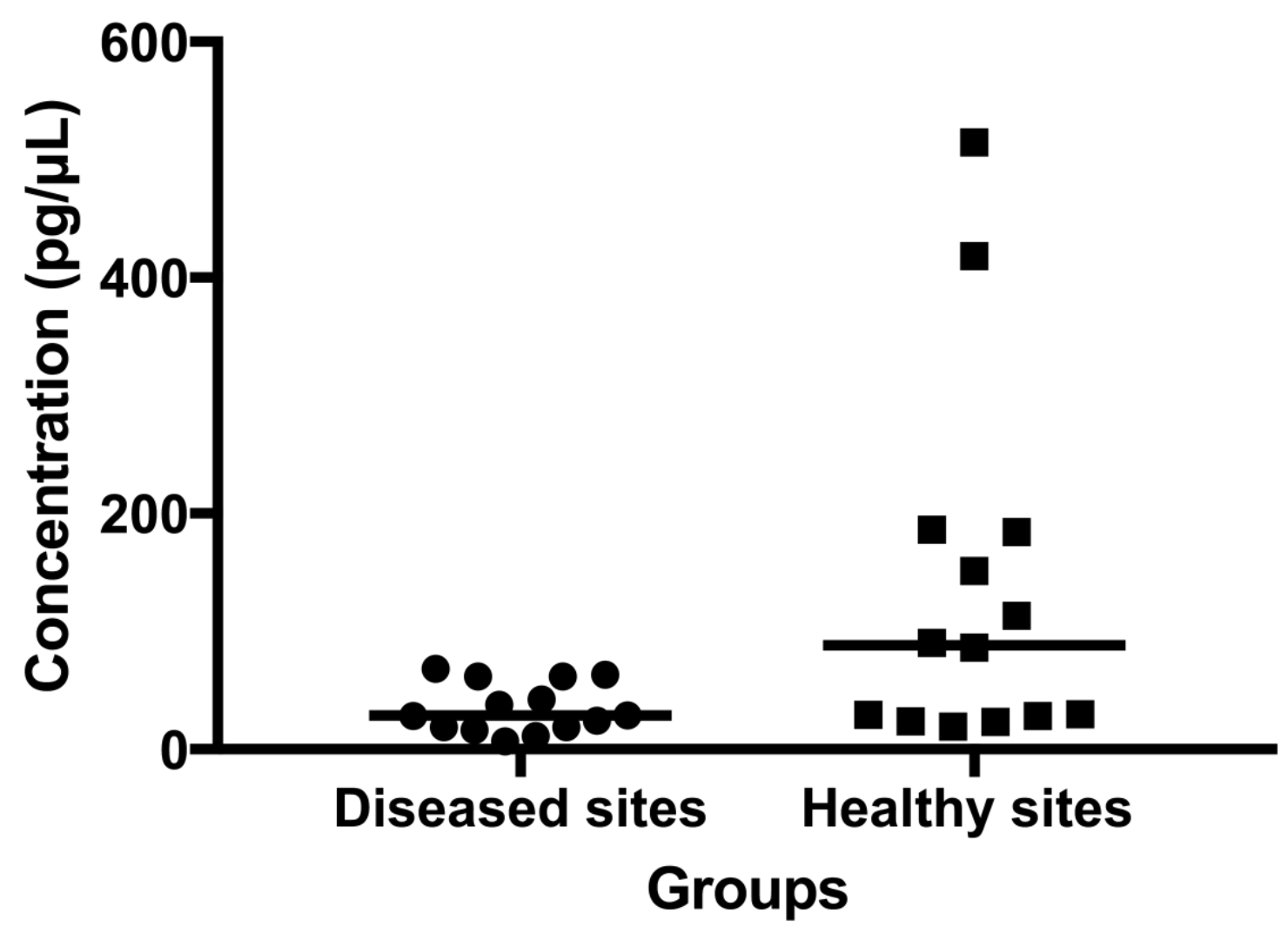

Wilcoxon signed-rank test was used to compare the concentrations of TNF- $\alpha$ between diseased sites and healthy sites in a subset of chronic periodontitis patients $(n=14)$ that exhibited both diseased and healthy sites. Statistically significant differences were found between the two groups $(p=0.001)$. 
Figure 20: ROC analysis of total amounts of GCF SOST to diagnose chronic periodontitis.

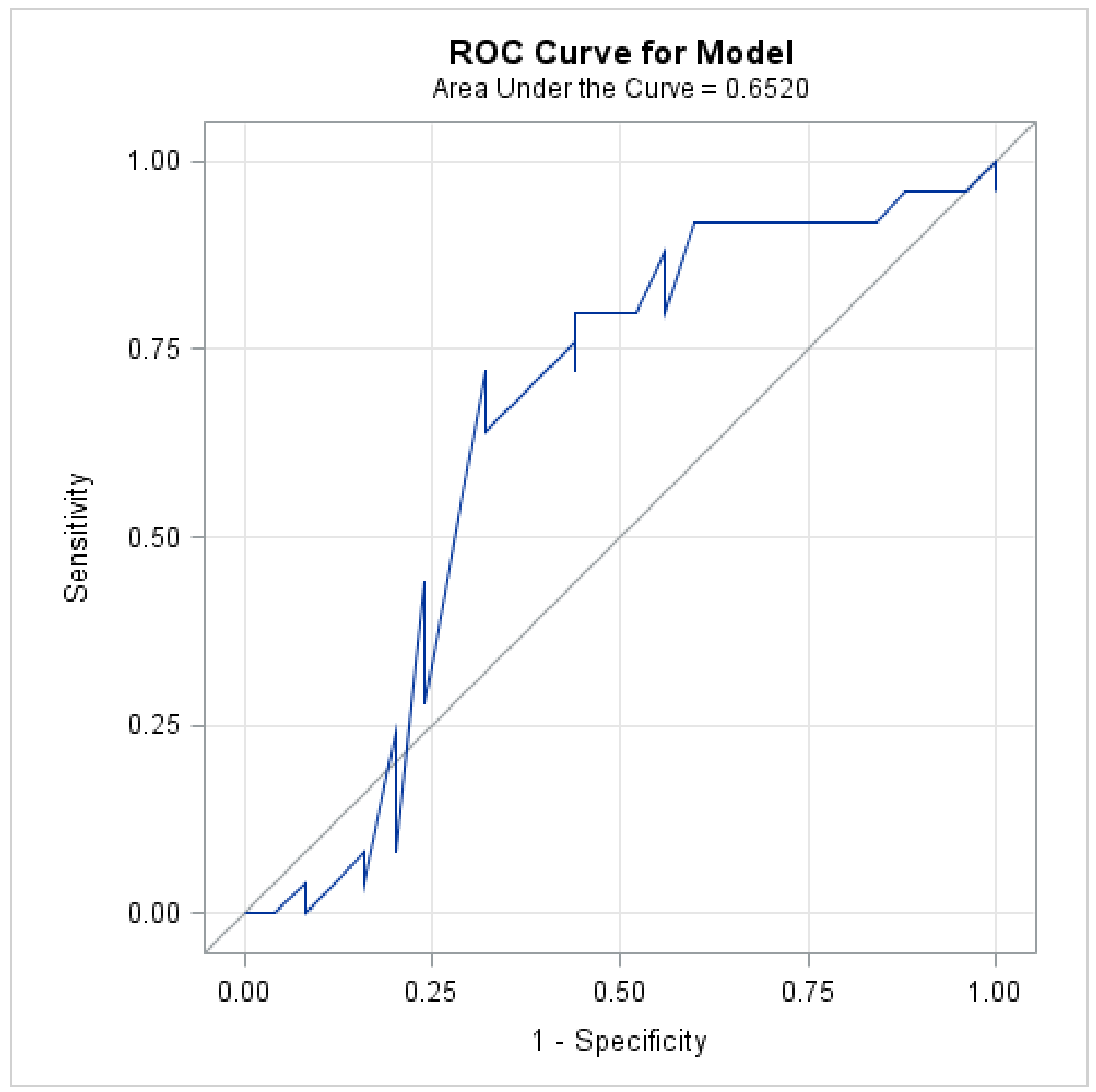


Figure 21: ROC analysis of total amounts of GCF Wnt-5a to diagnose chronic periodontitis.

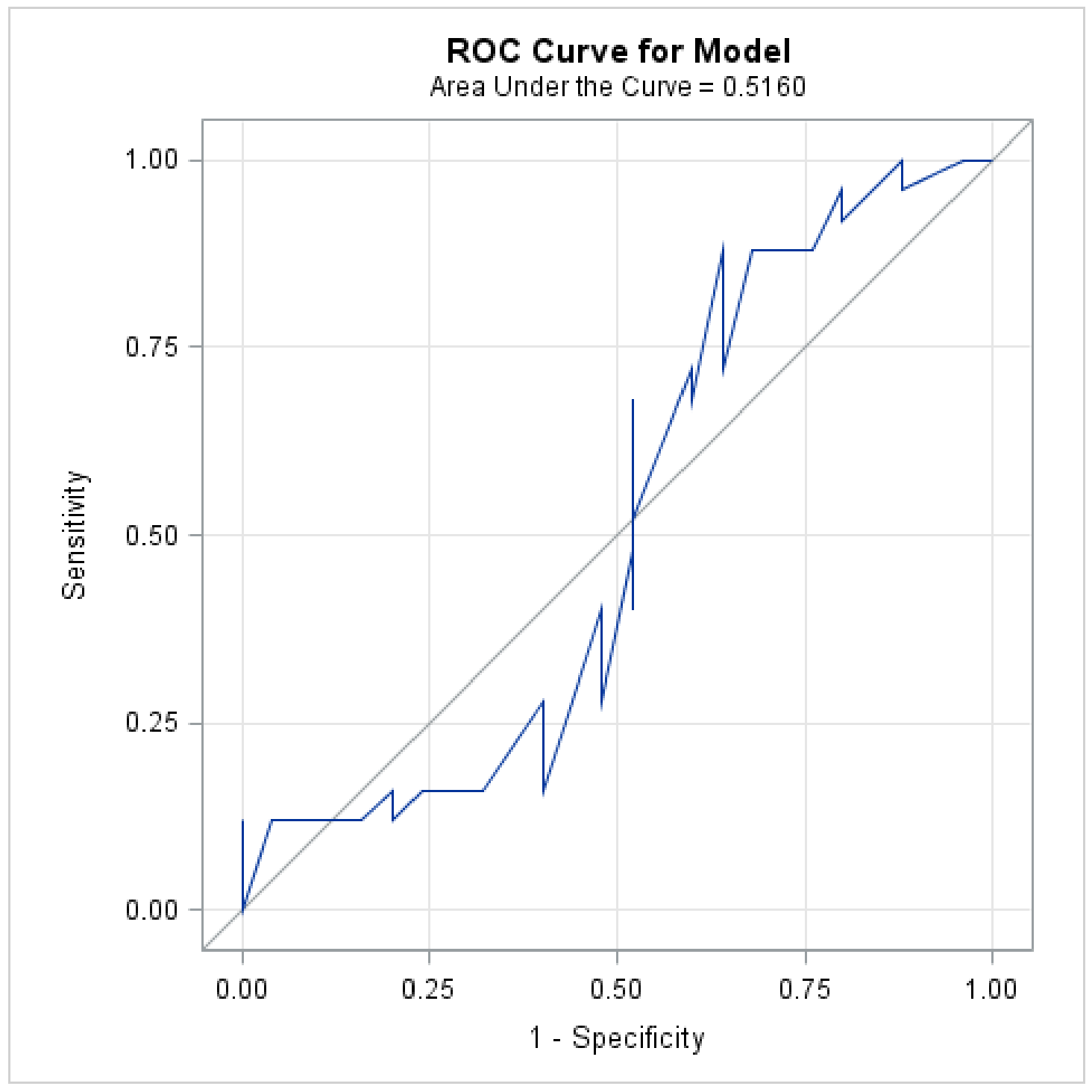


Figure 22: ROC analysis of total amounts of GCF TNF- $\alpha$ to diagnose chronic periodontitis.

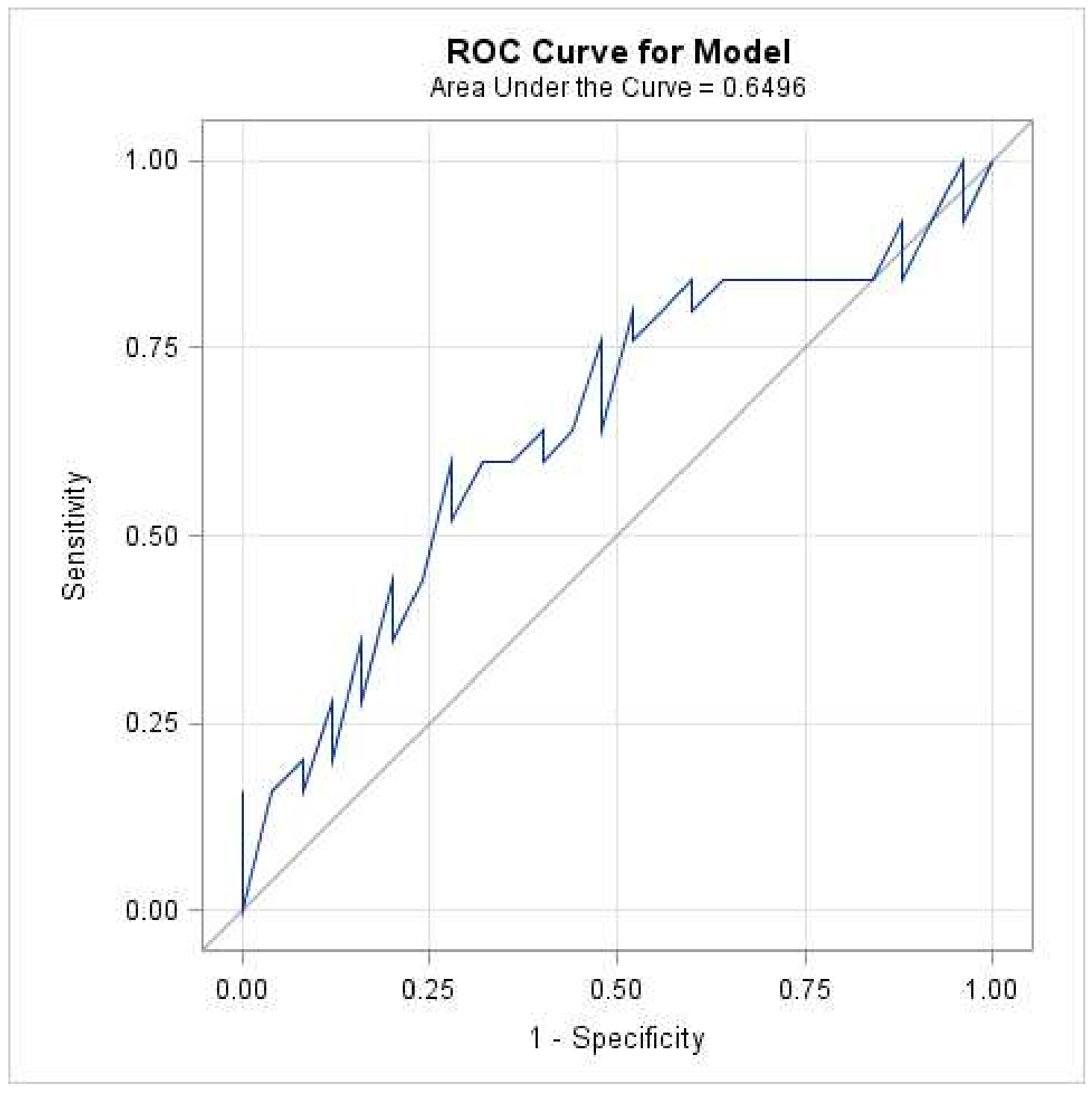


Figure 23: Distribution of the total amounts of GCF SOST of the diseased and healthy sites in the strictly diagnosed periodontitis and healthy patients. Dark lines show medians.

\section{Sclerostin (SOST)}

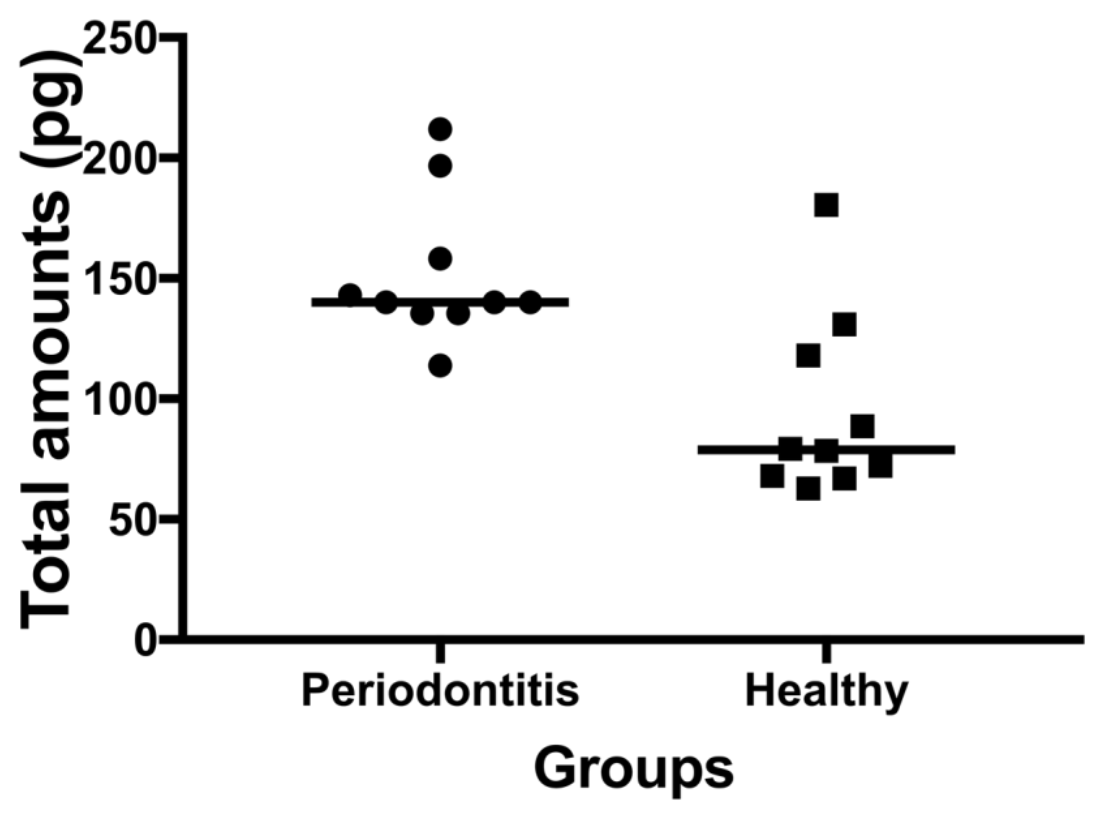

Mann-Whitney $U$ test was used to compare the total amounts of SOST between chronic periodontitis $(n=10)$ and periodontally healthy $(n=10)$ sites in strictly diagnosed chronic periodontitis and healthy patients, respectively. Statistically significant differences were found between the two study subgroups $(p=0.002)$. 
Figure 24: Distribution of the total amounts of GCF Wnt-5a of the diseased and healthy sites in the strictly diagnosed periodontitis and healthy patients. Dark lines show medians.

\section{WNT-5a}

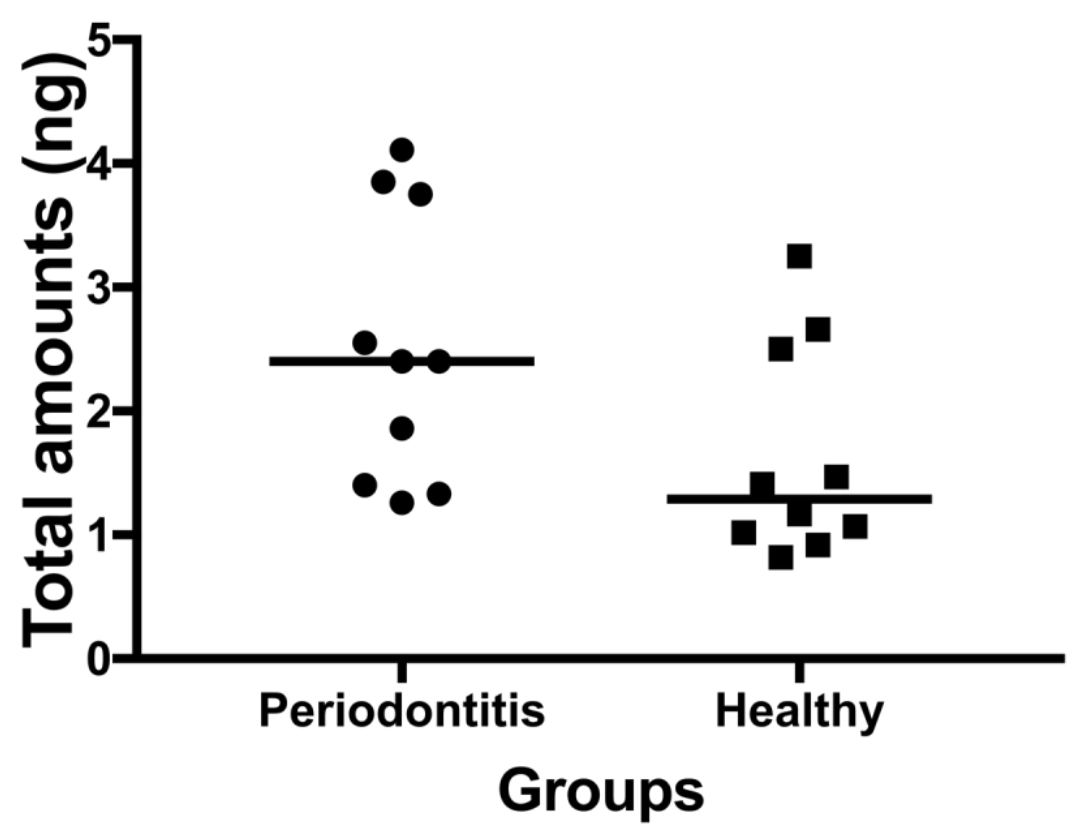

Mann-Whitney $\mathrm{U}$ test was used to compare the total amounts of Wnt-5a between chronic periodontitis $(n=10)$ and periodontally healthy $(n=10)$ sites in strictly diagnosed chronic periodontitis and healthy patients, respectively. No significant differences were found between the two study subgroups $(p=0.070)$. 
Figure 25: Distribution of the total amounts of GCF TNF- $\alpha$ of the diseased and healthy sites in the strictly diagnosed periodontitis and healthy patients. Dark lines show medians.

\section{TNF- $\alpha$}

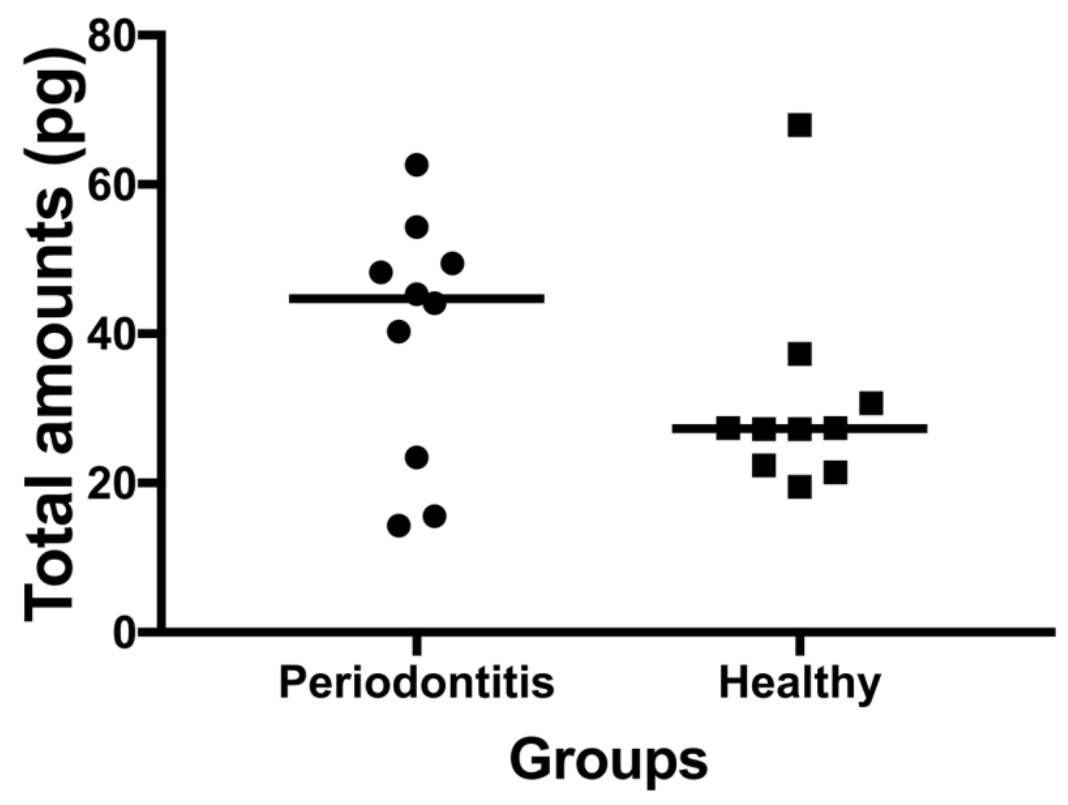

Mann-Whitney $U$ test was used to compare the total amounts of TNF- $\alpha$ between chronic periodontitis $(n=10)$ and periodontally healthy $(n=10)$ sites in strictly diagnosed chronic periodontitis and healthy patients, respectively. No significant differences were found between the two study subgroups $(p=0.226)$. 
Figure 26: ROC analysis of total amounts of GCF SOST to diagnose generalized chronic periodontitis.

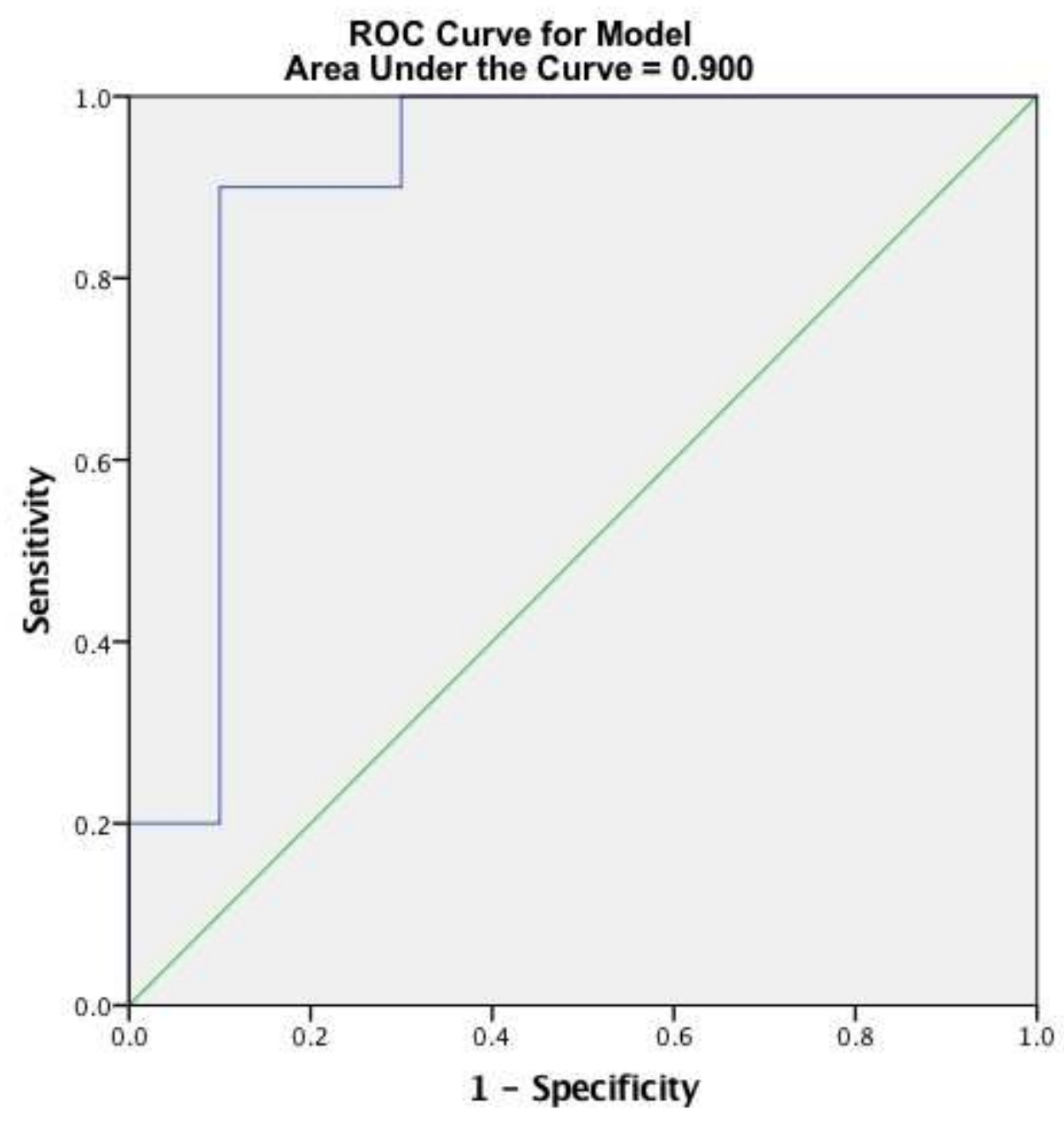


Figure 27: ROC analysis of total amounts of GCF Wnt-5a to diagnose generalized chronic periodontitis.

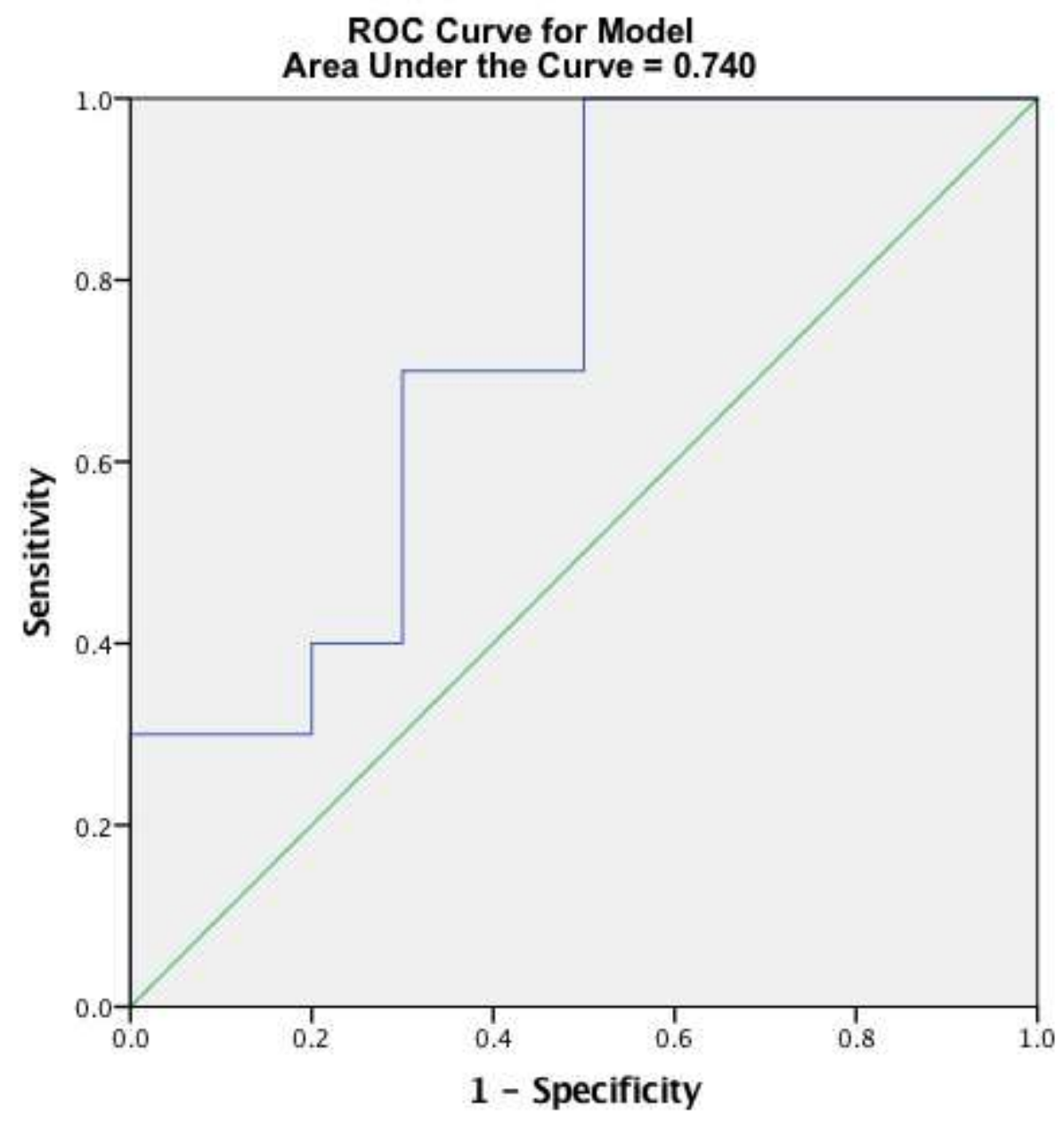


Figure 28: ROC analysis of total amounts of GCF TNF- $\alpha$ to diagnose generalized chronic periodontitis.

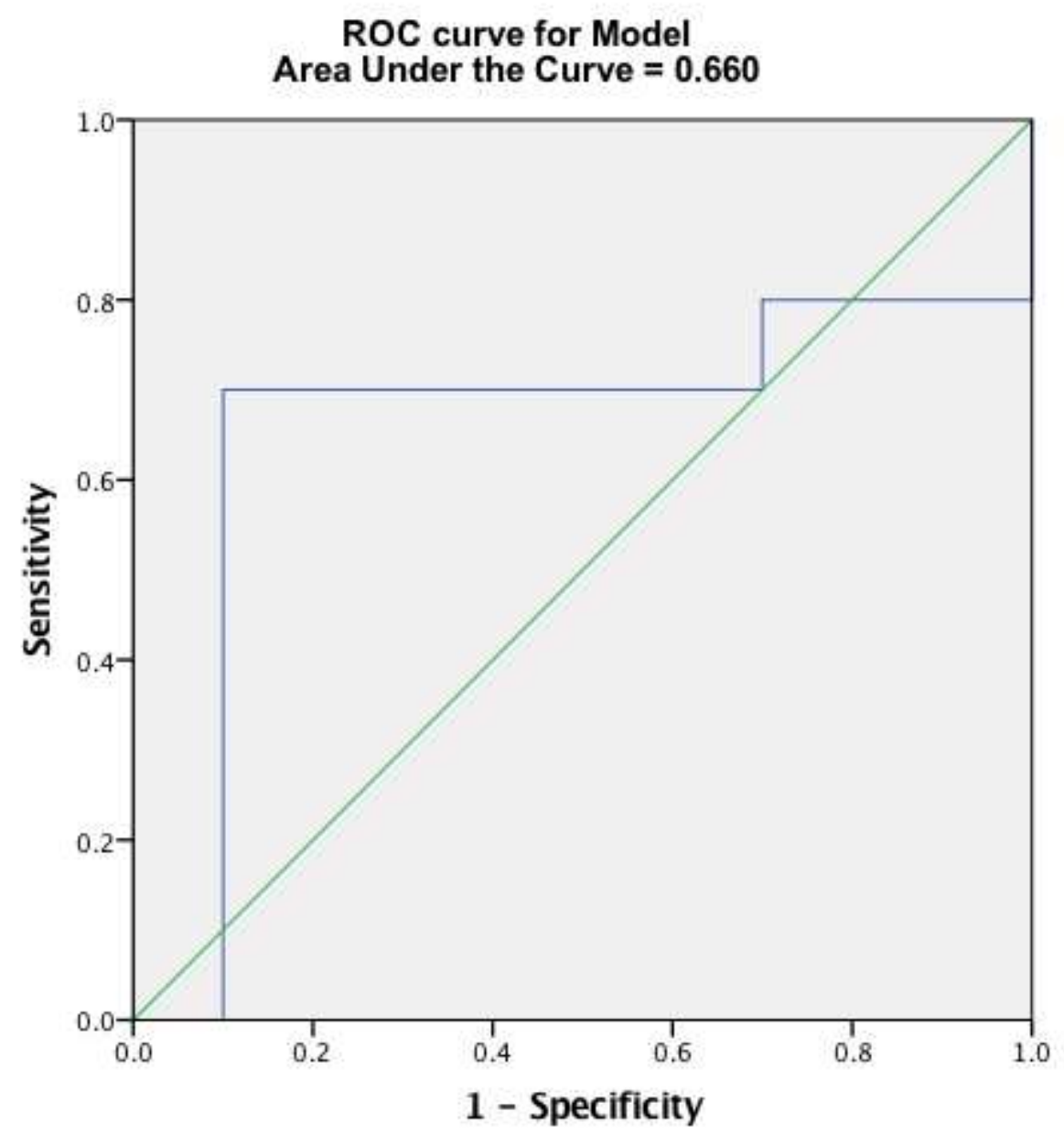




\section{BIBLIOGRAPHY}

Abbas F, Hart AA, Oosting J, van der Velden U. Effect of training and probing force on the reproducibility of pocket depth measurements. J Periodontal Res. 1982;17:226234.

Ahrens E. The crisis in clinical research: overcoming institutional obstacles. New York: Oxford University Press; 1992.

Al-Harthi LS, Cullinan MP, Leichter JW, Thomson WM. The impact of periodontitis on oral health-related quality of life: a review of the evidence from observational studies. Aust Dent J. 2013;58:274-277.

Albandar JM, Rams TE. Global epidemiology of periodontal diseases. Periodontol 2000. 2002;97:7-10

Alfano MC, Brownstein CN, Chasens AI, Kaslick RS. Passively generated increase in gingival crevicular fluid flow from human gingiva. J Dent Res. 1976;55:1132.

Alfano MC. The origin of gingival fluid. J Theor Biol. 1974;47:127-136.

Algate K, Haynes DR, Bartold PM, Crotti TN, Cantley MD. The effects of tumour necrosis factor-alpha on bone cells involved in periodontal alveolar bone loss; osteoclasts, osteoblasts and osteocytes. J Periodontal Res. 2016;51:549-566.

Almeida M, Han L, Bellido T, Manolagas SC, Kousteni S. Wnt proteins prevent apoptosis of both uncommitted osteoblast progenitors and differentiated osteoblasts by beta-catenin-dependent and -independent signaling cascades involving Src/ERK and phosphatidylinositol 3-kinase/AKT. J Biol Chem. 2005;280:41342-41351. 
American Dental Association. Council of Scientific Affairs. US Department of Health and Human Services. Public Health Service. Food Drug Administration. Revised 2012. http://www.ada.org/ /media/ADA/Member\%20Center/Flles/Dental_Radiographic_Ex aminations_2012.pdf [Accessed on February 10, 2018]

Armitage GC. Analysis of gingival crevice fluid and risk of progression of periodontitis. Periodontol 2000. 2004;34:109-119.

Armitage GC. Clinical evaluation of periodontal diseases. Periodontol 2000. 1995:7:39-53.

Armitage GC. Development of classification system for periodontal diseases and conditions. Ann Periodontol. 1999;4:1-6.

Arun KV, Talwar A, Kumar TS. T-helper cells in the etiopathogenesis of periodontal disease: A mini review. J Indian Soc Periodontol. 2011;15:4-10.

Assuma R, Oates T, Cochran D, Amar S, Graves DT. IL-1 and TNF antagonists inhibit the inflammatory response and bone loss in experimental periodontitis. J Immunol. 1998;160:403-409.

Babij P, Zhao W, Small C, Kharode Y, Yaworsky PJ, Bouxsein ML, Reddy PS, Bodine PV, Robinson JA, Bhat B, Marzolf J, Moran RA, Bex F. High bone mass in mice expressing a mutant LRP5 gene. J Bone Miner Res. 2003;18:960-974.

Baek K, Hwang HR, Park HJ, Kwon A, Qadir AS, Ko SH, Woo KM, Ryoo HM, Kim GS, Baek JH. TNF-a upregulates sclerostinexpression in obese mice fed a high-fat diet. Cell Physiol.2014;229:640-650.

Balemans W, Patel N, Ebeling M, Van Hul E, Wuyts W, Lacza C, Dioszegi M, Dikkers FG, Hildering P, Willems PJ, Verheij JB, Lindpaintner K, Vickery B, Foernzler D, Van 
Hul W. Identification of a $52 \mathrm{~kb}$ deletion downstream of the SOST gene in patients with van Buchem disease. J Med Genet. 2002;39 2:91-97.

Balemans W, Ebeling M, Patel N, Van Hul E, Olson P, Dioszegi M, Lacza C, Wuyts W, Van Den Ende J, Willems P, Paes-Alves AF, Hill S, Bueno M, Ramos FJ, Tacconi P, Dikkers FG, Stratakis C, Lindpaintner K, Vickery B, Foernzler D, Van Hul W. Increased bone density in sclerosteosis is due to the deficiency of a novel secreted protein (SOST). Hum Mol Genet. 2001;10:537-543.

Bandyopadhyay D, Marlow NM, Fernandes JK, Leite RS. Periodontal disease progression and glycaemic control among Gullah African Americans with type-2 diabetes. J Clin Periodontol. 2010;37:501-509.

Barbour SE, Nakashima K, Zhang JB, Tangada S, Hahn CL, Schenkein HA, Tew JG. Tobacco and smoking: environmental factors that modify the host response (immune system) and have an impact on periodontal health. Crit Rev Oral Biol Med. 1997;8:437460.

Baron R, Kneissel M. WNT signaling in bone homeostasis and disease: from human mutations to treatments. Nat Med. 2013;19:179-192.

Becerikli M, Jaurich H, Schira J, Schulte M, Döbele C, Wallner C, Abraham S, Wagner JM, Dadras M, Kneser U, Lehnhardt M, Behr B. Age-dependent alterations in osteoblast and osteoclast activity in human cancellous bone. J Cell Mol Med. 2017;21:2773-2781.

Belibasakis GN, Bostanci N. The RANKL-OPG system in clinical periodontology. J Clin Periodontol. 2012;39:239-248. 
Bergenfelz C, Medrek C, Ekström E, Jirström K, Janols H, Wullt M, Bredberg A, Leandersson K. Wnt-5a induces a tolerogenic phenotype of macrophages in sepsis and breast cancer patients. J Immunol. 2012;188:5448-5458.

Bergström J, Eliasson S, Dock J. A 10-year prospective study of tobacco smoking and periodontal health. J Periodontol. 2000;71:1338-1347.

Bergström J. Cigarette smoking as risk factor in chronic periodontal disease. Community Dent Oral Epidemiol. 1989;17:245-247.

Bergström J. Tobacco smoking and risk for periodontal disease. J Clin Periodontol. 2003;30:107-113.

Best AM, Burmeister JA, Gunsolley JC, Brooks CN, Schenkein HA. Reliability of attachment loss measurements in a longitudinal clinical trial. J Clin Periodontol. 1990;17:564-569.

Bhatt PM, Malgor R. Wnt5a: a player in the pathogenesis of atherosclerosis and other inflammatory disorders. Atherosclerosis. 2014;237:155-62.

Blumenthal A, Ehlers S, Lauber J, Buer J, Lange C, Goldmann T, Heine H, Brandt E, Reiling $\mathrm{N}$. The wingless homolog WNT5a and its receptor Frizzled-5 regulate inflammatory responses of human mononuclear cells induced by microbial stimulation. Blood. 2006;108:965-973.

Bostanci N, Ilgenli T, Emingil G, Afacan B, Han B, Töz H, Berdeli A, Atilla G, McKay IJ, Hughes FJ, Belibasakis GN. Differential expression of receptor activator of nuclear factor-kappaB ligand and osteoprotegerin mRNA in periodontal diseases. $J$ Periodontal Res. 2007;42:287-293. 
Bougault C, Briolay A, Boutet MA, Pilet P, Delplace S, Le Goff B, Guicheux J, Blanchard F, Magne D. Wnt5a is expressed in spondyloarthritis and exerts opposite effects on enthesis and bone in murine organ and cell cultures. Transl Res. 2015;166:627-638.

Bowers MR, Fisher LW, Termine JD, Somerman MJ. Connective tissue-associated proteins in crevicular fluid: potential markers for periodontal diseases. J Periodontol. 1989;60:448-451.

Boyden LM, Mao J, Belsky J, Mitzner L, Farhi A, Mitnick MA, Wu D, Insogna K, Lifton RP. High bone density due to a mutation in LDL-receptor-related protein 5 . N Engl $\mathrm{J}$ Med. 2002;346:1513-1521.

Brill N. The gingival pocket fluid. Studies of its occurrence, composition, and effect. Acta Odontol Scand. 1962:20:14.

Brown LJ, Loe H. Prevalence, extent, severity and progression of periodontal disease. Periodontol 2000. 1993:2:57-71.

Brownlee M. The pathobiology of diabetic complications: a unifying mechanism. Diabetes. 2005;54:1615-1625.

Brunkow ME, Gardner JC, Van Ness J, Paeper BW, Kovacevich BR, Proll S, Skonier JE, Zhao L, Sabo PJ, Fu Y, Alisch RS, Gillett L, Colbert T, Tacconi P, Galas D, Hamersma $\mathrm{H}$, Beighton $\mathrm{P}$, Mulligan J. Bone dysplasia sclerosteosis results from loss of the SOST gene product, a novel cystine knot-containing protein. Am J Hum Genet. 2001;68:577-589.

Buckwalter JA, Glimcher MJ, Cooper RR, Recker R. Bone biology. I: Structure, blood supply, cells, matrix, and mineralization. Instr Course Lect. 1996;45:371-86. 
Buduneli N, Kinane DF. Host-derived diagnostic markers related to soft tissue destruction and bone degradation in periodontitis. J Clin Periodontol. 2011;38(Suppl 11):85-105.

Campbell L, Millhouse E, Malcolm J, Culshaw S. T cells, teeth and tissue destruction - what do T cells do in periodontal disease? Mol Oral Microbiol. 2016;31:445-456.

Cao X, Chen D. The BMP signaling and in vivo bone formation. Gene. 2005;357:1-8.

Cekici A, Kantarci A, Hasturk H, Van Dyke TE. Inflammatory and immune pathways in the pathogenesis of periodontal disease. Periodontology 2000. 2014;64:57-80.

Chamoux E, Couture J, Bisson M, Morissette J, Brown JP, Roux S. The p62 P392L mutation linked to Paget's disease induces activation of human osteoclasts. Mol Endocrinol. 2009;23:1668-1680.

Champagne CM, Buchanan W, Reddy MS, Preisser JS, Beck JD, Offenbacher S. Potential for gingival crevice fluid measures as predictors of risk for periodontal diseases. Periodontol 2000. 2003;31:167-80.

Chapple IL, Cross IA, Glenwright HD, Matthews JB. Calibration and reliability of the Periotron 6000 for individual gingival crevicular fluid samples. J Periodontal Res. 1995;30:73-79.

Chávarry NG, Vettore MV, Sansone C, Sheiham A. The relationship between diabetes mellitus and destructive periodontal disease: a meta-analysis. Oral Health Prev Dent. 2009;7:107-127.

Cheng WC, Hughes FJ, Taams LS. The presence, function and regulation of IL-17 and Th17 cells in periodontitis. J Clin Periodontol. 2014;41:541-549. 
Chiquet BT, Blanton SH, Burt A, Ma D, Stal S, Mulliken JB, Hecht JT. Variation in WNT genes is associated with non-syndromic cleft lip with or without cleft palate. Hum Mol Genet. 2008;17:2212-2218.

Colić M, Gazivoda D, Vucević D, Vasilijić S, Rudolf R, Lukić A. Proinflammatory and immunoregulatory mechanisms in periapical lesions. Mol Immunol. 2009;47:101-113.

Crockett JC, Mellis DJ, Scott DI, Helfrich MH. New knowledge on critical osteoclast formation and activation pathways from study of rare genetic diseases of osteoclasts: focus on the RANK/RANKL axis. Osteoporos Int. 2011;22:1-20.

Crotti T, Smith MD, Hirsch R, Soukoulis S, Weedon H, Capone M, Ahern MJ, Haynes D. Receptor activator NF kappaB ligand (RANKL) and osteoprotegerin (OPG) protein expression in periodontitis. J Periodontal Res. 2003;38:380-387.

Cui Y, Niziolek PJ, MacDonald BT, Zylstra CR, Alenina N, Robinson DR, Zhong Z, Matthes S, Jacobsen CM, Conlon RA, Brommage R, Liu Q, Mseeh F, Powell DR, Yang QM, Zambrowicz B, Gerrits H, Gossen JA, He X, Bader M, Williams BO, Warman ML, Robling AG. Lrp5 functions in bone to regulate bone mass. Nat Med. 2011;17:684691.

Dallas SL, Prideaux M, Bonewald LF. The osteocyte: an endocrine cell ... and more. Endocr Rev. 2013;34:658-690.

Dandona P, Aljada A, Bandyopadhyay A. Inflammation: the link between insulin resistance, obesity and diabetes. Trends Immunol. 2004;25:4-7.

Day TF, Guo X, Garrett-Beal L, Yang Y. Wnt/beta-catenin signaling in mesenchymal progenitors controls osteoblast and chondrocyte differentiation during vertebrate skeletogenesis. Dev Cell. 2005;8:739-750. 
Delgado-Calle J, Sato AY, Bellido T. Role and mechanism of action of sclerostin in bone. Bone. 2017;96:29-37.

Di Benedetto A, Gigante I, Colucci S, Grano M. Periodontal disease: linking the primary inflammation to bone loss. Clin Dev Immunol. 2013;2013:503754.

Diarra D, Stolina M, Polzer K, Zwerina J, Ominsky MS, Dwyer D, Korb A, Smolen J, Hoffmann M, Scheinecker C, van der Heide D, Landewe R, Lacey D, Richards WG, Schett G. Dickkopf-1 is a master regulator of joint remodeling. Nat Med. 2007;13:156163.

Divaris K, Monda KL, North KE, Olshan AF, Reynolds LM, Hsueh WC, Lange EM, Moss K, Barros SP, Weyant RJ, Liu Y, Newman AB, Beck JD, Offenbacher S. Exploring the genetic basis of chronic periodontitis: a genome-wide association study. Hum Mol Genet. 2013;22:2312-24.

Drummond S, Canavarro C, Perinetti G, Teles R, Capelli J Jr. The monitoring of gingival crevicular fluid volume during orthodontic treatment: a longitudinal randomized split-mouth study. Eur J Orthod. 2012;34:109-113.

Eberhard J, Grote K, Luchtefeld M, Heuer W, Schuett H, Divchev D, Scherer R, Schmitz-Streit R, Langfeldt D, Stumpp N, Staufenbiel I, Schieffer B, Stiesch M. Experimental gingivitis induces systemic inflammatory markers in young healthy individuals: a single-subject interventional study. PLoS One. 2013;8:e55265.

Ebersole JL, Cappelli D. Acute-phase reactants in infections and inflammatory diseases. Periodontol 2000. 2000;23:19-49.

Ebersole JL. Humoral immune responses in gingival crevice fluid: local and systemic implications. Periodontol 2000. 2003;31:135-166. 
Egelberg J, Attstrom R. Comparison between orifice and intracrevicular methods of sampling gingival fluid. J Periodontal Res. 1973;8:384-388.

Eley B, Cox S. Advances in periodontal diagnosis 1. Traditional clinical methods of diagnosis. Br Dent J. 1998;184:12-16.

Frank S, Hubner G, Breier G, Longaker MT, Greenhalgh DG, Werner S. Regulation of vascular endothelial growth factor expression in cultured keratinocytes. Implications for normal and impaired wound healing. J Biol Chem. 1995;270:12607-12613.

Fujita $\mathrm{Y}$, Ito $\mathrm{H}$, Sekino $\mathrm{S}$, Numabe $\mathrm{Y}$. Correlations between pentraxin 3 or cytokine levels in gingival crevicular fluid and clinical parameters of chronic periodontitis. Odontology. 2012;100:215-221.

Gaffen SL, Hajishengallis G. A new inflammatory cytokine on the block: re-thinking periodontal disease and the Th1/Th2 paradigm in the context of Th17 cells and IL-17. J Dent Res. 2008;87:817-828.

Gargiulo AW, Wentz FM, Orban B. Dimensions and relations of the dentogingival junctions in humans. J Periodontol. 1961;32:261-267

Garlet GP, Cardoso CR, Campanelli AP, Ferreira BR, Avila-Campos MJ, Cunha FQ, Silva JS. The dual role of p55 tumour necrosis factor-alpha receptor in Actinobacillus actinomycetemcomitans-induced experimental periodontitis: host protection and tissue destruction. Clin Exp Immunol. 2007;147:128-138.

Gaspersic R, Stiblar-Martincic D, Osredkar J, Skaleric U. Influence of subcutaneous administration of recombinant TNF-alpha on ligature-induced periodontitis in rats. $\mathrm{J}$ Periodontal Res. 2003;38:198-203. 
Gemmell E, Yamazaki K, Seymour GJ. The role of T cells in periodontal disease: homeostasis and autoimmunity. Periodontol 2000. 2007;43:14-40.

Genco RJ, Borgnakke WS. Risk factors for periodontal disease. Periodontol 2000. 2013;62:59-94.

Genco RJ. Current view of risk factors for periodontal diseases. J Periodontol. 1996;67(10 Suppl):1041-1049.

Glass DA, Karsenty G. Canonical Wnt signaling in osteoblasts is required for osteoclast differentiation. Ann N Y Acad Sci. 2006;1068:117-130.

Gokul K, Faizuddin M, Pradeep AR. Estimation of the level of tumor necrosis factor- $\alpha$ in gingival crevicular fluid and serum in periodontal health \& disease: A biochemical study. Indian J Dent Res. 2012;23:348-352.

Goldring SR, Purdue PE, Crotti TN, Shen Z, Flannery MR, Binder NB, Ross FP, McHugh KP. Bone remodelling in inflammatory arthritis. Ann Rheum Dis. 2013;72(Suppl 2):52-55.

Gong Y, Slee RB, Fukai N, Rawadi G, Roman-Roman S, Reginato AM, Wang H, Cundy T, Glorieux FH, Lev D, Zacharin M, Oexle K, Marcelino J, Suwairi W, Heeger S, Sabatakos G, Apte S, Adkins WN, Allgrove J, Arslan-Kirchner M, Batch JA, Beighton P, Black GC, Boles RG, Boon LM, Borrone C, Brunner HG, Carle GF, Dallapiccola B, De Paepe A, Floege B, Halfhide ML, Hall B, Hennekam RC, Hirose T, Jans A, Jüppner H, Kim CA, Keppler-Noreuil K, Kohlschuetter A, LaCombe D, Lambert M, Lemyre E, Letteboer T, Peltonen L, Ramesar RS, Romanengo M, Somer H, Steichen-Gersdorf E, Steinmann B, Sullivan B, Superti-Furga A, Swoboda W, van den Boogaard MJ, Van Hul W, Vikkula M, Votruba M, Zabel B, Garcia T, Baron R, Olsen BR, Warman ML; Osteoporosis-Pseudoglioma Syndrome Collaborative Group. LDL 
receptor-related protein 5 (LRP5) affects bone accrual and eye development. Cell. 2001;107:513-523.

Goodson JM. Clinical measurements of periodontitis. J Clin Periodontol. 1986;13:446460.

Goodson JM. Gingival crevice fluid flow. Periodontology 2000. 2003;31:43-54.

Gordon MD, Nusse R. Wnt signaling: multiple pathways, multiple receptors, and multiple transcription factors. J Biol Chem. 2006;281:22429-22433.

Gravallese EM, Manning C, Tsay A, Naito A, Pan C, Amento E, Goldring SR. Synovial tissue in rheumatoid arthritis is a source of osteoclast differentiation factor. Arthritis Rheum. 2000;43:250-258.

Graves DT, Oates T, Garlet GP. Review of osteoimmunology and the host response in endodontic and periodontal lesions. J Oral Microbiol. 2011;3.

Graves DT, Oskoui M, Volejnikova S, Naguib G, Cai S, Desta T, Kakouras A, Jiang Y. Tumor necrosis factor modulates fibroblast apoptosis, PMN recruitment, and osteoclast formation in response to P. gingivalis infection. J Dent Res. 2001;80:18751879

Griffiths GS, Wilton JM, Curtis MA. Contamination of human gingival crevicular fluid by plaque and saliva. Arch Oral Biol. 1992;37:559-564.

Griffiths GS. Formation, collection and significance of gingival crevice fluid. Periodontology 2000. 2003;31:32-42.

Grimes DA, Schulz KF. An overview of clinical research: the lay of the land. Lancet. 2002;359:57-61. 
Grossi SG, Genco RJ, Machtei EE, Ho AW, Koch G, Dunford R, Zambon JJ, Hausmann E. Assessment of risk for periodontal disease. II. Risk indicators for alveolar bone loss. J Periodontol. 1995;66:23-29.

Guentsch A, Kramesberger M, Sroka A, Pfister W, Potempa J, Eick S. Comparison of gingival crevicular fluid sampling methods in patients with severe chronic periodontitis. J Periodontol. 2011;82:1051-1060.

Hajishengallis G. Immunomicrobial pathogenesis of periodontitis: keystones, pathobionts, and host response. Trends Immunol. 2014;35:3-11.

Hancock EB, Cray RJ, O'Leary TJ. The relationship between gingival crevicular fluid and gingival inflammation. A clinical and histologic study. J Periodontol. 1979;50:1319.

Hans M, Hans VM. Toll-like receptors and their dual role in periodontitis: a review. J Oral Sci. 2011;53:263-271.

Hayashi C, Gudino CV, Gibson FC 3rd, Genco CA. Review: Pathogen-induced inflammation at sites distant from oral infection: bacterial persistence and induction of cell-specific innate immune inflammatory pathways. Mol Oral Microbiol. 2010;25:305316.

Haynes KR, Pettit AR, Duan R, Tseng HW, Glant TT, Brown MA, Thomas GP. Excessive bone formation in a mouse model of ankylosing spondylitis is associated with decreases in Wnt pathway inhibitors. Arthritis Res Ther. 2012;14:R253.

Haytac MC, Ozcelik O, Mariotti A. Periodontal disease in men. Periodontol 2000. 2013;61:252-265. 
He X, Saint-Jeannet JP, Wang Y, Nathans J, Dawid I, Varmus H. A member of the Frizzled protein family mediatingaxis induction by Wnt-5A. Science. 1997;275:16521654.

Heiland GR, Zwerina K, Baum W, Kireva T, Distler JH, Grisanti M, Asuncion F, Li X, Ominsky M, Richards W, Schett G, Zwerina J. Neutralisation of Dkk-1 protects from systemic bone loss during inflammation and reduces sclerostin expression. Ann Rheum Dis. 2010;69:2152-2159.

Hill TP, Später D, Taketo MM, Birchmeier W, Hartmann C. Canonical Wnt/beta-catenin signaling prevents osteoblasts from differentiating into chondrocytes. Dev Cell. 2005;8:727-38.

Hofbauer LC, Heufelder AE. Role of receptor activator of nuclear factor-kappaB ligand and osteoprotegerin in bone cell biology. J Mol Med (Berl). 2001;79:243-253.

Hofbauer LC, Heufelder AE. Role of receptor activator of nuclear factor-kappaB ligand and osteoprotegerin in bone cell biology. J Mol Med (Berl). 2001;79:243-253.

Hofmann S, Grasberger H, Jung P, Bidlingmaier M, Vlotides J, Janssen OE, Landgraf $R$. The tumour necrosis factor-alpha induced vascular permeability is associated with a reduction of VE-cadherin expression. Eur J Med Res. 2002;7:171-176.

Holtfreter B, Albandar JM, Dietrich T, Dye BA, Eaton KA, Eke PI, Papapanou PN, Kocher T; Joint EU/USA Periodontal Epidemiology Working Group. Standards for reporting chronic periodontitis prevalence and severity in epidemiologic studies: Proposed standards from the Joint EU/USA Periodontal Epidemiology Working Group. J Clin Periodontol. 2015;42:407-412.

Hu H, Hilton MJ, Tu X, Yu K, Ornitz DM, Long F. Sequential roles of Hedgehog and Wnt signaling in osteoblast development. Development. 2005;132:49-60. 
Hughes FJ, Turner W, Belibasakis G, Martuscelli G. Effects of growth factors and cytokines on osteoblast differentiation. Periodontol 2000. 2006;41:48-72.

Hugoson A, Laurell L, Lundgren D. Frequency distribution of individuals aged 20-70 years according to severity of periodontal disease experience in 1973 and 1983. J Clin Periodontol. 1992;19:227-232.

Hulka BS, Wilcosky T. Biological markers in epidemiologic research. Arch Environ Health. 1988;43:83-89.

Janssen PT, Faber JA. The effect of patient-associated factors on the reproducibility of the mouth bleeding score. J Periodontal Res. 1988;23:318-321.

Jekel JF. Epidemiology, biostatistics, and preventive medicine. 3rd ed. Philadelphia: Saunders Elsevier; 2007

Jenkins ZA, van Kogelenberg M, Morgan T, Jeffs A, Fukuzawa R, Pearl E, Thaller C, Hing AV, Porteous ME, Garcia-Miñaur S, Bohring A, Lacombe D, Stewart F, Fiskerstrand T, Bindoff L, Berland S, Adès LC, Tchan M, David A, Wilson LC, Hennekam RC, Donnai D, Mansour S, Cormier-Daire V, Robertson SP. Germline mutations in WTX cause a sclerosing skeletal dysplasia but do not predispose to tumorigenesis. Nat Genet. 2009;41:95-100.

Johnson GK, Hill M. Cigarette smoking and the periodontal patient. J Periodontol. 2004;75:196-209.

Johnson-Greene D, Dehring M, Adams KM, Miller T, Arora S, Beylin A, Brandon R. Accuracy of self-reported educational attainment among diverse patient populations: a preliminary investigation. Arch Clin Neuropsychol. 1997;12:635-643. 
Kanzaki H, Chiba M, Shimizu Y, Mitani H. Periodontal ligament cells under mechanical stress induce osteoclastogenesis by receptor activator of nuclear factor kappaB ligand up-regulation via prostaglandin E2 synthesis. J Bone Miner Res. 2002;17:210-220.

Kato M, Patel MS, Levasseur R, Lobov I, Chang BH, Glass DA 2nd, Hartmann C, Li L, Hwang TH, Brayton CF, Lang RA, Karsenty G, Chan L. Cbfa1-independent decrease in osteoblast proliferation, osteopenia, and persistent embryonic eye vascularization in mice deficient in Lrp5, a Wnt coreceptor. J Cell Biol. 2002;157:303314.

Kawai VK, Stein CM, Perrien DS, Griffin MR. Effects of anti-tumor necrosis factor a agents on bone. Curr Opin Rheumatol. 2012;24:576-585.

Kehoe R, Wu SY, Leske MC, Chylack LT Jr. Comparing self-reported and physicianreported medical history. Am J Epidemiol. 1994;139:813-818.

Khosla S, Oursler MJ, Monroe DG. Estrogen and the skeleton. Trends Endocrinol Metab. 2012;23:576-581.

Khosla S. Minireview: the OPG/RANKL/RANK system. Endocrinology. 2001;142:5050-5055.

Kikuchi A, Yamamoto H, Sato A. Selective activation mechanisms of Wnt signaling pathways. Trends Cell Biol. 2009;19:119-129.

Kinane DF, Chestnutt IG. Smoking and periodontal disease. Crit Rev Oral Biol Med. 2000;11:356-365.

Kinane DF, Demuth DR, Gorr SU, Hajishengallis GN, Martin MH. Human variability in innate immunity. Periodontol 2000. 2007;45:14-34. 
Kinane DF, Lappin DF. Clinical, pathological and immunological aspects of periodontal disease. Acta Odontol Scand. 2001;59:154-160.

Kinane DF, Preshaw PM, Loos BG; Working Group 2 of Seventh European Workshop on Periodontology. Host-response: understanding the cellular and molecular mechanisms of host-microbial interactions--consensus of the Seventh European Workshop on Periodontology. J Clin Periodontol. 2011;38(Suppl 11):44-48.

Klingberg E, Nurkkala M, Carlsten H, Forsblad-d'Elia H. Biomarkers of bone metabolism in ankylosing spondylitis in relation to osteoproliferation and osteoporosis. J Rheumatol. 2014;41:1349-1356.

Klinger G. Sulcus fluid flow rate in relation to hormonal influence. Stomatol DDR. 1982;32:53-55.

Kobayashi S, Takahashi HE, Ito A, Saito N, Nawata M, Horiuchi H, Ohta H, Ito A, lorio R, Yamamoto N, Takaoka K. Trabecular minimodeling in human iliac bone. Bone. 2003;32:163-169.

Kornman KS. Mapping the pathogenesis of periodontitis: a new look. J Periodontol. 2008;79:1560-1568.

Krauss JL, Potempa J, Lambris JD, Hajishengallis G. Complementary Tolls in the periodontium: how periodontal bacteria modify complement and Toll-like receptor responses to prevail in the host. Periodontol 2000. 2010;52:141-162.

Krishnan V, Bryant HU, Macdougald OA. Regulation of bone mass by Wnt signaling. J Clin Invest. 2006;116:1202-1209.

Kühl M, Sheldahl LC, Park M, Miller JR, Moon RT. The Wnt/Ca2+ pathway: a new vertebrate Wnt signaling pathway takes shape. Trends Genet. 2000;16:279-283. 
Kumar PS, Matthews CR, Joshi V, de Jager M, Aspiras M. Tobacco smoking affects bacterial acquisition and colonization in oral biofilms. Infect Immun. 2011;79:47304738.

Laine ML, Crielaard W, Loos BG. Genetic susceptibility to periodontitis. Periodontol 2000. 2012;58:37-68.

Lammi L, Arte S, Somer M, Jarvinen H, Lahermo P, Thesleff I, Pirinen S, Nieminen P. Mutations in AXIN2 Cause Familial Tooth Agenesis and Predispose to Colorectal Cancer. Am J Hum Genet. 2004;74:1043-1050.

Lamster IB, Ahlo JK. Analysis of gingival crevicular fluid as applied to the diagnosis of oral and systemic diseases. Ann N Y Acad Sci. 2007;1098:216-229.

Lamster IB, Harper D, Goldstein S, Celenti RS, Oshrain RL. The effect of sequential sampling on crevicular fluid volume and enzyme activity. J Clin Periodontol. 1989;16:252-258.

Lamster IB, Hartley LJ, Vogel RI. Development of a biochemical profile for gingival crevicular fluid. Methodological considerations and evaluation of collagen-degrading and ground substance-degrading enzyme activity during experimental gingivitis. J Periodontol. 1985;56(11 Suppl):13-21.

Lamster IB, Oshrain RL, Gordon JM. Enzyme activity in human gingival crevicular fluid: considerations in data reporting based on analysis of individual crevicular sites. J Clin Periodontol. 1986;13:799-804.

Lamster IB. Evaluation of components of gingival crevicular fluid as diagnostic tests. Ann Periodontol. 1997;2:123-137. 
Lee HL, Yi T, Baek K, Kwon A, Hwang HR, Qadir AS, Park HJ, Woo KM, Ryoo HM, Kim GS, Baek JH. Tumor necrosis factor-alpha enhances the transcription of smad ubiquitination regulatory factor 1 in an activating protein-1- and Runx2-dependent manner. J Cell Physiol. 2013;228:1076-1086.

Lennon MA, Davies RM. Prevalence and distribution of alveolar bone loss in a population of 15-year-old school children. J Clin Periodontol. 1974;1:175-182.

Leppilahti JM, Hernández-Ríos PA, Gamonal JA, Tervahartiala T, BrignardelloPetersen R, Mantyla $P$, Sorsa T, Hernández M. Matrix metalloproteinases and myeloperoxidase in gingival crevicular fluid provide site-specific diagnostic value for chronic periodontitis. J Clin Periodontol. 2014;41:348-356.

Leris A, Roberts T, Jiang W, Newbold R, Mokbel K. WNT5A expression in human breast cancer. Anticancer Res. 2005;25:731-734.

Lerner UH. Inflammation-induced bone remodeling in periodontal disease and the influence of post-menopausal osteoporosis. J Dent Res. 2006;85:596-607.

Lerner UH. New molecules in the tumor necrosis factor ligand and receptor superfamilies with importance for physiological and pathological bone resorption. Crit Rev Oral Biol Med. 2004;15:64-81.

Li X, Warmington KS, Niu QT, Asuncion FJ, Barrero M, Grisanti M, Dwyer D, Stouch B, Thway TM, Stolina M, Ominsky MS, Kostenuik PJ, Simonet WS, Paszty C, Ke HZ. Inhibition of sclerostin by monoclonal antibody increases bone formation, bone mass, and bone strength in aged male rats. J Bone Miner Res. 2010;25:2647-2656.

Li X, Zhang Y, Kang H, Liu W, Liu P, Zhang J, Harris SE, Wu D. Sclerostin binds to LRP5/6 and antagonizes canonical Wnt signaling. J Biol Chem. 2005;280:1988319887. 
Li X, Ominsky MS, Warmington KS, Morony S, Gong J, Cao J, Gao Y, Shalhoub V, Tipton B, Haldankar R, Chen Q, Winters A, Boone T, Geng Z, Niu QT, Ke HZ, Kostenuik PJ, Simonet WS, Lacey DL, Paszty C. Sclerostin antibody treatment increases bone formation, bone mass, and bone strength in a rat model of postmenopausal osteoporosis. J Bone Miner Res. 2009;24:578-588.

Listgarten MA. Periodontal probing: what does it mean? J Clin Periodontol. 1980;7:165-176.

Liu D, Xu JK, Figliomeni L, Huang L, Pavlos NJ, Rogers M, Tan A, Price P, Zheng MH. Expression of RANKL and OPG mRNA in periodontal disease: possible involvement in bone destruction. Int J Mol Med. 2003;11:17-21.

Liu Y, Rubin B, Bodine PV, Billiard J.Wnt5a induces homodimerization and activation of Ror2 receptor tyrosine kinase. J Cell Biochem. 2008;105:497-502.

Löe H, Anerud A, Boysen H, Morrison E. Natural history of periodontal disease in man. Rapid, moderate and no loss of attachment in Sri Lankan laborers 14 to 46 years of age. J Clin Periodontol. 1986;13:431-445.

Löe $H$, Theilade E, Jensen SB. Experimental ginigivitis in man. J Periodontol. 1965;36:177-185.

Logan CY, Nusse R. The Wnt signaling pathway in development and disease. Annu Rev Cell Dev Biol. 2004;20:781-810.

Loos BG. Systemic markers of inflammation in periodontitis. J Periodontol. 2005;76:2106-2115. 
Maeda K, Kobayashi Y, Udagawa N, Uehara S, Ishihara A, Mizoguchi T, Kikuchi Y, Takada I, Kato S, Kani S, Nishita M, Marumo K, Martin TJ, Minami Y, Takahashi N. Wnt5a-Ror2 signaling between osteoblast-lineage cells and osteoclast precursors enhances osteoclastogenesis. Nat Med. 2012;18:405-412.

Maekawa T, Kulwattanaporn P, Hosur K, Domon H, Oda M, Terao Y, Maeda T, Hajishengallis G. Differential Expression and Roles of Secreted Frizzled-Related Protein 5 and the Wingless Homolog Wnt5a in Periodontitis. J Dent Res. 2017;96:571577.

Mahanonda R, Pichyangkul S. Toll-like receptors and their role in periodontal health and disease. Periodontol 2000. 2007;43:41-55.

Manau C, Echeverria A, Agueda A, Guerrero A, Echeverria JJ. Periodontal disease definition may determine the association between periodontitis and pregnancy outcomes. J Clin Periodontol. 2008;35:385-397.

Mann CJ. Observational research methods. Research design II: cohort, cross sectional, and case-control studies. Emerg Med J. 2003;20:54-60.

Martin LM, Leff M, Calonge N, Garrett C, Nelson DE. Validation of self-reported chronic conditions and health services in a managed care population. Am J Prev Med 2000;18:215-218.

Michalowicz BS, Diehl SR, Gunsolley JC, Sparks BS, Brooks CN, Koertge TE, Califano JV, Burmeister JA, Schenkein HA. Evidence of a substantial genetic basis for risk of adult periodontitis. J Periodontol. 2000;71:1699-1707.

Mikels AJ, Nusse R. Purified Wnt5a protein activates or inhibits b-catenin-TCF signaling depending on receptor context. PLoS Biol. 2006;4:e115. 
Mogi M, Otogoto J, Ota N, Togari A. Differential expression of RANKL and osteoprotegerin in gingival crevicular fluid of patients with periodontitis. J Dent Res. 2004;83:166-169.

Mol A. Imaging methods in periodontology. Periodontol 2000. 2004;34:34-48.

Murphy KM, Reiner SL. The lineage decisions of helper T cells. Nat Rev Immunol. 2002;2:933-944.

Nanbara H, Wara-aswapati N, Nagasawa T, Yoshida $\mathrm{Y}$, Yashiro R, Bando $\mathrm{Y}$, Kobayashi H, Khongcharoensuk J, Hormdee D, Pitiphat W, Boch JA, Izumi Y. Modulation of Wnt5a expression by periodontopathic bacteria. PLoS One. 2012;7:e34434.

Natarajan S, Lipsitz SR, Nietert PJ. Self-report of high cholesterol: determinants of validity in U.S. adults. Am J Prev Med. 2002;23:13-21.

Newell SA, Girgis A, Sanson-Fisher RW, Savolainen NJ. The accuracy of self-reported health behaviors and risk factors relating to cancer and cardiovascular disease in the general population: a critical review. Am J Prev Med 1999;17:211-229.

Nibali L, Di lorio A, Tu YK, Vieira AR. Host genetics role in the pathogenesis of periodontal disease and caries. J Clin Periodontol. 2017;44 (Suppl 18):S52-S78.

Nordenram G, Davidson T, Gynther G, Helgesson G, Hultin M, Jemt T, Lekholm U, Nilner K, Norlund A, Rohlin M, Sunnegårdh-Grönberg K, Tranæus S. Qualitative studies of patients' perceptions of loss of teeth, the edentulous state and prosthetic rehabilitation: a systematic review with meta-synthesis. Acta Odontol Scand. 2013;71:937-951. 
Oderup C, LaJevic M, Butcher EC. Canonical and noncanonical Wnt proteins program dendritic cell responses for tolerance. J Immunol. 2013;190:6126-6134.

Okura Y, Urban LH, Mahoney DW, Jacobsen SJ, Rodeheffer RJ. Agreement between self-report questionnaires and medical record data was substantial for diabetes, hypertension, myocardial infarction and stroke but not for heart failure. J Clin Epidemiol. 2004;57:1096-1103.

Ominsky MS, Boyce RW, Li X, Ke HZ. Effects of sclerostin antibodies in animal models of osteoporosis. Bone. 2017;96:63-75.

Osta B, Benedetti G, Miossec P. Classical and paradoxical effects of TNF-alpha on bone homeostasis. Front Immunol. 2014;5:48.

Ozkavaf A, Aras H, Huri CB, Mottaghian-Dini F, Tözüm TF, Etikan I, Yamalik N, Caglayan $\mathrm{F}$. Relationship between the quantity of gingival crevicular fluid and clinical periodontal status. J Oral Sci. 2000;42:231-238.

Page RC, Eke PI. Case definitions for use in population-based surveillance of periodontitis. J Periodontol. 2007;78(7 Suppl):1387-1399.

Page RC, Schroeder HE. Pathogenesis of inflammatory periodontal disease. A summary of current work. Lab Invest. 1976;34:235-249.

Page RC. The role of inflammatory mediators in the pathogenesis of periodontal disease. J Periodont Res. 1991;26:230-242.

Papapanou PN, Wennstrom JL, Grondahl K. A 10-year retrospective study of periodontal disease progression. J Clin Periodontol. 1989:16:403-411. 
Papapanou PN, Wennström JL. Radiographic and clinical assessments of destructive periodontal disease. J Clin Periodontol. 1989;16:609-612.

Papapanou PN. Periodontal diseases: epidemiology. Ann Periodontol. 1996;1:1-36.

Pereira C, Schaer DJ, Bachli EB, Kurrer MO, Schoedon G. Wnt5A/CaMKII signaling contributes to the inflammatory response of macrophages and is a target for the antiinflammatory action of activated protein $\mathrm{C}$ and interleukin-10. Arterioscler Thromb Vasc Biol. 2008;28:504-510.

Persson GR, Page RC. Effect of sampling time and repetition on gingival crevicular fluid and aspartate aminotransferase activity. J Periodontal Res. 1990;25:236-242.

Phan TC, Xu J, Zheng MH. Interaction between osteoblast and osteoclast: impact in bone disease. Histol Histopathol. 2004;19:1325-1344.

Pihlstrom BL. Periodontal risk assessment, diagnosis and treatment planning. Periodontol 2000. 2001;25:37-58.

Polson AM. The research team, calibration, and quality assurance in clinical trials in periodontics. Ann Periodontol. 1997;2:75-82.

Poole KE, van Bezooijen RL, Loveridge N, Hamersma H, Papapoulos SE, Löwik CW, Reeve J. Sclerostin is a delayed secreted product of osteocytes that inhibits bone formation. FASEB J. 2005;19:1842-1844.

Preshaw PM, Seymour RA, Heasman PA. Current concepts in periodontal pathogenesis. Dent Update. 2004;31:570-578.

Qian D, Jones C, Rzadzinska A, Mark S, Zhang X, Steel KP, Dai X, Chen P. Wnt5a functions in planar cell polarity regulation in mice. Dev Biol. 2007;306:121-133. 
Rakic M, Struillou X, Petkovic-Curcin A, Matic S, Canullo L, Sanz M, Vojvodic D. Estimation of bone loss biomarkers as a diagnostic tool for peri-implantitis. J Periodontol. 2014;85:1566-1574.

Rathnayake N, Akerman S, Klinge B, Lundegren N, Jansson H, Tryselius Y, Sorsa T, Gustafsson A. Salivary biomarkers for detection of systemic diseases. PLoS One. 2013;8:e61356.

Rauner M, Sipos W, Pietschmann P. Age-dependent Wnt gene expression in bone and during the course of osteoblast differentiation. Age (Dordr). 2008;30:273-282.

Reis C, DA Costa AV, Guimarães JT, Tuna D, Braga AC, Pacheco JJ, Arosa FA, Salazar F, Cardoso EM. Clinical improvement following therapy for periodontitis: Association with a decrease in IL-1 and IL-6. Exp Ther Med. 2014;8:323-327.

Robinson JR, Young TK, Roos LL, Gelskey DE. Estimating the burden of disease. Comparing administrative data and self-reports. Med Care. 1997;35:932-947.

Rodda SJ, McMahon AP. Distinct roles for Hedgehog and canonical Wnt signaling in specification, differentiation and maintenance of osteoblast progenitors. Development. 2006;133:3231-3244.

Rogers JE, Li F, Coatney DD, Rossa C, Bronson P, Krieder JM, Giannobile WV, Kirkwood KL. Actinobacillus actinomycetemcomitans lipopolysaccharide-mediated experimental bone loss model for aggressive periodontitis. J Periodontol. 2007;78:550-558.

Rossini M, Gatti D, Adami S. Involvement of WNT/ $\beta$-catenin signaling in the treatment of osteoporosis. Calcif Tissue Int. 2013;93:121-132. 
Rossomando EF, Kennedy JE, Hadjimichael J. Tumour necrosis factor alpha in gingival crevicular fluid as a possible indicator of periodontal disease in humans. Arch Oral Biol. 1990;35:431-434.

Saad CG, Ribeiro AC, Moraes JC, Takayama L, Goncalves CR, Rodrigues MB, de Oliveira RM, Silva CA, Bonfa E, Pereira RM. Low sclerostin levels: a predictive marker of persistent inflammation in ankylosing spondylitis during anti-tumor necrosis factor therapy? Arthritis Res Ther 2012;14:R216.

Sakata M, Shiba H, Komatsuzawa H, Fujita T, Ohta K, Sugai M, Suginaka H, Kurihara $\mathrm{H}$. Expression of osteoprotegerin (osteoclastogenesis inhibitory factor) in cultures of human dental mesenchymal cells and epithelial cells. J Bone Miner Res. 1999;14:1486-1492.

Salvi GE, Brown CE, Fujihashi K, Kiyono H, Smith FW, Beck JD, Offenbacher S. Inflammatory mediators of the terminal dentition in adult and early onset periodontitis. J Periodontal Res. 1998;33:212-225.

Sato A, Yamamoto H, Sakane H, Koyama H, Kikuchi A. Wnt5a regulates distinct signalling pathways by binding to Frizzled2. EMBO J. 2010;29:41-54.

Schenkein HA. Host responses in maintaining periodontal health and determining periodontal disease. Periodontol 2000. 2006;40:77-93.

Schett G. Joint remodelling in inflammatory disease. Ann Rheum Dis. 2007;66(Suppl 3):42-44.

Schulte DM, Müller N, Neumann K, Oberhäuser F, Faust M, Güdelhöfer H, Brandt B, Krone W, Laudes M. Pro-inflammatory wnt5a and anti-inflammatory sFRP5 are differentially regulated by nutritional factors in obese human subjects. PLoS One. 2012;7:e32437. 
Schulte PA. A conceptual framework for the validation and use of biologic markers. Environ Res. 1989;48:129-144.

Scott DA, Krauss J. Neutrophils in periodontal inflammation. Front Oral Biol. 2012;15:56-83.

Sen M, Chamorro M, Reifert J, Corr M, Carson DA. Blockade of Wnt-5A/frizzled 5 signaling inhibits rheumatoid synoviocyte activation. Arthritis Rheum. 2001;44:772-81.

Sen M, Lauterbach K, El-Gabalawy H, Firestein GS, Corr M, Carson DA. Expression and function of wingless and frizzled homologs in rheumatoid arthritis. Proc Natl Acad Sci U S A. 2000;97:2791-2796.

Seymour GJ, Greenspan JS. The phenotypic characterization of lymphocyte subpopulations in established human periodontal disease. J Periodontal Res. 1979;14:39-46.

Shen L, Zhou S, Glowacki J. Effects of age and gender on WNT gene expression in human bone marrow stromal cells. J Cell Biochem. 2009;106:337-343.

Silva TA, Garlet GP, Fukada SY, Silva JS, Cunha FQ. Chemokines in oral inflammatory diseases: Apical periodontitis and periodontal disease. J Dent Res. 2007;86:306-319.

Simonet WS, Lacey DL, Dunstan CR, Kelley M, Chang MS, Lüthy R, Nguyen HQ, Wooden S, Bennett L, Boone T, Shimamoto G, DeRose M, Elliott R, Colombero A, Tan HL, Trail G, Sullivan J, Davy E, Bucay N, Renshaw-Gegg L, Hughes TM, Hill D, Pattison W, Campbell P, Sander S, Van G, Tarpley J, Derby P, Lee R, Boyle WJ. Osteoprotegerin: a novel secreted protein involved in the regulation of bone density. Cell. 1997;89:309-319. 
Sims NA, Gooi JH. Bone remodeling: Multiple cellular interactions required for coupling of bone formation and resorption. Semin Cell Dev Biol. 2008;19:444-451.

Slade GD, Spencer AJ. Periodontal attachment loss among adults aged 60+ in South Australia. Community Dent Oral Epidemiol. 1995;23:237-242.

Smith QT, Au GS, Freese PL, Osborn JB, Stoltenberg JL. Five parameters of gingival crevicular fluid from eight surfaces in periodontal health and disease. J Periodontal Res. 1992;27:466-75.

Smith QT, Geegan SJ. Repeated measurement of crevicular fluid parameters at different sites. J Clin Periodontol. 1991;18:171-176.

Sobacchi C, Schulz A, Coxon FP, Villa A, Helfrich MH. Osteopetrosis: genetics, treatment and new insights into osteoclast function. Nat Rev Endocrinol. 2013;9:522536.

Spencer GJ, Utting JC, Etheridge SL, Arnett TR, Genever PG. Wnt signalling in osteoblasts regulates expression of the receptor activator of NFkappaB ligand and inhibits osteoclastogenesis in vitro. J Cell Sci. 2006;119:1283-1296.

Stashenko P, Jandinski JJ, Fujiyoshi P, Rynar J, Socransky SS. Tissue levels of bone resorptive cytokines in periodontal disease. J Periodontol. 1991;62:504-509.

Stephen LX, Hamersma H, Gardner J, Beighton P. Dental and oral manifestations of sclerosteosis. Int Dent J. 2001;51:287-290.

Stoller NH, Karras DC, Johnson LR. Reliability of crevicular fluid measurements taken in the presence of supragingival plaque. J Periodontol. 1990;61:670-673. 
Stoller NH, Karras DC, Johnson LR. Reliability of crevicular fluid measurements taken in the presence of supragingival plaque. J Periodontol. 1990;61:670-673.

Taba M Jr, Kinney J, Kim AS, Giannobile WV. Diagnostic biomarkers for oral and periodontal diseases. Dent Clin North Am. 2005;49:551-571.

Takayanagi $\mathrm{H}$, lizuka $\mathrm{H}$, Juji T, Nakagawa T, Yamamoto A, Miyazaki T, Koshihara $\mathrm{Y}$, Oda H, Nakamura K, Tanaka S. Involvement of receptor activator of nuclear factor kappaB ligand/osteoclast differentiation factor in osteoclastogenesis from synoviocytes in rheumatoid arthritis. Arthritis Rheum. 2000;43:259-269.

Taut AD, Jin Q, Chung JH, Galindo-Moreno P, Yi ES, Sugai JV, Ke HZ, Liu M, Giannobile WV. Sclerostin antibody stimulates bone regeneration after experimental periodontitis. J Bone Miner Res. 2013;28:2347-2356.

Taylor GW, Burt BA, Becker MP, Genco RJ, Shlossman M. Glycemic control and alveolar bone loss progression in type 2 diabetes. Ann Periodontol. 1998;3:30-39.

Teitelbaum SL, Ross FP. Genetic regulation of osteoclast development and function. Nat Rev Genet. 2003;4:638-649.

Teitelbaum SL. Bone resorption by osteoclasts. Science. 2000;289(5484):1504-1508.

Teitelbaum SL. Osteoclasts; culprits in inflammatory osteolysis. Arthritis Res Ther. 2006;8:201.

Teles RP, Likhari V, Socransky SS, Haffajee AD. Salivary cytokine levels in subjects with chronic periodontitis and in periodontally healthy individuals: A cross-sectional study. J Periodontal Res. 2009;44:411-417. 
Teng YT, Nguyen H, Gao X, Kong YY, Gorczynski RM, Singh B, Ellen RP, Penninger JM. Functional human T-cell immunity and osteoprotegerin ligand control alveolar bone destruction in periodontal infection. J Clin Invest. 2000;106:R59-67.

Tomar SL, Asma S. Smoking-attributable periodontitis in the United States: findings from NHANES III. National Health and Nutrition Examination Survey. J Periodontol. 2000;71:743-751.

Topol L, Jiang X, Choi H, Garrett-Beal L, Carolan PJ, Yang Y. Wnt-5a inhibits the canonical Wnt pathway by promoting GSK-3-independent beta-catenin degradation. J Cell Biol. 2003;162:899-908.

Torrungruang K, Nisapakultorn K, Sutdhibhisal S, Tamsailom S, Rojanasomsith K, Vanichjakvong O, Prapakamol S, Premsirinirund T, Pusiri T, Jaratkulangkoon O, Kusump S, Rajatanavin R. The effect of cigarette smoking on the severity of periodontal disease among older Thai adults. J Periodontol. 2005;76:566-572.

Tözüm TF, Hatipoğlu H, Yamalik N, Gürsel M, Alptekin NO, Ataoğlu T, Marakoğlu I, Gürsoy UK, Eratalay K. Critical steps in electronic volume quantification of gingival crevicular fluid: the potential impact of evaporation, fluid retention, local conditions and repeated measurements. J Periodontal Res. 2004;39:344-357.

Uitto VJ, Overall CM, McCulloch C. Proteolytic host cell enzymes in gingival crevice fluid. Periodontol 2000. 2003;31:77-104.

Ulker AE, Tulunoglu O, Ozmeric N, Can M, Demirtas S. The evaluation of cystatin C, IL-1beta, and TNF-alpha levels in total saliva and gingival crevicular fluid from 11- to 16-year-old children. J Periodontol. 2008;79:854-860.

Ustün K, Alptekin NO. The effect of tobacco smoking on gingival crevicular fluid volume. Eur J Dent. 2007;1:236-239. 
Vaithilingam RD, Safii SH, Baharuddin NA, Ng CC, Cheong SC, Bartold PM, Schaefer AS, Loos BG. Moving into a new era of periodontal genetic studies: relevance of large case-control samples using severe phenotypes for genome-wide association studies. J Periodont Res. 2014;49:683-695.

van Bezooijen RL, Bronckers AL, Gortzak RA, Hogendoorn PC, van der Wee-Pals L, Balemans W, Oostenbroek HJ, Van Hul W, Hamersma H, Dikkers FG, Hamdy NA, Papapoulos SE, Löwik CW. Sclerostin in mineralized matrices and van Buchem disease. J Dent Res. 2009;88:569-74.

van der Weijden GA, Hioe KP: A systematic review of the effectiveness of selfperformed mechanical plaque removal in adults with gingivitis using a manual toothbrush. J Clin Periodontol. 2005;32(Suppl 6):214-228.

Veeman MT, Axelrod JD, Moon RT. A second canon. Functions and mechanisms of beta-catenin-independent Wnt signaling. Dev Cell. 2003;5:367-377.

Wada K, Yatsuya H, Ouyang P, Otsuka R, Mitsuhashi H, Takefuji S, Matsushita K, Sugiura K, Hotta Y, Toyoshima H, Tamakoshi K. Self-reported medical history was generally accurate among Japanese workplace population. J Clin Epidemiol. 2009;62:306-313.

Wenzel A. Computer-aided image manipulation of intraoral radiographs to enhance diagnosis in dental practice: a review. Int Dent J. 1993;43:99-108.

White SC, Heslop EW, Hollender LG, Mosier KM, Ruprecht A, Shrout MK; American Academy of Oral and Maxillofacial Radiology, ad hoc Committee on Parameters of Care. Parameters of radiologic care: an official report of the American Academy of 
Oral and Maxillofacial Radiology. Oral Surg Oral Med Oral Pathol Oral Radiol Endod. 2001;91:498-511.

Wittkowske C, Reilly GC, Lacroix D, Perrault CM. In Vitro Bone Cell Models: Impact of Fluid Shear Stress on Bone Formation. Front Bioeng Biotechnol. 2016;4:87.

Wijenayaka AR, Kogawa M, Lim HP, Bonewald LF, Findlay DM, Atkins GJ. Sclerostin stimulates osteocyte support of osteoclast activity by a RANKL-dependent pathway. PLoS One. 2011;6:e25900.

Wilson C, Schulz S, Waldman SA. Biomarker development, commercialization, and regulation: individualization of medicine lost in translation. Clin Pharmacol Ther. 2007;81:153-155.

Wong BKJ, Leichter JW, Chandler NP, Cullinan MP, Holborow DW. Radiographic study of ethnic variation in alveolar bone height among New Zealand dental students. J Periodontol. 2007;78:1070-1074

Xu LY, Ma N, Qiu LH, Yu YQ, Zhong M, Yu JT, Xue M. Expression of Wnt5a in chronic apical periodontitis and its clinical significance. Shanghai Kou Qiang Yi Xue. 2015;24:470-474.

Yao Z, Li P, Zhang Q, Schwarz EM, Keng P, Arbini A, Boyce BF, Xing L. Tumor necrosis factor-alpha increases circulating osteoclast precursor numbers by promoting their proliferation and differentiation in the bone marrow through up-regulation of $\mathrm{c}$ Fms expression. J Biol Chem. 2006;281:11846-11855.

Yasuda H, Shima N, Nakagawa N, Yamaguchi K, Kinosaki M, Mochizuki S, Tomoyasu A, Yano K, Goto M, Murakami A, Tsuda E, Morinaga T, Higashio K, Udagawa N, Takahashi N, Suda T. Osteoclast differentiation factor is a ligand for 
osteoprotegerin/osteoclastogenesis-inhibitory factor and is identical to TRANCE/RANKL. Proc Natl Acad Sci U S A. 1998;95:3597-3602.

Yun TJ, Chaudhary PM, Shu GL, Frazer JK, Ewings MK, Schwartz SM, Pascual V, Hood LE, Clark EA. OPG/FDCR-1, a TNF receptor family member, is expressed in lymphoid cells and is up-regulated by ligating CD40. J Immunol. 1998;161:6113-6121.

Zhang Q, Guo R, Schwarz EM, Boyce BF, Xing L. TNF inhibits production of stromal cell-derived factor 1 by bone stromal cells and increases osteoclast precursor mobilization from bone marrow to peripheral blood. Arthritis Res Ther. 2008;10:R37.

Zhao L, Huang J, Zhang H, Wang Y, Matesic LE, Takahata M, Awad H, Chen D, Xing L. Tumor necrosis factor inhibits mesenchymal stem cell differentiation into osteoblasts via the ubiquitin E3 ligase Wwp1. Stem Cells. 2011;29:1601-1610.

Zhao Y, Zhang C, Huang Y, Yu Y, Li R, Li M, Liu N, Liu P, Qiao J. Up-regulated expression of WNT5a increases inflammation and oxidative stress via PI3K/AKT/NFKB signaling in the granulosa cells of PCOS patients. J Clin Endocrinol Metab. 2015;100:201-211. 